\title{
Benchmarks and Models for 1-D Radiation Transport in Stochastic Participating Media
}

\author{
D. S. Miller
}

Ph.D Dissertation

\section{August 2000}

U.S. Department of Energy

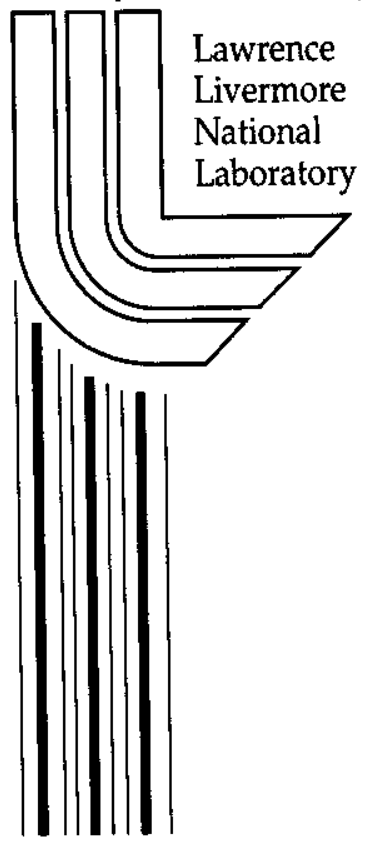




\section{DISCLAIMER}

This document was prepared as an account of work sponsored by an agency of the United States Government. Neither the United States Government nor the University of California nor any of their employees, makes any warranty, express or implied, or assumes any legal liability or responsibility for the accuracy, completeness, or usefulness of any information, apparatus, product, or process disclosed, or represents that its use would not infringe privately owned rights. Reference herein to any specific commercial product, process, or service by trade name, trademark, manufacturer, or otherwise, does not necessarily constitute or imply its endorsement, recommendation, or favoring by the United States Government or the University of California. The views and opinions of authors expressed herein do not necessarily state or reflect those of the United States Government or the University of California, and shall not be used for advertising or product endorsement purposes.

Work performed under the auspices of the U. S. Department of Energy by the University of California Lawrence Livermore National Laboratory under Contract W-7405-Eng-48.

This report has been reproduced directly from the best available copy.

Available to DOE and DOE contractors from the Office of Scientific and Technical Information P.O. Box 62, Oak Ridge, TN 37831

Prices available from (423) 576-8401 http://apollo.osti.gov/bridge/

Available to the public from the National Technical Information Service

U.S. Department of Commerce 5285 Port Royal Rd., Springfield, VA 22161 http://www.ntis.gov/

OR

Lawrence Livermore National Laboratory Technical Information Department's Digital Library http://www.llnl.gov/tid/Library.html 


\section{Benchmarks and Models for 1-D Radiation Transport in Stochastic Participating Media}

by

DAVID SCOTT MILLER

B.S. (California State University, San Francisco) 1991

M.S. (California State University, San Francisco) 1994

M.S. (University of California, Davis) 1996

\section{DISSERTATION}

Submitted in partial satisfaction of the requirements for the degree of

DOCTOR OF PHILOSOPHY

in

ENGINEERING-APPLIED SCIENCE

in the

OFFICE OF GRADUATE STUDIES

of the

UNIVERSITY OF CALIFORNIA

DAVIS

Approved:

Committee in Charge

2000 


\begin{abstract}
Benchmark calculations for radiation transport coupled to a material temperature equation in a 1-D slab and 1-D spherical geometry binary random media are presented. The mixing statistics are taken to be homogeneous Markov statistics in the 1-D slab but only approximately Markov statistics in the 1-D sphere. The material chunk sizes are described by Poisson distribution functions. The material opacities are first taken to be constant and then allowed to vary as a strong function of material temperature.
\end{abstract}

Benchmark values and variances for time evolution of the ensemble average of material temperature energy density and radiation transmission are computed via a Monte Carlo type method. These benchmarks are used as a basis for comparison with three other approximate methods of solution. One of these approximate methods is simple atomic mix. The second approximate model is an adaptation of what is commonly called the Levermore-Pomraning model and which is refered to here as the standard model. It is shown that recasting the temperature coupling as a type of effective scattering can be useful in formulating the third approximate model, an adaptation of a model due to Su and Pomraning which attempts to account for the effects of scattering in a stochastic context. This last adaptation shows consistent improvement over both the atomic mix and standard models when used in the 1-D slab geometry but shows limited improvement in the 1-D spherical geometry.

Benchmark values are also computed for radiation transmission from the 1-D sphere without material heating present. This is to evaluate the performance of the standard model on this geometry - something which has never been done before. 
All of the various tests demonstrate the importance of stochastic structure on the solution. Also demonstrated are the range of usefulness and limitations of a simple atomic mix formulation. 


\section{Acknowledgments}

There are number of people deserving of thanks and without whom this work would never have been accomplished on time.

The two main people are my advisors Frank Graziani and Garry Rodrigue. These two provided guidance, technical help, and all around good advise all along the way.

Niels Jensen not only stepped in at the last minute to serve as a committee member, he read the dissertation and got back to me with his feedback in record time.

Next are the folks from the transport community who gave of their time to help me understand the complexities of radiation transport: Marv Adams, Peter Brown, Britton Chang, Paul Nowak, Todd Palmer, Mike Zika, and any others I might have missed.

Archie, Donna Clifford, and Estelle Miller over at the Department of Applied Science office made all of the bureaucratic tortures endurable.

The Department of Energy and the Lawrence Livermore National Laboratory supported me throughout this process and provided me with the (bare minimum) recourses necessary to complete this work.

And last, but certainly not least, is my girlfriend Sarah. She tolerated my long work weeks, looked out for me and my home life, and provided unfailing encouragement during the stressful final stages of the writing.

Thanks to you all, it is done. 
Title Page..$\ldots \ldots \ldots \ldots \ldots \ldots \ldots \ldots \ldots \ldots \ldots \ldots \ldots \ldots \ldots$

Abstract $\ldots \ldots \ldots \ldots \ldots \ldots \ldots \ldots \ldots \ldots \ldots \ldots \ldots \ldots \ldots \ldots \ldots \ldots \ldots$, ii

Acknowledgments.$\ldots \ldots \ldots \ldots \ldots \ldots \ldots \ldots \ldots \ldots \ldots \ldots \ldots$ iv

INTRODUCTION AND SURVEY $\ldots \ldots \ldots \ldots \ldots \ldots \ldots \ldots \ldots \ldots . . \ldots \ldots$

1.0 Motivations For the Research $\ldots \ldots \ldots \ldots \ldots \ldots \ldots \ldots \ldots$

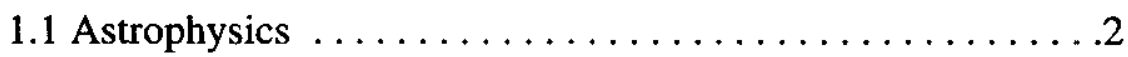

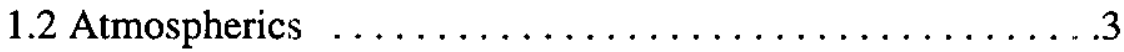

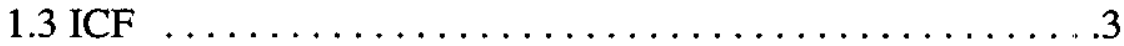

2.0 What is a Binary Stochastic Media? . ............... 4

2.1 Absolute Probabilities and Distribution Functions in 1-D . . .6

2.2 1-D Homogeneous Markov Statistics . . . . . . . . . . 6

3.0 The Radiation/Material Temperature Equations $\ldots \ldots \ldots \ldots \ldots .8$

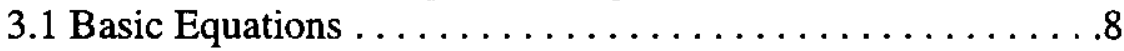

3.2 Derivation of the Restricted Average Form . . . . . . . 10

3.3 The Closure Problem .......................14

3.4 Significance of the Ensemble Average and the Variance ...15

4.0 Existing Deterministic Treatments of Stochastic Transport .......15

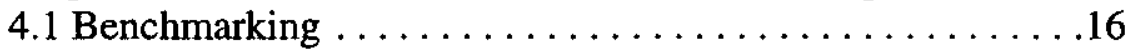

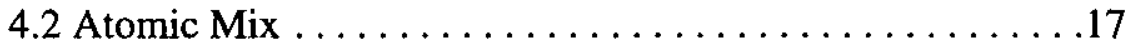

4.3 Standard Model . . . . . . . . . . . . . . . . . . . . 18

4.4 Su-Pomraning $\ldots \ldots \ldots \ldots \ldots \ldots \ldots \ldots \ldots \ldots \ldots \ldots \ldots \ldots \ldots \ldots .20$

4.5 Other Approaches $\ldots \ldots \ldots \ldots \ldots \ldots \ldots \ldots \ldots \ldots 22$

5.0 Limitations of Existing Methods $\ldots \ldots \ldots \ldots \ldots \ldots \ldots \ldots .23$

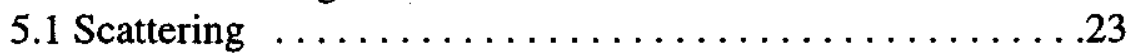

5.2 Material Temperature $\ldots \ldots \ldots \ldots \ldots \ldots \ldots \ldots .23$

5.3 General Statistics $\ldots \ldots \ldots \ldots \ldots \ldots \ldots \ldots \ldots .24$

5.4 Multi-D . . . . . . . . . . . . . . . . . . . . . 25

6.0 Summary of The Approach . . . . . . . . . . . . . . . . . 26

STOCHASTIC MODELS IN 1-D SLAB GEOMETRY ...........27

7.0 Homogeneous Markov Statistics in 1-D Slab Geometry . . . . . . .27

8.0 Formulation of the Different Methods $\ldots \ldots \ldots \ldots \ldots \ldots .28$

8.1 Benchmark - Implementation . . . . . . . . . . . . . .29

8.2 Atomic Mix - Implementation . . . . . . . . . . . . . 31

8.3 Standard Model - Implementation . . . . . . . . . . 32

8.4 Su-pomraning - Implementation . . . . . . . . . . . 33

8.4.1 Material Temperature Coupling seen as an

Effective Scatterer ...................33

8.4.2 The Su-Pomraning Closure with the

Effective Scatterer ..................35 
9.0 Numerical Results . . . . . . . . . . . . . . . . . . . . . . 37

9.1 Numerical Techniques .........................

9.2 Numerical Stability of Source Iteration $\ldots \ldots \ldots \ldots . . .39$

9.3 Results - Constant Opacities ................40

9.4 Results - Temperature Dependent Opacities . . . . . . . . .50

STOCHASTIC MODELS IN 1-D SPHERICAL GEOMETRY .......59

10.0 Markov Approximation in a 1-D Sphere $\ldots \ldots \ldots \ldots \ldots \ldots$

11.0 Formulation of the Different Methods ...............62

11.1 Benchmark - Implementation . . . . . . . . . . . .63

11.2 Atomic Mix - Implementation $\ldots \ldots \ldots \ldots \ldots \ldots .63$

11.3 Standard Model - Implementation ..............64

11.4 The Su-Pomraning Closure with the Effective

Scatterer - Implementation . . . . . . . . . . . . . . .65

12.0 Numerical Results . . . . . . . . . . . . . . . . . . . .66

12.1 Numerical Techniques $\ldots \ldots \ldots \ldots \ldots \ldots \ldots \ldots .66$

12.2 Results - Constant Opacities without Temperature

Coupling ......................... 67

12.3 Results - Constant Opacities with Temperature

Coupling ..........................

12.4 Results - Temperature Dependent opacities ..........83

CONCLUSIONS ...................................91

13.0 So What Was Learned In all This? ................91

13.1 Atomic Mix Assumptions and Spatial Connectivity ....91

13.2 Stability of Coupled Source Iteration .............92

13.3 The Role of Thermal Emission in Stochastic Transport . . .92

13.4 Correlations from Temperature Dependent Opacities ....93

13.5 Usefulness of Two-Partition Restricted Average

Operators $\ldots \ldots \ldots \ldots \ldots \ldots \ldots \ldots \ldots \ldots . . .94$

13.6 Slab Geometry Models - General Comments .........94

13.7 Spherical Geometry Models - General Comments ......95

14.0 Needed Improvements $\ldots \ldots \ldots \ldots \ldots \ldots \ldots \ldots . . . . .66$

14.1 Better Temperature Models . . . . . . . . . . . . . . . 96

14.2 Other Statistics .........................96

14.3 Other Geometries .......................97

14.4 Comparison with Experiment $\ldots \ldots \ldots \ldots \ldots \ldots .98$

15.0 Epilog $\ldots \ldots \ldots \ldots \ldots \ldots \ldots \ldots \ldots \ldots \ldots \ldots . .98$

APPENDIX A: Numerical Stability of Coupled Source Iteration ........99

16.0 Von Neumann Stability . . . . . . . . . . . . . . . . . 102

17.0 Computing the Spectral Radius $\ldots \ldots \ldots \ldots \ldots \ldots \ldots . \ldots \ldots$ 


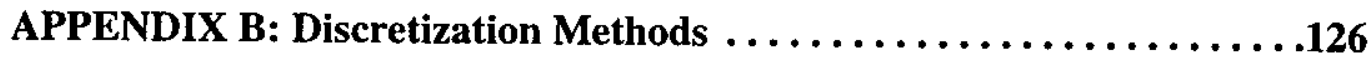

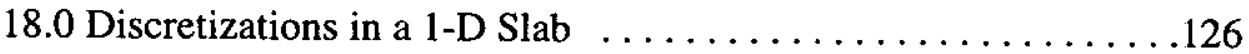

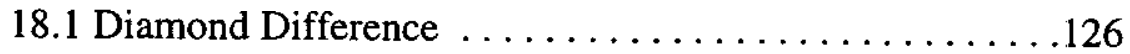

18.2 Discrete Ordinates ..................... 127

19.0 Discretizations in a 1-D Sphere $\ldots \ldots \ldots \ldots \ldots \ldots \ldots \ldots \ldots \ldots \ldots . .128$

19.1 Diamond Difference/Discrete Ordinates . . . . . . . . 128

REFERENCES $\ldots \ldots \ldots \ldots \ldots \ldots \ldots \ldots \ldots \ldots \ldots \ldots \ldots \ldots \ldots \ldots . . .131$ 



\section{INTRODUCTION AND SURVEY}

\subsection{Motivations For the Research}

It has been said that nature abhors a vacuum. It could also be said that nature loves a mess. Everywhere one looks, from the tangled tree tops to the turbulent hydrodynamics of hot mixing gasses, one is confronted with the unpredictable material distributions of the natural world. Often with these types of problems the background material is mixed in such a way that the material properties of the problem cannot be specified at any given point $\$$ except in a statistical way. And solving the differential equations of physical processes on these material-mixed domains can be anything but textbook. The mathematical nature of the problem is no longer that of simply solving a differential equation (be it difficult or easy) with specified parameters and boundary conditions. Rather, one has a differential equation with stochastic or random parameters. The solution then of our differential equation is itself a random quantity. This means that, given a correct solution to the equations of such a problem, one possesses not the exact value of a measurement but rather the expectation value or ensemble average of the measurement. This thesis is concerned with just such a problem. Specifically, this thesis is concerned with the problem of light moving through and interacting with (heating) random mixes of matter - also known as stochastic radiation transport in participating media. The specific equations of interest here are the coupled radiation transport and material temperature equations with stochastic material coefficients and sources.

In stochastic radiation transport one would like to able to answer such primary questions as, "How much light passes through the mixing region?", "How much light is 
reflected?", "How hot does it get?", and "How long does all this take?". It is these kind of predictions which connect theory to eventual experimental measurement. And it is these questions which are motivating this research from a number of different research directions. Consider below three example applications.

\subsection{Astrophysics}

One example where the transport of radiation through a random media is important is from astrophysics. Consider the following problem. A star or newly forming star is being observed from the earth, but the radiation from the star must first pass through an interstellar molecular cloud before reaching the observer, as shown in Fig. (1).

FIGUKRE 1. Án Àstrophysical Example

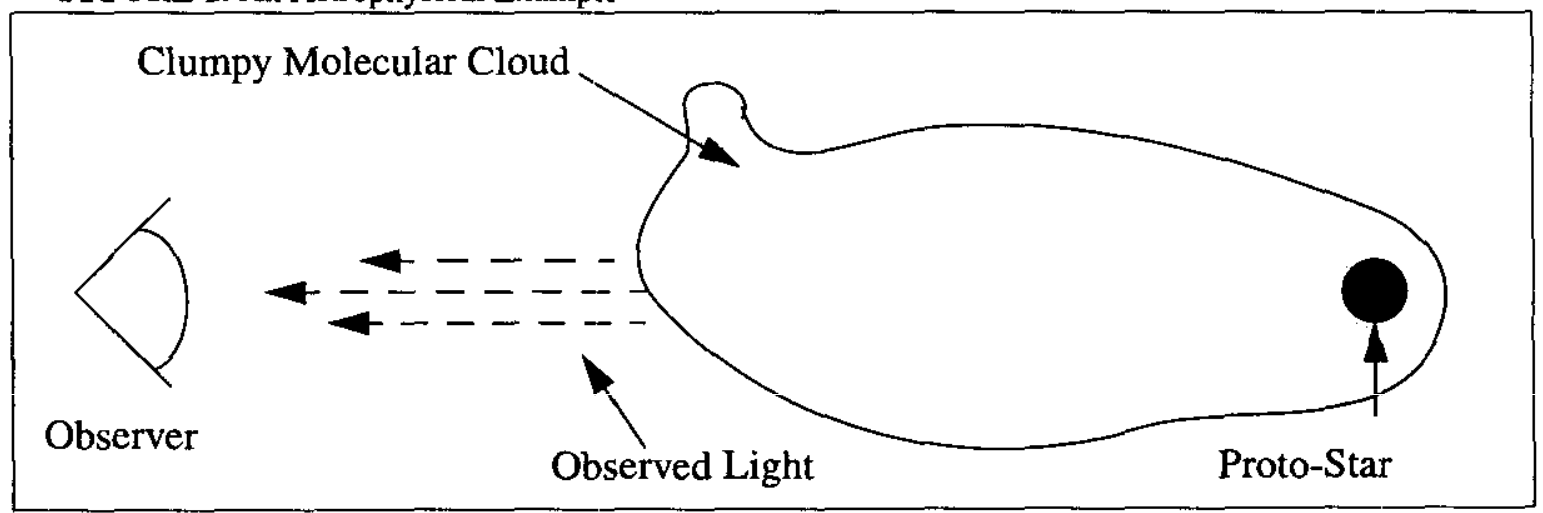

Given that the light from the star is the probe used by astronomers to gather information about processes taking place, both in the star and in the cloud itself, one must be able to understand and predict the way light moves through and interacts with the intervening interstellar cloud. But the material distributions of the interstellar clouds cannot be known in any specific detail. This prevents the precise grid resolution of any computational model of this radiation transport problem. Stated another way, any theory in astrophysics which 
uses light as a test probe depends on the ability to predict the light observed here at the earth. One then needs to be able to account for the effects of the randomly distributed material properties of the interstellar clouds through which the light must travel. For example, the random nature of the interstellar cloud structure may be important is in determining the spectral rate of photon absorption and scattering within the cloud [1]-[3].

\subsection{Atmospherics}

Another area of interest for the transport of radiation through random mixes occurs in atmospherics. Here the radiation is the energy from the sun and that energy reflected and re-radiated from the earth. The stochastic media is the earth's atmosphere.

FIGURE 2. Random Clouds in the Earth's Atmosphere

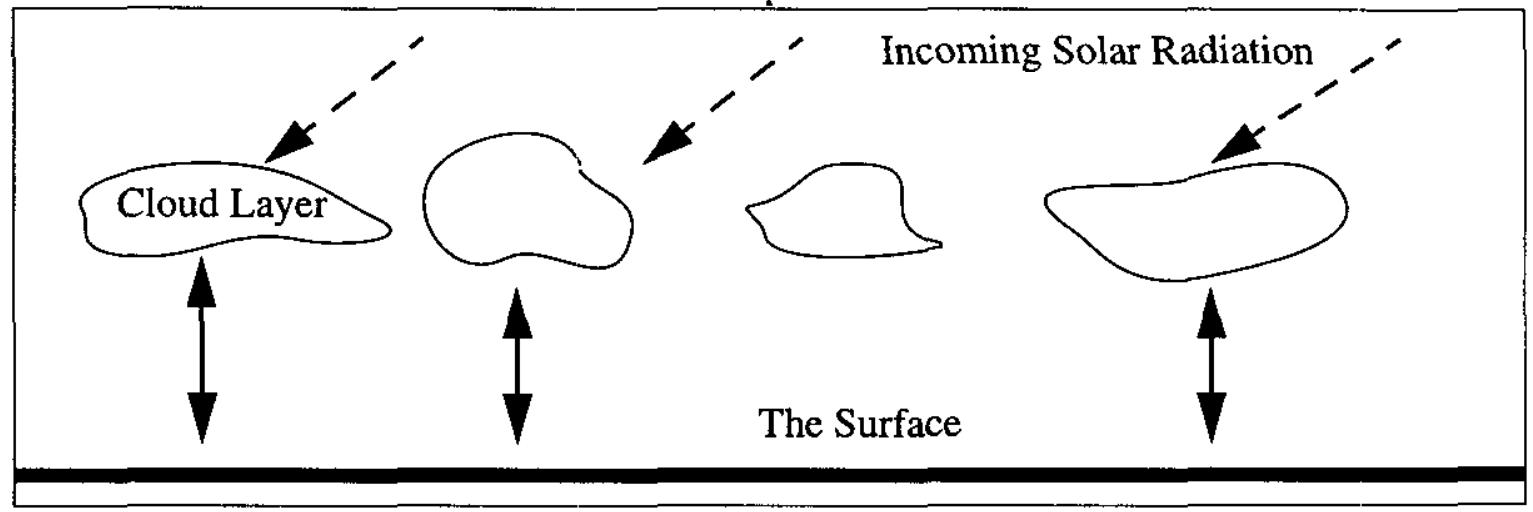

The distribution of clouds, dirt, dust, and any other suspended solids represent a stochastic material distribution. These randomly distributed materials have an effect on the way solar energy is deposited and so will effect any process related to this energy. For example climate modeling or the understanding of global warming [4],[5].

\subsection{ICF}

The field of Inertial Confinement Fusion, or ICF, is an important application for sto- 
chastic radiation transport as well. Here an intense source of X-Rays is used to implode a target. The target is typically made up of several spherical shell layers made of different materials, for example plastic and hydrogen.

FIGURE 3. Cartoon of an Imploding ICF Target

Ablator

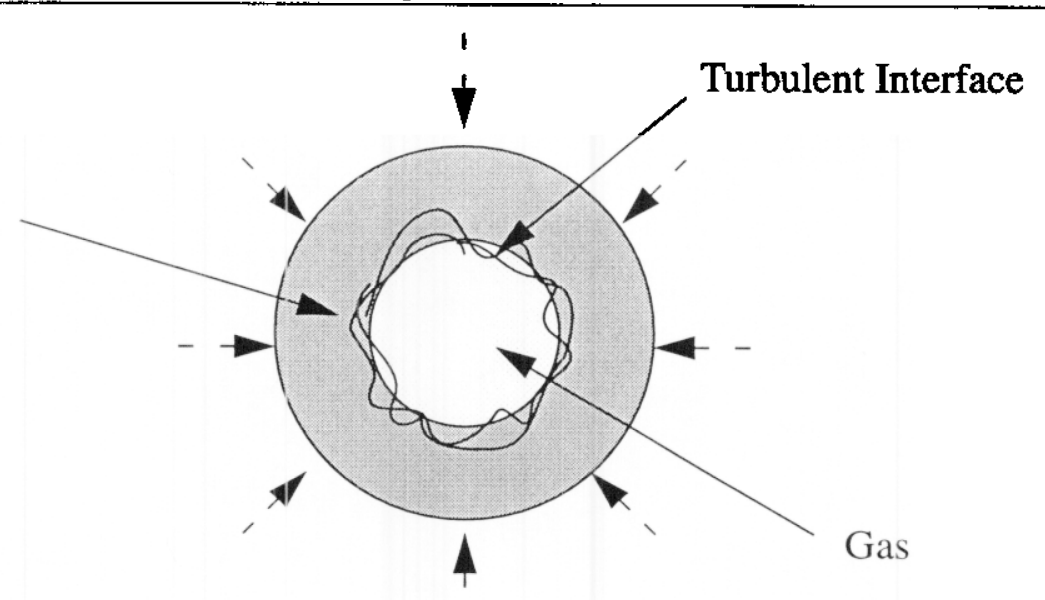

Because the different shells can have different densities, instabilities can develop at the material interfaces during implosion - specifically Raleigh-Taylor instabilities [6]-[8]. These instabilities can lead to turbulent material mixing within the target. The modeling of the radiation transport through this turbulent region is needed to accurately predict the deposition of energy and hence the hydrodynamic evolution of the system.

These are just a few of the examples where the understanding of how light transports through and interacts with a random media is important. There are many others in many different fields.

\subsection{What is a Binary Stochastic Media?}

To solve any physical problem in a random media, one must be able to quantify the ran- 
domness of the mixing. Consider a photon as it travels through a binary random media along a ray $\vec{\Omega}$, it will alternately pass through the two materials. The time it spends in each material will be dependent upon the size and shape of the chunks of the two materials, and the way in which the two materials are distributed.

\section{FIGURE 4. Idealized Random Media}

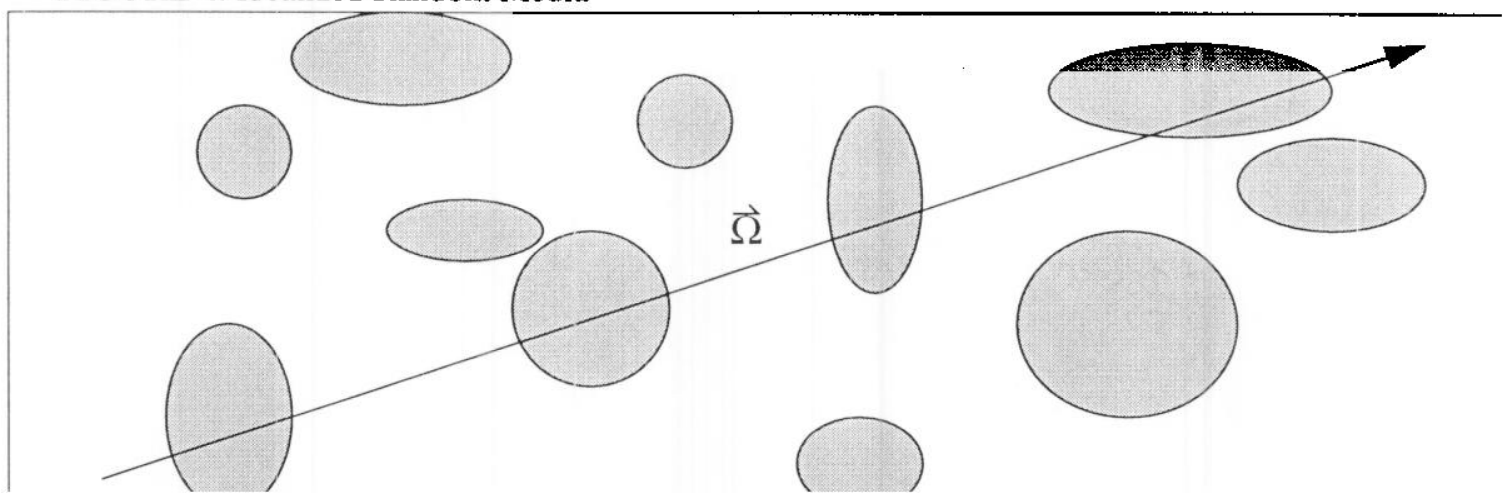

This thesis will begin with the assumption that the statistical description of the mixing is independent of position within the problem domain. This assumption gives what are known as homogeneous statistics [11]. Later, when looking at spherical geometries, nonhomogeneous statistics will be introduced. Also needed is some way to quantify the size and shape of the material chunks. The particular choice in this work is to use the probability distribution functions (herein referred to as PDF) which describe the thickness of a given material as sampled along the direction of travel $\vec{\Omega}$. These are also referred to as chunk size distribution functions. The assumption is then that sufficient information is contained within the chunk size PDF's to adequately quantify the random character of the media. Specifying a problem then is equivalent to specifying the material chunk size PDF's. 


\subsection{Absolute Probabilities and Distribution Functions in 1-D}

Consider a 1-D random media of two materials labeled material 1 and material 2. Given the PDF's for the chunk sizes of the two materials one can compute two basic properties of the media - the average size of the material chunks and the absolute probability that a randomly chosen location within the problem will contain either material 1 or material 2.

Denote the PDF for material $j$ as $f_{j}(l)$. Then the quantity $f_{j} d l$ is the probability that a randomly chosen piece of material $j$ will have a thickness between $l$ and $l+d l$ when seen along the axis of symmetry. Denote the ensemble average of a quantity with the delimiters \langle\rangle . Then the average size of a chunk of material $j$ as seen along the axis of symmetry is

$$
\left\langle l_{j}\right\rangle=\int_{0}^{\infty} l^{\prime} f_{j}\left(l^{\prime}\right) d l^{\prime} .
$$

Denote the absolute probability of observing material $j$ (for example) at a randomly chosen point anywhere within the problem as $p_{j}$. Then the $p_{j}$ are given by the formula [18]

$$
p_{j}=\frac{\left\langle l_{j}\right\rangle}{\left\langle l_{1}\right\rangle+\left\langle l_{2}\right\rangle}
$$

\subsection{1-D Homogeneous Markov Statistics}

Consider the special case of Homogeneous Markovian statistics (hereafter referred to as HM statistics) in one dimension. For Markovian statistics define the transition parameter $\Lambda_{i}$ such that the probability of transiting from material $i$ to material $j$ in a distance $d l$ 
is just $\frac{d l}{\Lambda_{i}}$ [11]. In HM statistics the transition parameter is independent of position within the problem. Then the materials have exponentially distributed chunk sizes so that the PDF's are given by

$$
f_{j}(l)=\frac{1}{\Lambda_{j}} e^{-\frac{l}{\Lambda_{j}}}
$$

Then it is easily seen that the average chunk sizes along the direction of symmetry (for example the radial direction in a 1-D sphere) are given by

$$
\left\langle l_{j}\right\rangle=\int_{0}^{\infty} \frac{l}{\Lambda_{j}} e^{-\frac{l}{\Lambda_{j}}} d l=\Lambda_{j} .
$$

Let $p_{j}$ be the absolute probabilities of a randomly chosen point being located within material $j$. Then by Eq. (2)

$$
p_{j}=\frac{\Lambda_{j}}{\Lambda_{1}+\Lambda_{2}}
$$

HM statistics have a special role in the historical development of the field of stochastic transport. For an excellent discussion of 1-D HM statistics and how it relates to stochastic transport see reference [11]. The great majority of the published work to date has been restricted to $\mathrm{HM}$ statistics and that is the case for most of the work in this thesis as well. Regardless, this thesis uses the statistical characterizations of a binary stochastic media as contained in Eqs. (3), (4) and (5). The main reason for this is it enables more easily formal extension of existing work. 


\subsection{The Radiation/Material Temperature Equations}

Consider now the radiation transport and material temperature equations and some of the existing methods for solving these equations in a stochastic media.

\subsection{Basic Equations}

Restrict the problem to that of grey radiation transport. This means that there will not be coupling between radiation of different wavelengths and the flux of radiation is described by a single equation for the energetic average or grey equivalent. The main reason for doing this here is to simplify the writing of computer codes to solve the equations. Ultimately one would like to solve the problem in complete generality but that is beyond the scope of this thesis. This is but the first step towards the inclusion of the new physics of material temperature coupling to the deterministic radiation transport problem. The two basic equations which describe grey radiation transport coupled to matter temperature are

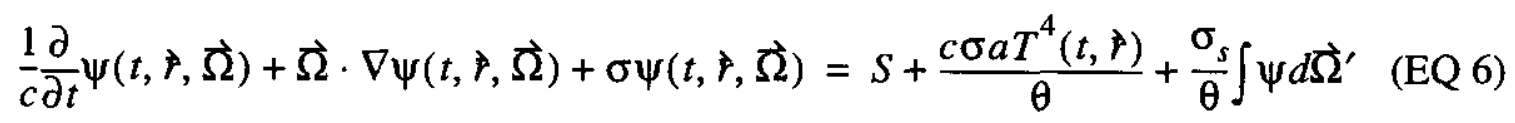

and

$$
\rho C_{\nu} \frac{\partial}{\partial t} T(t, \grave{l})=-c \sigma a T^{4}(t, \grave{\imath})+\left(\sigma-\sigma_{s}\right) \int \psi d \vec{\Omega},
$$

where $\psi(t, \grave{r}, \grave{\Omega})$ is the radiation specific intensity and $T$ is the material temperature. The quantities $t, \vec{r}$, and $\vec{\Omega}$ are the temporal, spatial and angular coordinates, $S$ is a general radiation source, $c$ is the speed of light, $a$ the radiation constant, $\rho$ the mass density, and 
$C_{v}$ the heat capacity. The integrals are taken over all directions. The $\theta$ is a dimensionality constant given by $\theta=\int d \overrightarrow{\Omega^{\prime}}$.

The total and scattering coefficients are given by $\sigma$ and $\sigma_{s}$. Because the background media is randomly mixed, the coefficients $\sigma(t, \grave{l})$ and $\sigma_{s}(t, \grave{l})$ are stochastic quantities. Then radiation specific intensity $\psi$ and the material temperature $T$ are themselves continuous stochastic quantities in that they can take on a continuous range of possible values depending on the particular realization of the mixing statistics. One then is interested in obtaining as a solution the ensemble average of $\psi$ and the ensemble average of $T$ which is denoted as $\langle\psi\rangle$ and $\langle T\rangle$ respectively.

Notice that the equations have fourth order terms in the matter temperature $T$. To further simplify the problem a bit, assume a particular analytic form for the heat capacity $C_{v}$. Assume

$$
C_{v}=\frac{4 a}{\rho} T^{3}
$$

This form for the heat capacity is commonly used to simplify problems involving radiation coupled to material temperature. It has the nice property that it makes the material temperature equation linear. Define the quantity

$$
\phi(t, z)=a T^{4}
$$

which is the material temperature energy density. Then Eqs. (6) and (7) become 


$$
\frac{1}{c} \frac{\partial}{\partial t} \psi(t, \vec{r}, \vec{\Omega})+\vec{\Omega} \cdot \nabla \psi(t, \vec{r}, \vec{\Omega})+\sigma \psi(t, \vec{\jmath}, \vec{\Omega})=S+\frac{c \sigma \phi(t, \vec{P})}{\theta}+\frac{\sigma_{s}}{\theta} \int \psi d \vec{\Omega}^{\prime}
$$

and

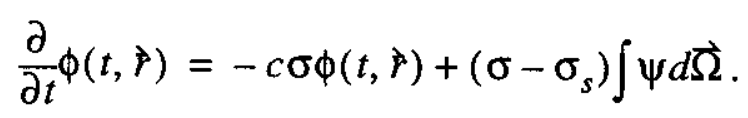

Equations (10) and (11) are now linear in the two quantities $\psi$ and $\phi$. The task at hand is to solve these two coupled stochastic and time evolving differential equations for $\langle\psi\rangle$ and $\langle T\rangle$.

\subsection{Derivation of the Restricted Average Form}

Consider what happens if one takes the ensemble average of Eqs. (10) and (11). This gives

$$
\frac{1}{c} \frac{\partial}{\partial t}\langle\psi\rangle+\vec{\Omega} \cdot \nabla\langle\psi\rangle+\langle\sigma \psi\rangle=S+\frac{c\langle\sigma \phi\rangle}{\theta}+\frac{1}{\theta} \int\left\langle\sigma_{s} \psi\right\rangle d \vec{\Omega}^{\prime}
$$

and

$$
\frac{\partial}{\partial t}\langle\phi\rangle=-c\langle\sigma \phi\rangle+\int\left\langle\left(\sigma-\sigma_{s}\right) \psi\right\rangle d \vec{\Omega}
$$

These equations are not of a simple form. The terms which are averages of products (such as $\langle\sigma \phi\rangle$ for example) change the form of the equations to that of non-linear ones.

A similar but different averaging approach to the solution of Eqs. (10) and (11) is to introduce a special operator, the restricted ensemble averaging operator. This operator computes the ensemble averaging of a quantity over a restricted subset of the total possible 
realizations of the statistics, instead of all realizations. The advantage is that it retains more linearity (so to speak) for the differential equations. Consider a random spatial distribution of two materials. Then at any point within the spacial domain of the problem there will either exist material 1 or material 2 with probability $p_{1}$ or $p_{2}$ respectively given by Eq. (5). Define now a the function $\chi_{i}$ such that $\chi_{i}(\vec{r})=1$ if material $i$ is located at $\vec{r}$, else $\chi_{i}(r)=0$. Then the ensemble average of $\chi_{i}$ is just the probability of being in material $i$ at a random location. In other words

$$
\left\langle\chi_{i}\right\rangle=p_{i}
$$

And for any random quantity $Q(r)$ which depends on these same binary statistics one has that

$$
\left\langle\chi_{i} Q\right\rangle=p_{i} Q_{i}
$$

and

$$
\langle Q\rangle=p_{1} Q_{1}+p_{2} Q_{2}
$$

The quantity $Q_{i}(\vec{r})$ is then the average value of $Q$ over the subset of possible realizations which have material $i$ located at $\vec{r}$, i.e. the restricted ensemble average of $Q$. Then the multiplication of a quantity by $\chi_{i}$ and subsequent averaging over all possible realizations acts as a restricted ensemble average operator [14],[15]. The average of the opacities becomes 


$$
\langle\sigma\rangle=p_{1} \sigma_{1}+p_{2} \sigma_{2}
$$

and

$$
\left\langle\sigma_{s}\right\rangle=p_{1} \sigma_{s 1}+p_{2} \sigma_{s 2},
$$

where $\sigma_{i}$ and $\sigma_{s i}$ are the total and scattering coefficients for material $i$. One can now apply the restricted ensemble average operator to Eq. (10). Assuming that the opacities are constant one gets

$$
\frac{1}{c} \frac{\partial}{\partial t}\left(p_{i} \psi_{i}\right)+\vec{\Omega} \cdot\left\langle\chi_{i} \nabla \psi\right\rangle+\sigma_{i}\left(p_{i} \psi_{i}\right)=p_{i} S_{i}+\frac{c \sigma_{i} p_{i} \phi_{i}}{\theta}+\frac{\sigma_{s}}{\theta} \int\left(p_{i} \psi_{i}\right) d \vec{\Omega}^{\prime},
$$

with $i=1,2$. The restricted ensemble averaging commutes with all the operators in Eq. (10) except for the gradient operator [15]. Integrate the gradient term by parts so that

$$
\left\langle\chi_{i} \nabla \psi\right\rangle=\left\langle\nabla\left(\chi_{i} \psi\right)-\psi \nabla \chi_{i}\right\rangle=\nabla\left(p_{i} \psi_{i}\right)-\left\langle\psi \nabla \chi_{i}\right\rangle
$$

Using Eq. (20) in Eq. (10) gives a transport-like equation for each material 1 and 2.

$$
\frac{1}{c} \frac{\partial}{\partial t}\left(p_{i} \psi_{i}\right)+\vec{\Omega} \cdot\left(p_{i} \psi_{i}\right)+\sigma_{i}\left(p_{i} \psi_{i}\right)=p_{i} S_{i}+\frac{c \sigma_{i} p_{i} \phi_{i}}{4 \pi}+\frac{\sigma_{s}}{4 \pi} \int\left(p_{i} \psi_{i}\right) d \vec{\Omega}^{\prime}+\left\langle\psi \nabla \chi_{i}\right\rangle
$$

Restricted ensemble averaging of the temperature Eq. (11) gives

$$
\frac{\partial}{\partial t}\left(p_{i} \phi_{i}\right)=-c \sigma_{i}\left(p_{i} \phi_{i}\right)+\left(\sigma_{i}-\sigma_{s i}\right) \int\left(p_{i} \psi_{i}\right) d \vec{\Omega}
$$

Now Eqs. (21) and (22) are a set of four equations whose solutions combine to give the solution to the coupled radiation material temperature problem when the opacities are constant. The ensemble average solutions explicitly in terms of the solutions to Eqs. (21) and 
(22) are

$$
\langle\psi\rangle=p_{1} \psi_{1}+p_{2} \psi_{2}
$$

and

$$
\langle\phi\rangle=p_{1} \phi_{1}+p_{2} \phi_{2}
$$

But what about that new term which has appeared on the right hand side of the radiation transport equation? Refer to this new term, for reasons which will become clear, as the statistical coupling term. This method has retained the linearity of all of the terms in the equations, with the possible exception of the new coupling term, whatever it may be. This process of applying the restricted ensemble average operator has served to capture all of the statistical nature of the equations and move it all into the coupling term, leaving the rest of the equations linear. The trick of course is calculating what that $\left\langle\Psi \nabla \chi_{i}\right\rangle$ coupling term is.

Notice, Eqs. (21) and (22) have a nice form because the material coefficients $\sigma_{i}$ and $\sigma_{s i}$ were specified as constant for each material. Should they be dependent upon position or time (as they would be in real temperature dependent material), then Eqs. (21) and (22) become approximations. This is because the procedure for getting Eqs. (21) and (22) depends on the fact that all of the possible statistical realizations can be split into two subsets within which the material properties are constant. It is precisely because the set of possible realizations can be partitioned into these two convenient subsets that the ensemble averages of Eqs. (10) and (11) can each be split into two sets of coupled transport-temperature equations - one for the average over each of the two subsets. Should the material 
properties be continuously variable, the procedure leading to Eqs. (21) and (22) would yield not four equations (coupled temperature and transport equations for each of the two materials), but an infinite number (coupled temperature and transport equations for every possible value that the opacities can attain). But for constant material properties Eqs. (21) and (22) apply.

The main advantage to this approach is that the numerical inversion of the left hand side of Eq. (21) is now amenable to the same techniques used for the solution of ordinary deterministic transport equations. One does not need to develop new numerical solution techniques to solve these new equations. This is an important theme central to the methods used in this thesis.

\subsection{The Closure Problem}

Determination of the $\left\langle\Psi \nabla \chi_{i}\right\rangle$ coupling term, whether in exact or approximate form, is

referred to as the closure problem. The function $\chi_{i}(\not)$ is a step function in space and has a zero gradient everywhere except at the interface between two materials. The $\left\langle\Psi \nabla \chi_{i}\right\rangle$ term is then the average of the specific intensity over all realizations where an interface between material 1 and material 2 lies at position $\gg$. It is not clear yet exactly how one computes this in a general geometry or with a general set of statistics. However, this value has been computed exactly for the special case of Markov statistics and in a slab or rod geometry [11]. It is this success which encourages further investigations into the use of the restricted ensemble averaging operator technique. This is further elaborated in Section 4.3. 


\subsection{Significance of the Ensemble Average and the Variance}

Consider for a moment what the ensemble average of any statistical quantity represents in the context of a real experiment, say the force exerted by raindrops on a plate placed out in the rain. The net force exerted by the raindrops at any given instant in time will be a statistically random quantity. The average of this force over time will be the ensemble average. But how well does this average represent the force as measured at any given time? In other words, how well does the ensemble average of a quantity represent the quantity as measured for any individual statistical realization? The answer is addressed by a quantity called the variance. The variance is a measure of how much the measurement from any individual statistical realization can be expected to vary from the average. In other words, how good is the average at representing a typical measurement? The root mean square variance is computed as

$$
\operatorname{var}(A)=\sqrt{\left\langle A^{2}\right\rangle-\langle A\rangle^{2}},
$$

where the quantity $A$ here is just some statistical quantity. So for a statistical problem with a large variance, the ensemble average will be a poor representation of an individual statistical measurement. This is why when computing stochastic solutions, it is important to get a handle on what the actual variance is, if possible. It tells the experimenter if the expectation value is in fact what he or she should expect to measure.

\subsection{Existing Deterministic Treatments of Stochastic Transport}

In this section is discussed several of the methods which have been developed in the lit- 
erature to address the problem of stochastic transport without material temperature. The extension of these methods to include the coupling to material temperature in a stochastic setting (and subsequent testing of the methods) is the core work of this thesis.

\subsection{Benchmarking}

Let us assume for the moment that one has a model for the solution to Eqs. (21) and (22). This is equivalent to saying one has an explicit form for the $\chi_{i}(\grave{)})$ term. How would one test the model? How would one know the model to be correct, or not? There is a brute force technique for the solution of Eqs. (10) and (11) in a stochastic media. It is a Monte Carlo type of method in that it uses the statistical description of the problem to generate many different sample realizations of the material distributions. It is not strict Monte Carlo though in that it does not generate individual flight histories of individual photons. Rather it computes the deterministic solution to Eqs. (10) and (11) over many different realizations and then averages all of the so computed deterministic solutions. It generates ensemble averages in the true sense of the word. This is referred to as the Benchmarking Process [14]. The ensemble average solutions for $\langle\psi\rangle$ and $\langle T\rangle$ computed in this manner become exact in the limit of sampling an infinite number of the statistical realizations. This obviously is not a reasonable thing to do very often as it involves computing the solution to a coupled set of differential equations an infinite (or very large) number of times. Doing it once is expensive enough! This makes the benchmarking process prohibitively expensive for most uses. However, given the accuracy of the method, it is very useful for verifying other models - which hopefully will be much faster and less expensive, if less accurate. 


\subsection{Atomic Mix}

There is a popular method commonly referred to as the atomic mix model. This method has the advantage of being simple to formulate, easy to implement, and it's computational expense is just that of a single standard deterministic solution of Eqs. (10) and (11). Consider the ensemble averages of the product terms in Eqs. (12) and (13). Do a decomposition of the random quantities into a mean and an oscillating part so that

$$
\begin{aligned}
& \psi=\langle\psi\rangle+\tilde{\psi} . \\
& \varphi=\langle\varphi\rangle+\tilde{\varphi}
\end{aligned}
$$

where the oscillating parts $\tilde{\psi}$ and $\tilde{\varphi}$ have zero mean. Do likewise for $\sigma$ and $\sigma_{s}$. Then (for example)

$$
\langle\sigma \psi\rangle=\langle\sigma\rangle\langle\psi\rangle+\langle\tilde{\sigma} \tilde{\psi}\rangle
$$

and

$$
\langle\sigma \varphi\rangle=\langle\sigma\rangle\langle\varphi\rangle+\langle\tilde{\sigma} \tilde{\varphi}\rangle
$$

The atomic mix assumption then is that the average of the cross correlation terms like $\langle\tilde{\sigma} \tilde{\psi}\rangle$ are all zero. This assumption yields

$$
\langle\sigma \psi\rangle=\langle\sigma\rangle\langle\psi\rangle
$$

and

$$
\left\langle\sigma_{s} \psi\right\rangle=\left\langle\sigma_{s}\right\rangle\langle\psi\rangle
$$


with similar equations for averages of products with $\varphi$. Then Eqs. (12) and (13) reduce to

$$
\frac{1}{c} \frac{\partial}{\partial t}\langle\psi\rangle+\vec{\Omega} \cdot \nabla\langle\psi\rangle+\langle\sigma\rangle\langle\psi\rangle=\langle S\rangle+\frac{c\langle\sigma\rangle\langle\phi\rangle}{\theta}+\frac{\left\langle\sigma_{s}\right\rangle}{\theta} \int\langle\psi\rangle d \vec{\Omega}^{\prime},
$$

and

$$
\frac{\partial}{\partial t}\langle\phi\rangle=-c\langle\sigma\rangle\langle\phi\rangle+\left\langle\sigma-\sigma_{s}\right\rangle \int\langle\psi\rangle d \vec{\Omega}
$$

Physically this is equivalent to making the assumption that within a small volume $d V$ located anywhere in the problem, the material is well represented by the average of the two materials locally, and that the radiation specific intensity and material temperature are completely independent of any structure or statistical correlation between the two materials. Eqs. (32) and (33) are now ordinary linear equations for $\langle\psi\rangle$ and $\langle T\rangle$ which can be solved using the usual methods for solution of Eqs. (10) and (11). These are nice properties and it is for this reason that this technique is widely used. However these are rather crude approximations in many cases. As will be shown later in the numerical simulations, for certain cases the atomic mix approximation has quite a large error when compared to the benchmark solutions.

\subsection{Standard Model}

This method was originally developed to solve the steady state stochastic transport equation, i.e. Eq. (10) for steady state and no coupling to material temperature. It does not in it's original form include any treatment of material temperature. This model was inde- 
pendently developed by several authors [10]-[13] but is commonly referred to as the Levermore-Pomraning model. This work refers to this model as the Standard Model. It gives the ensemble average solution for the equation

$$
\vec{\Omega} \cdot \nabla \psi(t, \vec{\digamma}, \vec{\Omega})+\sigma \psi(t, \vec{\digamma}, \vec{\Omega})=S+\frac{\sigma_{s}}{\theta} \int \psi d \vec{\Omega}^{\prime}
$$

as

$$
\langle\psi\rangle=p_{1} \Psi_{1}+p_{2} \psi_{2}
$$

where the $\Psi_{i}$ are found via the solution of the two coupled equations

$$
\vec{\Omega} \cdot \nabla\left(p_{i} \psi_{i}\right)+\sigma\left(p_{i} \psi_{i}\right)=p_{i} S_{i}+\frac{\sigma_{s}}{\theta} \int\left(p_{i} \psi_{i}\right) d \vec{\Omega}^{\prime}+\frac{p_{j} \psi_{j}}{\Lambda_{j}(\vec{\Omega})}-\frac{p_{i} \psi_{i}}{\Lambda_{i}(\vec{\Omega})}
$$

where $i, j=1,2$ and $i \neq j$. If the problem is further restricted to a 1-D purely absorbing media with a HM statistical description, then this model becomes an exact solution [11],[15]. This model can be thought of as a special case of Eq. (21) with a particular choice of closure relations for the statistical coupling terms. In other words the standard model Eqs. (34) through (36) has the closure relation

$$
\left\langle\psi \nabla \chi_{i}\right\rangle=\frac{p_{j} \Psi_{j}}{\Lambda_{j}(\bar{\Omega})}-\frac{p_{i} \Psi_{i}}{\Lambda_{i}(\vec{\Omega})}
$$

This model has been shown to be quite robust and fairly accurate [14]. However, with the inclusion of scattering, this is no longer an exact model. One would like to be able to include the statistical effects of scattering for solving a larger class of problems. One way 
to address this issue is discussed next.

\subsection{Su-Pomraning}

In an attempt to build on the success of the Standard Model for the obtaining the stochastic solution of Eq. (34), Su and Pomraning have proposed two alternatives to the closure relation Eq. (37) [16]. These new closures are aimed at improving the performance of the Standard Model in cases when scattering is present. Both of the new closures relations are of the general form

$$
\left\langle\psi \nabla \chi_{i}\right\rangle=\kappa\left[\frac{\left(p_{j} \psi_{j}\right)}{\Lambda_{j}(\vec{\Omega})}-\frac{\left(p_{i} \psi_{i}\right)}{\Lambda_{i}(\vec{\Omega})}\right]+\frac{\eta}{\theta}\left[\frac{\int\left(p_{i} \psi_{i}\right) d \vec{\Omega}^{\prime}}{\Lambda_{i}(\vec{\Omega})}-\frac{\int\left(p_{j} \psi_{j}\right) d \vec{\Omega}^{\prime}}{\Lambda_{j}(\vec{\Omega})}\right]
$$

Physically one can think of these coupling terms as a kind of stochastic source/sink of particles. They represent the gain and loss of photons as they stream across the randomly distributed material interface boundaries (linear terms) and get randomly redistributed in angle via the scattering-like process (integral terms).

So the solution is achieved by solving the two coupled equations

$$
\vec{\Omega} \cdot \nabla\left(p_{i} \psi_{i}\right)+\sigma\left(p_{i} \psi_{i}\right)=p_{i} S_{i}+\frac{\sigma_{s}}{\theta} \int\left(p_{i} \psi_{i}\right) d \vec{\Omega}^{\prime}+\left\langle\psi \nabla \chi_{i}\right\rangle
$$

where $i, j=1,2$ and $i \neq j$ with the closure Eq. (38) and

$$
\langle\psi\rangle=p_{1} \psi_{1}+p_{2} \psi_{2}
$$

The new parameters $\eta$ and $\kappa$ are positive weighting constants which are somehow meant 
to take into account the effects that scattering has on the evolution of the statistical solution. Notice that if $\eta=0$ and $\kappa=1$ this closure recovers the Standard Model. These two new closures each specify different values for the two parameters $\eta$ and $\kappa$. The first of these closures is given by

$$
\eta=\frac{\sqrt{\left\langle\sigma_{a}\right\rangle\langle\sigma\rangle}\left[\left\langle\sigma_{a}\right\rangle\left(\sigma_{1}-\sigma_{2}\right)^{2}+\langle\sigma\rangle\left(\sigma_{a 1}-\sigma_{a 2}\right)^{2}\right]}{\left[\left\langle\sigma_{a}\right\rangle\left(\sigma_{1}-\sigma_{2}\right)\right]^{2}+\left[\langle\sigma\rangle\left(\sigma_{a 1}-\sigma_{a 2}\right)\right]^{2}}
$$

and

$$
\kappa=0 .
$$

Here $\sigma_{a i}$ is the absorption opacity for material $i$ and is given by

$$
\sigma_{a i}=\sigma_{i}-\sigma_{s i}
$$

And $\left\langle\sigma_{a}\right\rangle$ is the ensemble average of the absorption opacity given by

$$
\left\langle\sigma_{a}\right\rangle=p_{1} \sigma_{a 1}+p_{2} \sigma_{a 2}
$$

The second of these "scattering accountable" closures proposed by Su and Pomraning is given by

$$
\eta=\frac{1}{\sqrt{1-\frac{\left\langle\sigma_{s}\right\rangle}{\langle\sigma\rangle}}}
$$

and 


$$
\kappa=\frac{\frac{\left\langle\sigma_{s}\right\rangle}{\langle\sigma\rangle}}{\sqrt{1-\frac{\left\langle\sigma_{s}\right\rangle}{\langle\sigma\rangle}}}
$$

Notice that for both of these new closures, when scattering is zero, they both recover the standard model, i.e $\eta=1$ and $\kappa=0$. The closure given by Eqs. (38) through (42) and the closure in Eqs. (38), (41) and (42) are both derived by the same method which is outlined here. First assume the form in Eqs. (38) and (39). Then assume that the correlation length $\lambda_{c}$, defined as

$$
\frac{1}{\Lambda_{c}}=\frac{1}{\Lambda_{1}}+\frac{1}{\Lambda_{2}}
$$

is small. Next assume that the fluctuations of the radiation specific intensity are small. In other words

$$
\psi=\langle\psi\rangle+\tilde{\psi} \varepsilon
$$

where $\varepsilon$ is a smallness parameter. Then, equate the limiting (first order) cases of $\Lambda_{c}$ « 1 in Eqs. (38) and (39) with $\varepsilon$ « 1 in a direct averaging of Eq. (34). Assuming $\kappa=0$ gives Eq. (41). The Eqs. (45) and (46) are obtained by assuming $\sigma_{1}=\sigma_{2}$ and $\sigma_{s 1}=\sigma_{s 2}$ (clearly an undesirable assumption). For a detailed derivation please refer to reference [16]. This model also did not have any treatment of material temperature.

\subsection{Other Approaches}

The methods listed above were chosen for study in this work because they all lend 
themselves to numerical solution by way of common deterministic transport solution techniques. That is why Monte Carlo approaches were not considered here, although others have used Monte Carlo methods for stochastic transport problems [1],[19],[21]. However, aside from the deterministic methods listed above, there are several other ways to approach the problem of deterministic stochastic transport. For example the Method of Smoothing [9] and the Liouville Master Equation [12]. For a good discussion of these and other alternative deterministic formulations see reference [11].

\subsection{Limitations of Existing Methods}

All of the above listed methods have their limitations.

\subsection{Scattering}

None of the above methods except for the benchmark process can exactly treat the effects of scattering on stochastic transport. Without scattering, the average of a quantity like the radiation specific intensity only depends upon the possible states of the system back along that particular ray $\bar{\Omega}$ in space. Introduce scattering however and the same average is now coupled to the past flight history states over all possible directions. This greatly complicates the problem and the evaluation of averages. For a general discussion of the scattering problem see reference [11].

\subsection{Material Temperature}

None of the above methods can account for the inclusion and time evolution of a material temperature coupled to the radiation. The coupling of radiation intensity to material 
temperature makes the problem harder for several reasons. Reason number one is the redistribution in angle of photons due to thermal re-emission. This correlation through all angles creates coupling to the past flight history states over all possible directions, just as in the case with scattering.

With the inclusion of material temperature there are two continuous stochastic quantities to be found instead of one. This presents the daunting task of simultaneously solving two coupled stochastic and non-linear partial differential equations. Deutsch and Vanderhaegen [6] have considered the case for 1-D radiation transport with the two materials at two different but fixed temperatures and fixed opacities, i.e. as binary stochastic quantities. This is equivalent to having binary stochastic radiation sources of the form $\sigma B$ where there are non-zero statistical correlations between the total opacity $\sigma$ and $B$, with $B$ some function of the material temperature. But this work is concerned with the more general case of a time varying material temperature.

And lastly if the material properties are allowed to change with temperature, the opacities are now continuous rather than binary stochastic quantities. This introduces strong new correlations which need to be accounted for in the calculation of averages.

Much of the effort in this work is dedicated to exploring how to include material temperature with stochastic transport methods and what new problems it introduces.

\subsection{General Statistics}

All the above treatments make very strong assumptions about the statistical nature of the material mixing. One would of course like to be able to have that statistical description be specified by the problem at hand, and not be required to impose the assumption of a 
certain statistical description on the problem. For example, it has been suggested that turbulence in fluids is described by Levy power law statistics rather than Markov statistics [21]. A technique based on the ideas of Renewal Theory has been proposed [11] for particle transport through a random media with general statistical description. Unfortunately this formalism does not easily lend itself to solution via standard transport solution techniques.

\subsection{Multi-D}

The problem of formulating and testing a stochastic transport model in arbitrary dimensions or geometries is closely related to the problem of general statistics. Assume for the moment that one has a general model in some geometry. To test it you need to be able to generate benchmarks against which to compare the model (odds are no analytic solution will exist). This means that one must first be able to (somehow) generate a computational mesh which represents a single statistical realization of the mixing statistics. While it is not known how to do this in two and three dimensions, approximate methods have been suggested [1]. However it is clear how to generate rod and slab geometry mixes [14],[20]. It may also be possible to generate two and three dimensional Markovian mixes [23]. But for non-Markovian statistics in a general geometry this remains unsolved. So there is the

problem of verifying the accuracy of a model by not being able to compute a benchmark. However, one could generate some set of benchmark meshes and then do ray tracing on these to determine their statistical nature. But then one needs to be able to incorporate into the model whatever statistics arise from this procedure and we are back to the general statistics problem. As a first step toward exploring new possibilities, the 1-D spherical geom- 
etry is considered in Section 10.0 .

\subsection{Summary of The Approach}

This thesis tries to solve the radiation transport equation coupled to the material temperature equation in a stochastic media. The benchmark process described in Sec. (4.1) is used to generate the "true" answers. The atomic mix method Sec. (4.2), is used to explore how well this common method works in this context. Then, beginning with the Standard Model Sec. (4.3), a new method is proposed which generalizes the Standard Model to include coupling to material temperature. Then is proposed a second new method which is a generalization of the Su-Pomraning model, Sec. (4.4), again to include the coupling to material temperature. The performance of the four methods is explored over a range of parameter spaces and for two different geometries, 1-D slab geometry and 1-D spherical geometry.

Also, the Standard Model has never before been used in a 1-D sphere. So this was investigated with and without the inclusion of the material temperature coupling.

The implementation and generalization of these methods to the 1-D slab geometry if presented in Sec. (8.0). The implementation and generalization of these methods to the 1D spherical geometry is presented in Sec. (11.0). 


\section{STOCHASTIC MODELS IN 1-D SLAB GEOMETRY}

\subsection{Homogeneous Markov Statistics in 1-D Slab Geometry}

In this chapter is proposed some new methods for modeling the stochastic solution to Eqs. (10) and (11) in a 1-D slab geometry. Also presented are the formulations for some standard methods for which 'numerical solutions are computed for comparison. The formulations of these different models are presented in Sec. (8.0) and the numerical solution results in Sec. (9.0). In this chapter the problem is restricted to a purely absorbing media in a 1-D slab geometry. In this geometry the media is composed of alternating slabs of the two materials, as shown in Fig. (5).

FIGURE 5. Typical 1-D Slab Geometry Media

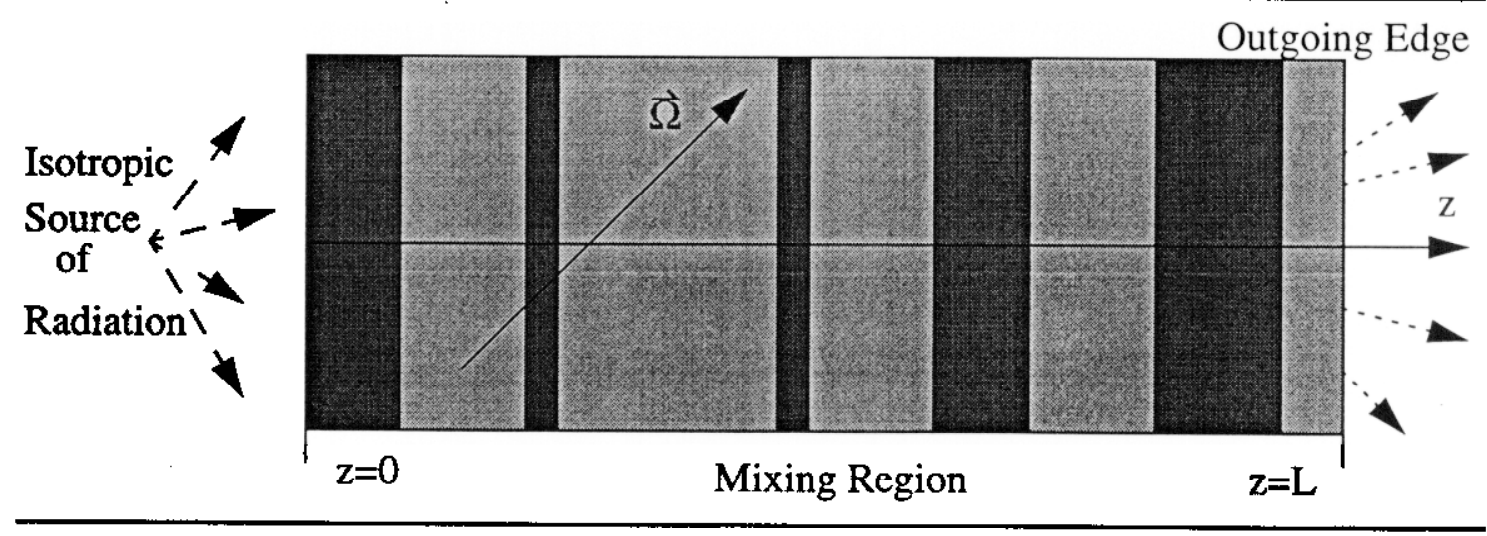

The problem is assumed to be described by HM statistics so that the PDF for the chord lengths of material $i$ (along the $\mathrm{z}$ direction) are given by

$$
f_{i}(z)=\frac{1}{\lambda_{i}} e^{-\frac{z}{\lambda_{i}},}
$$


where $\lambda_{i}$ is then the average chord length of material $i$ along the $z$ direction. Let $\mu$ be the cosine of the angle between the ray $\vec{\Omega}$ and the $z$ direction. Then the average size of a chunk of material $i$ as seen along the ray $\vec{\Omega}$ is given buy

$$
\Lambda_{i}(\vec{\Omega})=\frac{\lambda_{i}}{|\mu|}
$$

The probability of finding material $i$ at any given point is then given by

$$
p_{i}=\frac{\lambda_{i}}{\left(\lambda_{1}+\lambda_{2}\right)}
$$

\subsection{Formulation of the Different Methods}

One wishes to obtain the stochastic solution, i.e the ensemble averages of the radiation specific intensity $\langle\psi\rangle$ and the material temperature energy density $\langle\varphi\rangle$, in the 1-D HM media shown in Fig. (5) and described by Eqs. (49) and (51). In a purely absorbing, source-free, 1-D slab geometry Eqs. (10) and (11) become

$$
\frac{1}{c} \frac{\partial}{\partial t} \psi(t, z, \mu)+\mu \frac{\partial}{\partial z} \psi(t, z, \mu)+\sigma_{a} \Psi(t, z, \mu)=\frac{c \sigma_{a}}{2} \phi(t, z)
$$

and

$$
\frac{\partial}{\partial t} \phi(t, z)=-c \sigma_{a} \phi(t, z)+\sigma_{a} \int_{-1}^{1} \psi(t, z, \mu) d \mu
$$

where $\sigma_{a}$ is the absorption opacity. Because the materials are purely absorbing, $\sigma=\sigma_{a}$ 
and $\sigma_{s}=0$ (this no-scattering assumption is appropriate for X-ray transport in matter, for example). These simplifying assumptions were made to make the problem more tractable while still retaining some of the new difficulties associated with the addition of material temperature coupling. The assumptions resulting in Eqs. (52) and (53) are exactly those assumptions under which the Standard Model as presented in Sec. (4.3) is exact when material temperature coupling is absent. In other words the goal here is to see what new issues arise with the addition of new physics without making the problem overly complicated.

\subsection{Benchmark - Implementation}

As described in Sec. (4.1), the benchmarking process is not so much a model as a procedure for the numerical evaluation of the true ensemble solution to the stochastic problem. It is not new and it requires no reformulation, needing only the numerical techniques for the deterministic solution of Eqs. (52) and (53). It is included here because, though expensive, it provides accurate ensemble average solutions to the problem. The procedure is simple. One uses the PDF's given by Eq. (49) to populate a particular realization of the statistics. One then determines the solution to Eqs. (52) and (53) on that particular realization. The problem is repopulated with a new material realization and the equations solved again. This process is repeated many times and the resulting many solutions are averaged. This gives an ensemble average in the truest sense of the word.

But how does one generate a particular realization of the statistics? Let $P_{i}\left(x_{i} \leq x\right)$ be the probability that a randomly chosen piece of material $i$ has a chord length less than or equal to $x$ as seen along the $z$ axis. Then 


$$
P_{i}\left(x_{i} \leq x\right)=\int_{0}^{x} f_{i} d z
$$

Using Eq. (49) gives

$$
P_{i}\left(x_{i} \leq x\right)=\int_{0}^{x} \frac{1}{\lambda_{i}} e^{-\frac{z}{\lambda_{i}}} d z=1-e^{\frac{-x}{\lambda_{i}}}
$$

Inverting Eq. (55) gives

$$
x=-\lambda_{i} \ln \left(1-P_{i}\right)
$$

Refer to Eq. (56) as the sampling function. The $P_{i}$ are uniformly distributed between 0 and 1 . Hence, random $P_{i}$ 's chosen uniformly between 0 and 1 will give a chord length distributions for material $i$ which satisfy Eq. (49). So to populate a particular realization of the problem one begins at one end of the mixing region and, using Eq. (56), samples one chunk of material $i$. Then sample one chunk of material $j$ using Eq. (56). In this alternating way, sample one material then the other until the end of the mixing region is encountered. This is sometimes referred to as the leapfrog method for generating a realization [20]. Note that the form of Eq. (56) is particular to this choice of statistics, but the process in general is true for any statistics. One simply replaces Eq. (56) with the appropriate sampling equation derived from whatever PDF's describe the problem.

To summarize: one populates a realization using the sampling function Eq. (56). One solves Eqs. (52) and (53) obtaining the solution to one statistical realization. The procedure is repeated $N$ times and the final ensemble average solution is given by 


$$
\langle\psi\rangle=\frac{1}{N} \sum_{n=1}^{N} \psi_{n}
$$

and

$$
\langle\varphi\rangle=\frac{1}{N} \sum_{n=1}^{N} \varphi_{n},
$$

where $\psi_{n}$ and $\varphi_{n}$ are the solutions to Eqs. (52) and (53) for the $n^{t h}$ realization.

\subsection{Atomic Mix - Implementation}

The atomic mix approximation is anything but new. It's ease of implementation and speed make it a popular method. Casting Eqs. (52) and (53) into the atomic mix Formulation from Sec. (4.2) gives

$$
\frac{1}{c} \frac{\partial}{\partial t}\langle\psi\rangle+\mu \frac{\partial}{\partial z}\langle\psi\rangle+\left\langle\sigma_{a}\right\rangle\langle\psi\rangle=\langle S\rangle+\frac{c\left\langle\sigma_{a}\right\rangle\langle\phi\rangle}{2},
$$

and

$$
\frac{\partial}{\partial t}\langle\phi\rangle=-c\left\langle\sigma_{a}\right\rangle\langle\phi\rangle+\left\langle\sigma_{a}\right\rangle \int\langle\psi\rangle d \vec{\Omega} .
$$

Again, this no-correlation approximation is crude and assumes that the average two stochastic quantities can be factored as $\langle\sigma \psi\rangle=\langle\sigma\rangle\langle\psi\rangle$, for example. This method was included not because it is new, but because it is easy and popular - for comparison with the new methods. Because the atomic mix formulation is relatively easy and fast to implement and run, it is important to know when it works. If it works there may not be any need to 
perform a more complicated analysis.

\subsection{Standard Model - Implementation}

Now adapt the Standard Model formulation for the stochastic solution of Eqs. (52) and (53). How does one do this? Recall from Sec. (4.3) that the Standard Model is equivalent to choosing the closure relation Eq. (37) to approximate the statistical coupling terms that arise in the restricted ensemble averaging of the transport equation. Recall also that these coupling terms arise because the restricted averaging operator does not commute with the gradient operator in the transport equation. Because Eq. (53) contains no spacial derivatives, the restricted ensemble averaging of Eq. (53) will not give rise to a new coupling term. So to adapt the Standard model to Eqs. (52) and (53) simply make the assumption that the original closure relation Eq. (37) still holds. So restricted ensemble averaging of Eqs. (52) and (53), and using the Standard Model closure relation gives

$$
\frac{1}{c} \frac{\partial}{\partial t}\left(p_{i} \psi_{i}\right)+\mu \frac{\partial}{\partial z}\left(p_{i} \psi_{i}\right)+\sigma_{a i}\left(p_{i} \psi_{i}\right)=\frac{c \sigma_{a i}}{2}\left(p_{i} \phi_{i}\right)+\frac{|\mu|}{\lambda_{j}}\left(p_{j} \Psi_{j}\right)-\frac{|\mu|}{\lambda_{i}}\left(p_{i} \Psi_{i}\right)
$$

and

$$
\frac{\partial}{\partial t}\left(p_{i} \phi_{i}\right)=-c \sigma_{a i}\left(p_{i} \phi_{i}\right)+\sigma_{a i} \int_{-1}^{1}\left(p_{i} \psi_{i}\right) d \mu
$$

with $i, j=1,2$ and $i \neq j$. This represents a set of four coupled differential equations for the restricted ensemble averages $\psi_{i}$ and $\varphi_{i}$. The the ensemble average solution is given by 


$$
\langle\psi\rangle=p_{1} \psi_{1}+p_{2} \Psi_{2}
$$

and

$$
\langle\varphi\rangle=p_{1} \varphi_{1}+p_{2} \varphi_{2}
$$

just as in the Standard Model.

The closure relation used to derive this model is exact for transport in a purely absorbing media in 1-D slab geometry described by HM statistics. That is the case here. But now one also has the equation for material temperature included. In deriving Eqs. (61) and (62) it is assumed that the closure relation for the Standard Model still holds. But in fact, as will be seen in the next section, it is no longer exact when the material temperature is included.

\subsection{Su-pomraning - Implementation}

The closure relation Eq. (37) is only approximate when there is scattering present. This was the motivation behind the new closure proposed by Su and Pomraning and which was described in Sec. (4.4), i.e. to account for the effects of scattering in a stochastic setting. Here, there is no scattering. But there is coupling to a material temperature. And this coupling to material temperature can be viewed as a kind of effective scattering. It is for this reason that the closure used in the Standard Model is no longer exact when material temperature is included.

\subsubsection{Material Temperature Coupling seen as an Effective Scatterer}

Just how the coupling to material temperature can be seen as a kind of effective scattering can be easily shown. While Eqs. (52) and (53) cannot in general be solved analytically, 
they decouple approximately by time integrating the temperature equation over a small interval $\Delta t=\left(t-t_{0}\right)>0$. Integrating Eq. (53) gives

$$
\phi(t, z)=\phi\left(t_{0}, z\right) e^{-\sigma_{a} c \Delta t}+\sigma_{a} e^{-\sigma_{a} c t} \int_{t_{0}}^{t} e^{\sigma_{a} c t^{\prime}} I\left(t^{\prime}, z\right) d t^{\prime}
$$

where $I(t, z)=\int_{-1}^{1} \psi(t, z, \mu) d \mu$. Now approximate the time integral in Eq. (65) using the trapezoid quadrature rule getting

$$
\phi(t, z) \cong \phi\left(t_{0}, z\right) e^{-\sigma_{a} c \Delta t}+\frac{\sigma_{a} \Delta t}{2}\left[I(t, z)+e^{-\sigma_{a} c \Delta t} I\left(t_{0}, z\right)\right] .
$$

Substituting the right hand side of Eq. (66) into Eq. (52) gives

$$
\frac{1}{c} \frac{\partial}{\partial t} \psi(t, z, \mu)+\mu \frac{\partial}{\partial z} \psi(t, z, \mu)+\sigma_{a} \psi(t, z, \mu)=S_{E F F}+\frac{\sigma_{s E F F}}{2} \int_{-1}^{1} \psi\left(t, z, \mu^{\prime}\right) d \mu^{\prime},(\text { EQ 67) }
$$

where

$$
S_{E F F}(z, t)=\frac{\sigma_{a} c}{2} e^{-\sigma_{a} c \Delta t}\left[\phi\left(t_{0}, z\right)+\frac{\sigma_{a} \Delta t}{2} I\left(t_{0}, z\right)\right],
$$

is an effective source and

$$
\sigma_{s E F F}=\frac{\sigma_{a}^{2} c \Delta t}{2}
$$

is an effective scattering coefficient. It is important to note that even though the scattering coefficient is zero $\left(\sigma_{s}=0\right)$, the coupling to material temperature can be thought of as 
introducing an effective source and effective isotropic scattering. This suggests that in order to treat the statistical nature of the coupling in Eqs. (52) and (53) one must first be able to treat the statistical effects of scattering in the transport equation itself, even though there is no real scattering present. Therefore, in an effort to improve upon the Standard Model when applied to Eqs. (52) and (53), adapt the Su and Pomraning closure relation by allowing the effective scattering coefficient given by Eq. (69) to take the place of real scattering.

\subsubsection{The Su-Pomraning Closure with the Effective Scatterer}

Recall from Sec. (4.4) that the model proposed by Su and Pomraning has the same general form as the Standard Model but introduces a new general closure, Eq. (38). In the 1-D slab geometry this closure has the form,

$$
\left\langle\psi \nabla \chi_{i}\right\rangle=\kappa|\mu|\left[\frac{\left(p_{j} \psi_{j}\right)}{\lambda_{j}}-\frac{\left(p_{i} \psi_{i}\right)}{\lambda_{i}}\right]+\frac{\eta|\mu|}{2}\left[\frac{\int\left(p_{i} \psi_{i}\right) d \mu^{\prime}}{\lambda_{i}}-\frac{\int\left(p_{j} \psi_{j}\right) d \mu^{\prime}}{\lambda_{j}}\right]
$$

with two proposed formulas for the weighting parameters $\kappa$ and $\eta$. The 1-D slab form of this model (in a pure absorber) is then given by the four coupled differential equations

$$
\frac{1}{c} \frac{\partial}{\partial t}\left(p_{i} \psi_{i}\right)+\mu \frac{\partial}{\partial z}\left(p_{i} \psi_{i}\right)+\sigma_{a i}\left(p_{i} \psi_{i}\right)=\frac{c \sigma_{a i}}{2}\left(p_{i} \phi_{i}\right)+\left\langle\psi \nabla \chi_{i}\right\rangle
$$

and

$$
\frac{\partial}{\partial t}\left(p_{i} \phi_{i}\right)=-c \sigma_{a i}\left(p_{i} \phi_{i}\right)+\sigma_{a i} \int_{-1}^{1}\left(p_{i} \psi_{i}\right) d \mu
$$

with $i, j=1,2$ and $i \neq j$, and Eq. (70) used for the closure term. 
Here, because the material is a pure absorber, $\sigma_{s}=0$, and Eqs. (70) through (72) reduce to the Standard Model. However make the direct substitution of $\sigma_{s E F F}$ for $\sigma_{s}$ in the expressions for $\kappa$ and $\eta$ gives something new. The hope is that the coupling term Eq. (70) will capture the statistical effects of material temperature coupling in exactly the same way it was meant to capture the statistical effects of scattering.

Consider the first set of relations proposed for $\kappa$ and $\eta$, namely Eqs. (41) and (42). Applying these to Eq. (67) gives the weighting parameters

$$
\kappa=\frac{\sqrt{\left\langle\sigma_{a}\right\rangle \mid\left\langle\sigma_{a E F F}\right\rangle} \mid\left[\left\langle\sigma_{a E F F}\right\rangle\left(\sigma_{a 2}-\sigma_{a 1}\right)^{2}+\left\langle\sigma_{a}\right\rangle\left(\sigma_{a E F F 2}-\sigma_{a E F F 1}\right)^{2}\right]}{\left[\left\langle\sigma_{a E F F}\right\rangle\left(\sigma_{a 2}-\sigma_{a 1}\right)\right]^{2}+\left[\left\langle\sigma_{a}\right\rangle\left(\sigma_{a E F F 2}-\sigma_{a E F F 1}\right)\right]^{2}}
$$

and

$$
\eta=0
$$

where

$$
\sigma_{a E F F i}=\sigma_{a i}-\sigma_{s E F F i}=\sigma_{a i}\left(1-\frac{\sigma_{a i} c \Delta t}{2}\right)
$$

and

$$
\left\langle\sigma_{a E F F}\right\rangle=\left\langle\sigma_{a}\right\rangle-\left\langle\sigma_{s E F F}\right\rangle
$$

The quantity $\sigma_{a E F F i}$ can be thought of as a reduced effective absorption coefficient which is allowed to be negative - hence the introduction of the absolute value in Eq. (73). 
Refer to the closure given by Eqs. (70), (73), and (74) as variation 1.

Using the second expression for $\kappa$ and $\eta$ proposed by Su and Pomraning, Eqs. (45) and (46), gives variation number 2. Again, applying to Eq. (67) gives

$$
\eta=\frac{1}{\sqrt{\left|1-\frac{\left\langle\sigma_{s E F F}\right\rangle}{\left\langle\sigma_{a}\right\rangle}\right|}}
$$

and

$$
\kappa=\frac{\frac{\left\langle\sigma_{s E F F}\right\rangle}{\langle\sigma\rangle}}{\sqrt{1-\frac{\left\langle\sigma_{s E F F}\right\rangle}{\left\langle\sigma_{a}\right\rangle} \mid}}
$$

The whole point of these two variations is to include the effects of scattering in the stochastic solution. Here the idea of material temperature viewed as an effective isotropic scattering is being used within this same framework to account for the statistical effects of temperature coupling. Physically this is a reasonable hope, in that both scattering and material temperature serves to redistribute photons to other directions of travel. Scattering does it via a scattering event, and material temperature coupling does it via a heating and thermal emission. But the net result is in both cases isotropic redistribution of photons.

\subsection{Numerical Results}

In Sec. (8.0) were presented several methods for approximating the stochastic solution to the 1-d slab problem with HM statistics. Here are present the numerical solutions to the various methods discussed in Sec. (8.0). 


\subsection{Numerical Techniques}

All methods are solved using diamond difference for spatial-discretization of the gradient operator and implicit time-discretization for the temperature and specific intensity time derivatives - see Appendix B or reference [17]. The angular variable $\mu$ is treated by standard discrete ordinate methods - again see Appendix B or reference [17]. At every time step, the temperature and specific intensity were updated to the same time stamp via an inner (fixed point) iteration, of fixed length. The maximum size $\Delta z$ of the grid spacing was chosen to guarantee positivity of the radiation specific intensity according to

$$
\Delta z=\frac{|\mu|_{\min }}{\left(\sigma_{\max }+\frac{1}{c \Delta t}\right)},
$$

where $|\mu|_{\min }$ is the smallest absolute value from the S-16 quadrature ordinates, $\sigma_{\max }$ is the maximum of the two material absorption coefficients [17]. The time step $\Delta t$ is chosen to be fixed at the value $\Delta t=1.0 e-12$ seconds. This choice of time step is somewhat arbitrary. It was simply chosen to be of the same order of magnitude as the time it takes light to cross a typical spatial zone. Essentially a time step was used which was suitable for an explicit time step method but in an implicit method. This was done so that the problem would run without complication. It was a very conservative choice but it worked without difficulty.

The benchmark averages were computed using 1024 deterministic realizations. This number was arrived at by trial and error. Solutions were generated for various numbers of realizations and determined that 1024 sufficient by observing the convergent behavior of 
the solution as the number of realizations was increased. The error bars on the benchmark curves are within the thickness of the lines used to plot the graphs.

\subsection{Numerical Stability of Source Iteration}

A comment is in order regarding the way in which the radiation transport equation was solved at each time step. It was solved using a standard technique commonly called Source Iteration [17]. In this method, any integral terms are approximated by estimating a value for the solution and using the estimate to evaluate the integral term. The equation is solved, and the integral terms are again updated using the new solution. This process is repeated until convergence. In Appendix (A) it is shown that Source Iteration applied to Eqs. (70) and (71) has the stability criteria that

$$
\max \left[\frac{\sigma_{s 1}}{\sigma_{1}}, \frac{\sigma_{s 2}}{\sigma_{2}}, \frac{\eta}{\kappa}\right]<1
$$

In a purely absorbing media this reduces to the condition

$$
\frac{\eta}{\kappa}<1 \text {. }
$$

This is guaranteed to be true in a purely absorbing media with the original expressions for $\eta$ and $\kappa$ as proposed by Su and Pomraning. But using Eqs. (77) and (78), the value $\frac{\eta}{\kappa}$ can now be greater than 1 . This means that this model, the one referred as the Su-Pomraning second variation (the one with $\eta \neq 0$ ), may not be stable under Source Iteration when $\sigma_{S E F F}$ is used to compute $\eta$ and $\kappa$. 


\subsection{Results - Constant Opacities}

Table (1) contains the data used to compute the curves shown in Figs. (6) through (13). Notice that the values of the volume ratio for material $i$ at any given point are equal to the probability of finding that material there, i.e. $V_{i}=P_{i}=\frac{\lambda_{i}}{\left(\lambda_{1}+\lambda_{2}\right)}$. The different $\lambda_{i}$ were specifically chosen so that the volume ratios were the same for all the test runs. Hence the results for the atomic mix model will be the same for all test runs. This allows us to more clearly see the effect of changing stochastic structure, not just volume ratio, on the answer.

Each of the figures (6) through (13) have four curves. Curve (A) is the benchmark value. Curve (B) is the value returned by the atomic mix model, Eqs. (59) and (60). Curve (C) is the result from the standard model adaptation given by Eqs. (61) and (62). Curve (D) is the result from the adaptation of the Su-Pomraning model, Eqs. (70) through (72), with the effective scattering used in calculation of the weighting factors via Eqs. (73) and (74), i.e, variation number 1 . When calculating the weighting factors using Eqs. (77) and (78), i.e. variation number 2 , the source iteration technique for solution of the transport equation was not stable, and would not converge. It is easily seen why this is so. Using the values in Table (1) to compute the stability criteria Eq. (81) for variation number 2 gives

$$
\frac{\eta}{\kappa}=\frac{\left\langle\sigma_{s E F F}\right\rangle}{\left\langle\sigma_{a}\right\rangle}=10.0181
$$

Since this number must be less than one for the method to be stable, it clearly is not stable here. Hence, no results for this method were calculated. 
Figures (6) through (9) are plots of $\frac{\langle\varphi\rangle}{a T_{o}^{4}}$ versus time step as computed for the benchmark calculation and the three models which converged under source iteration. In other words, the time evolution of the ensemble average of the material temperature energy density scaled to the initial material temperature $T_{o}$, as computed at the outgoing edge of the mixing region, as shown in Fig. (5).

Figures (10) through (13) are plots of $\frac{\langle\text { Trans }\rangle}{c a T_{o}^{4}}$ versus time step as computed for the three different models and the benchmark, where

$$
\text { Trans }=\int_{0}^{1}(\psi(t, z=L, \mu)) \mu d \mu
$$

In other words, it shows the time evolution of the ensemble average of the radiant energy streaming out the outgoing edge of the mixing region scaled to the initial material temperature $T_{o}$

Figures (14) through (17) are plots of the variance in the benchmark for material temperature energy density and radiation specific intensity as scaled by the answer for each time step. In other words it is plots of percentage variance given by $\frac{\sqrt{\left\langle\varphi^{2}\right\rangle-\langle\varphi\rangle^{2}}}{\langle\varphi\rangle}$ and $\frac{\sqrt{\left\langle(\text { Trans })^{2}\right\rangle-\langle\text { Trans }\rangle^{2}}}{\langle\text { Trans }\rangle}$ as a function of time step. The time step axis for the variances is on a $\log$ scale. 
Table 1.Parameters for 1-D Slab - Constant Opacities

\begin{tabular}{|c|c|c|}
\hline \multicolumn{3}{|c|}{$\sigma_{a 1}=1000 / \mathrm{cm} \quad \sigma_{a 2}=5 / \mathrm{cm}$} \\
$\psi(t, z=0, \mu)=0.3 \mathrm{KeV}$ \\
$T_{o}=0.03 \mathrm{KeV}$ \\
\multicolumn{3}{|c|}{$L=0.15 \mathrm{~cm}$} \\
\hline Figure \# & $\lambda_{1}(\mathbf{c m})$ & $\lambda_{2}(\mathrm{~cm})$ \\
\hline \hline $6,10,14$ & $5.0 \mathrm{e}-3$ & $5.0 \mathrm{e}-1$ \\
\hline $7,11,15$ & $5.0 \mathrm{e}-4$ & $5.0 \mathrm{e}-2$ \\
\hline $8,12,16$ & $5.0 \mathrm{e}-5$ & $5.0 \mathrm{e}-3$ \\
\hline $9,13,17$ & $5.0 \mathrm{e}-6$ & $5.0 \mathrm{e}-4$ \\
\hline
\end{tabular}

FIGURE 6. $\langle\varphi\rangle$ versus Time Step - Constant opacities

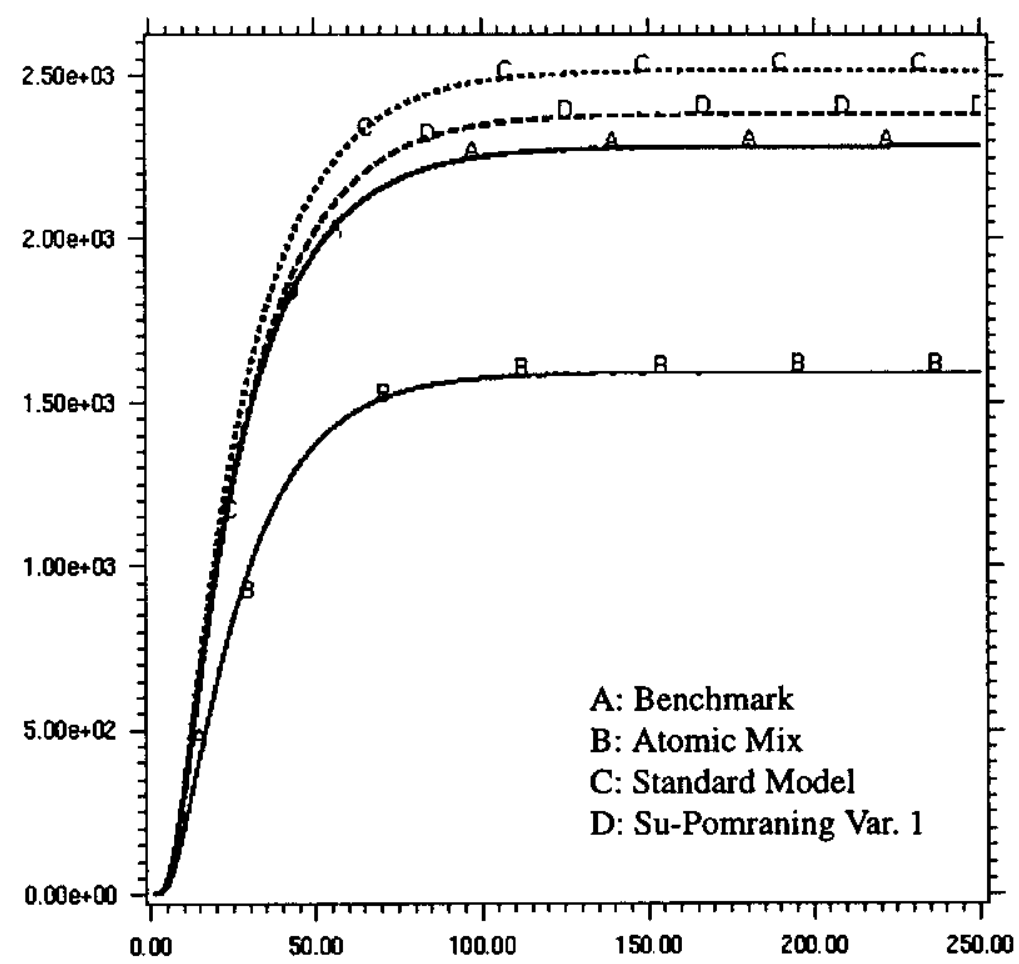


FIGURE 7. $\langle\varphi\rangle$ versus Time Step - Constant opacities

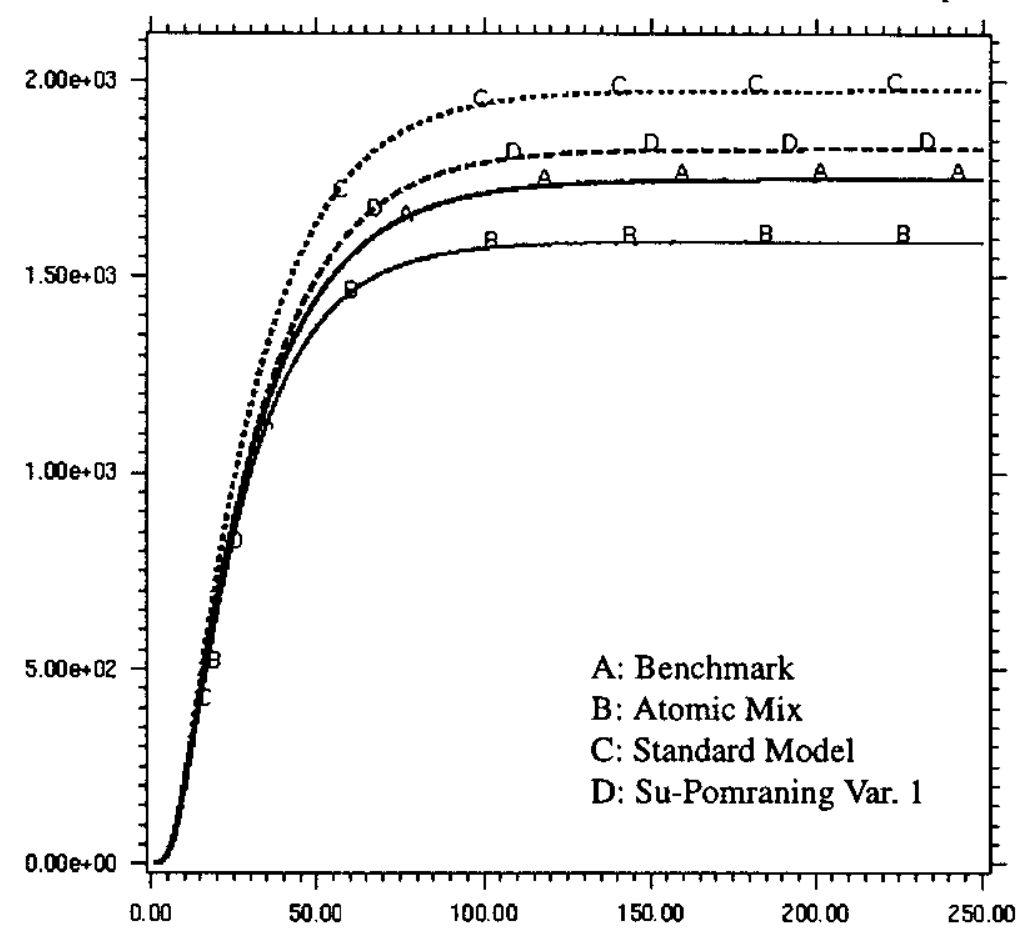

FIGURE 8. $\langle\varphi\rangle$ versus Time Step - Constant Opacities

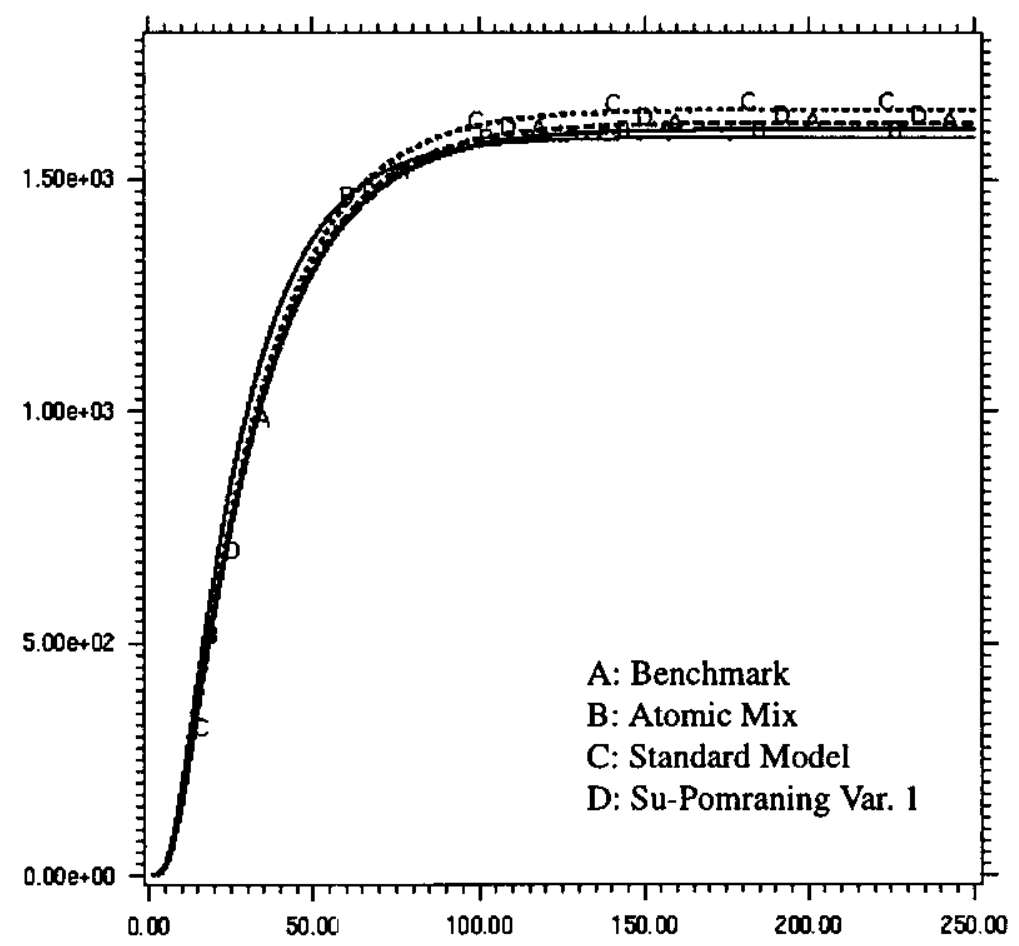


FIGURE 9. $\langle\varphi\rangle$ versus Time Step - Constant Opacities

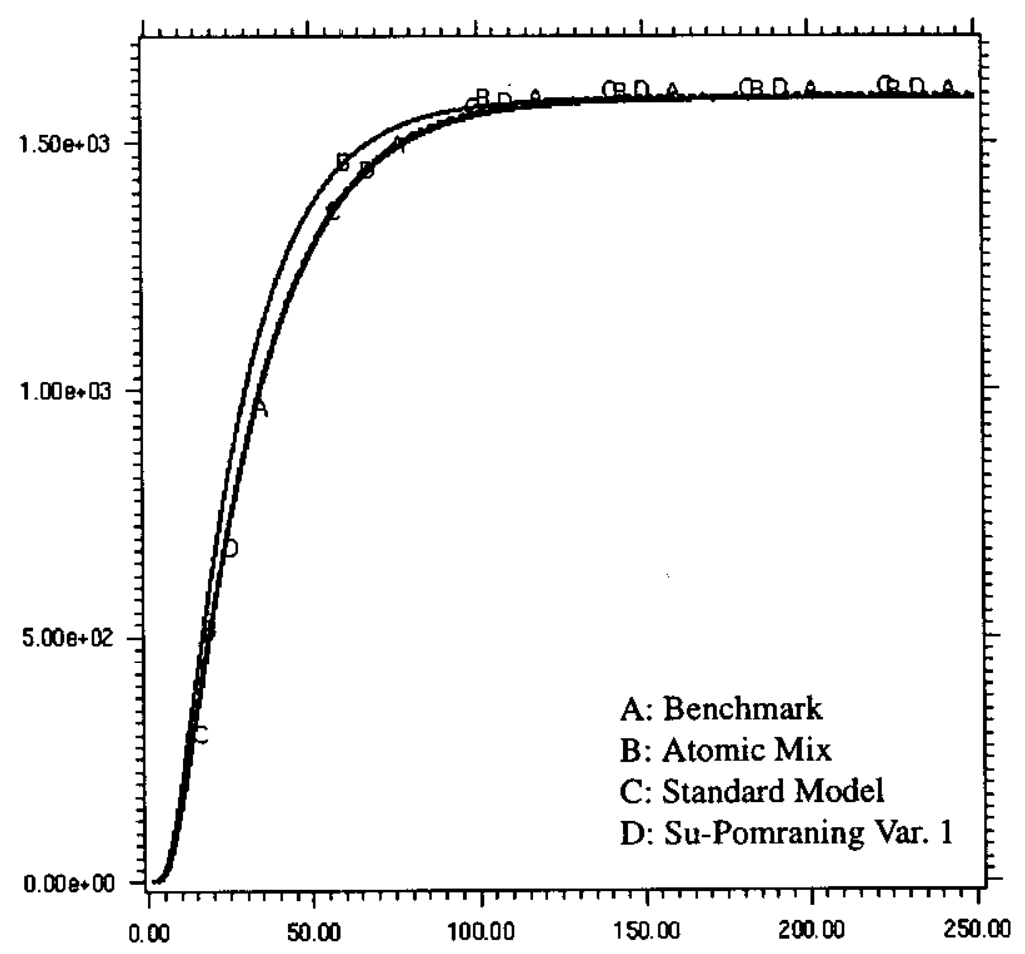

FIGURE 10. 〈Trans $\rangle$ versus Time Step - Constant Opacities

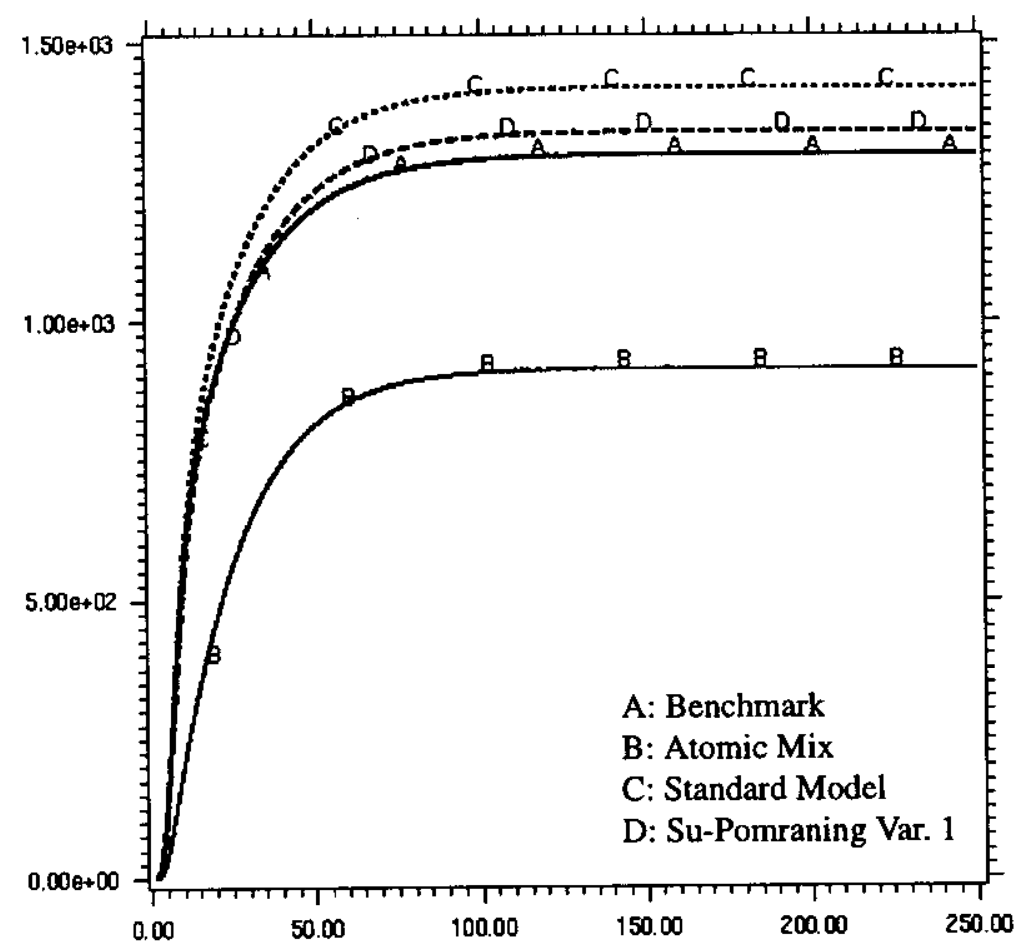


FIGURE 11. $\langle$ Trans $\rangle$ versus Time Step - Constant Opacities

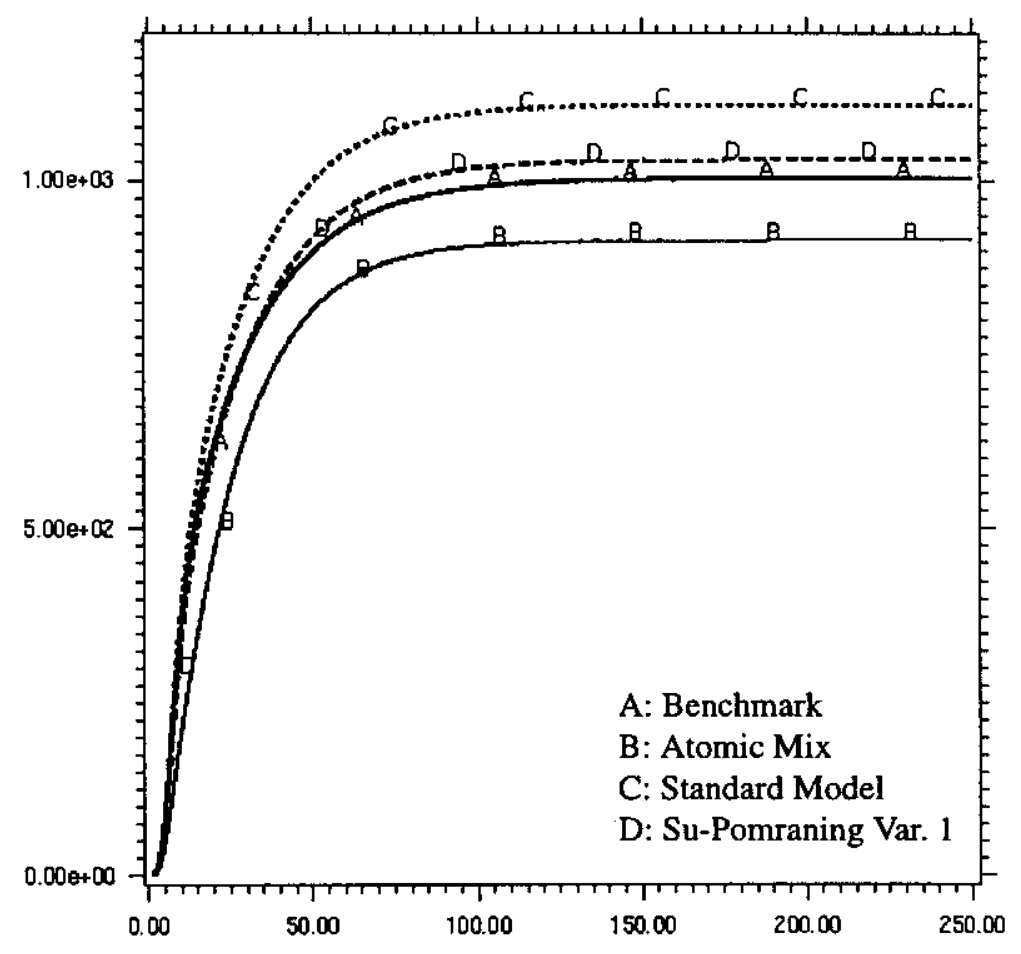

FIGURE 12. $\langle$ Trans $\rangle$ versus Time Step - Constant Opacities

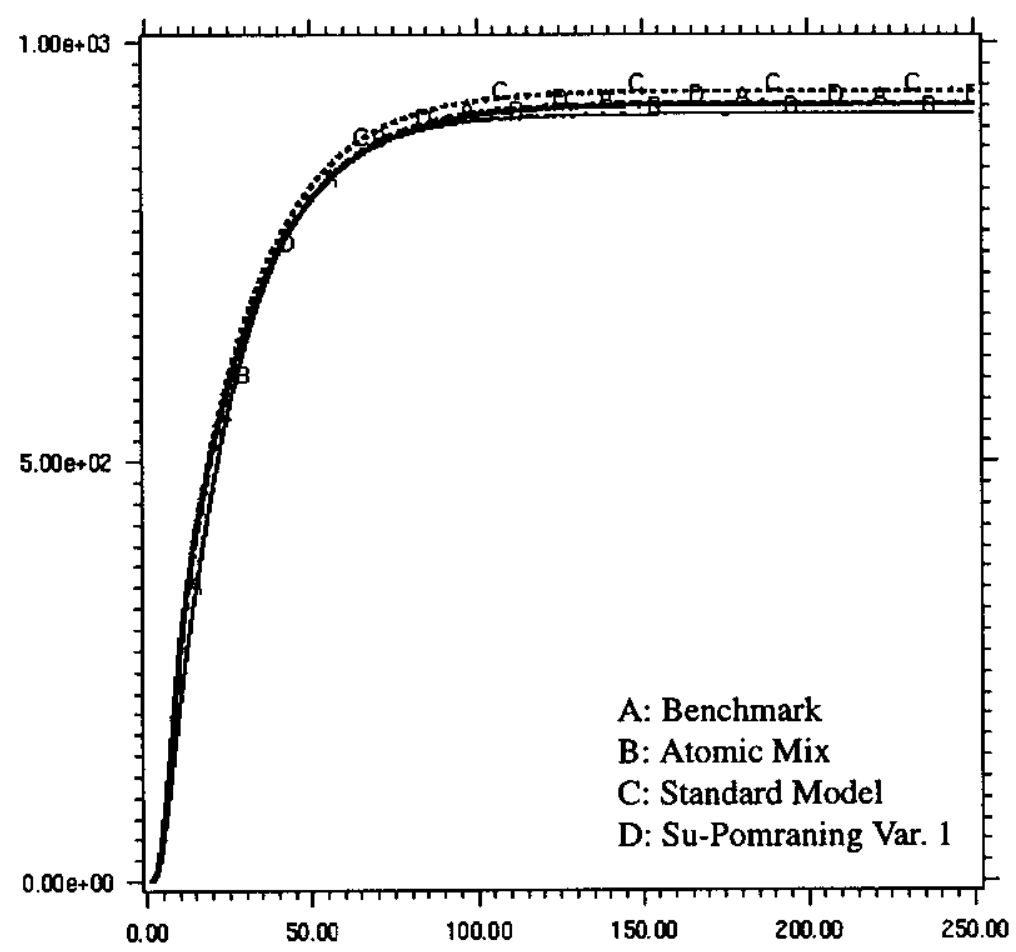


FIGURE 13. 〈Trans $\rangle$ versus Time Step - Constant Opacities

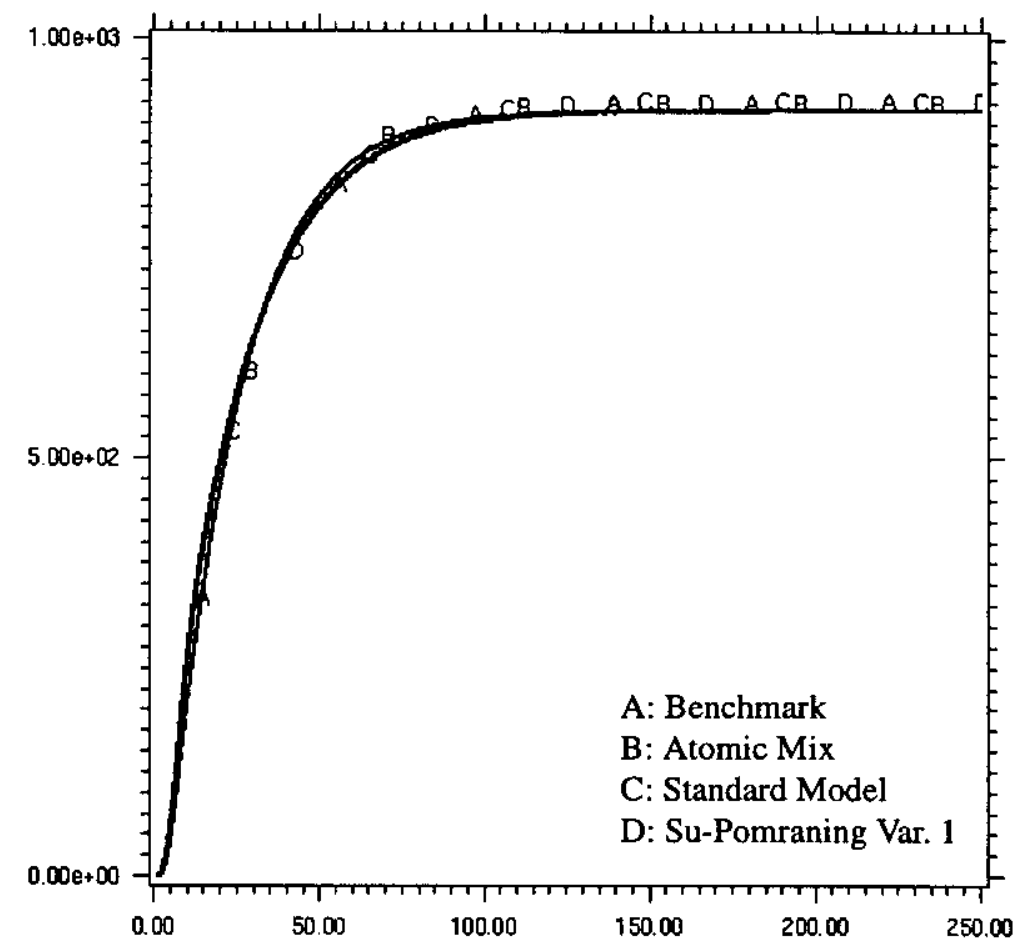

FIGURE 14. Benchmark Variance - Constant Opacities

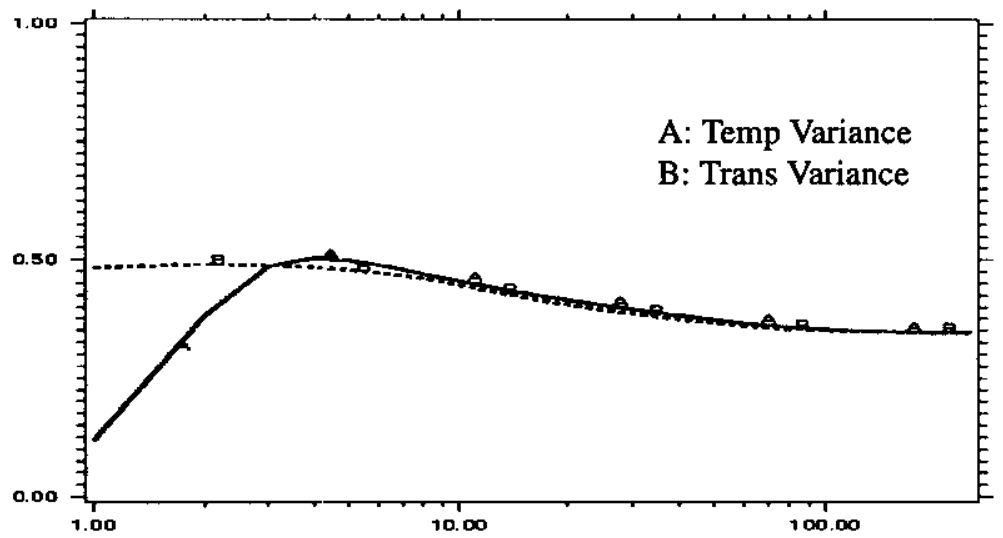


FIGURE 15. Benchmark Variance - Constant Opacities

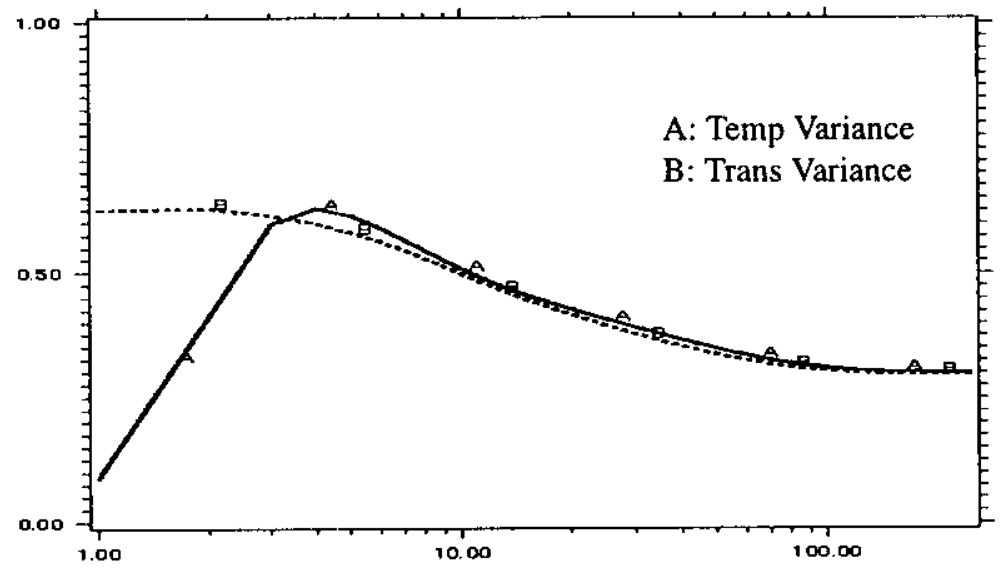

FIGURE 16. Benchmark Variance - Constant Opacities

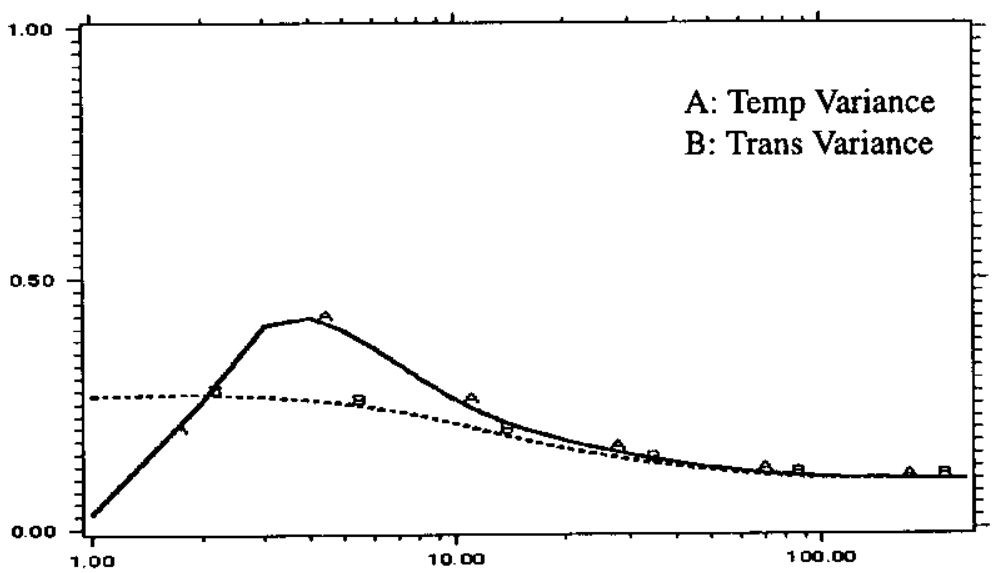

FIGURE 17. Benchmark Variance - Constant Opacities

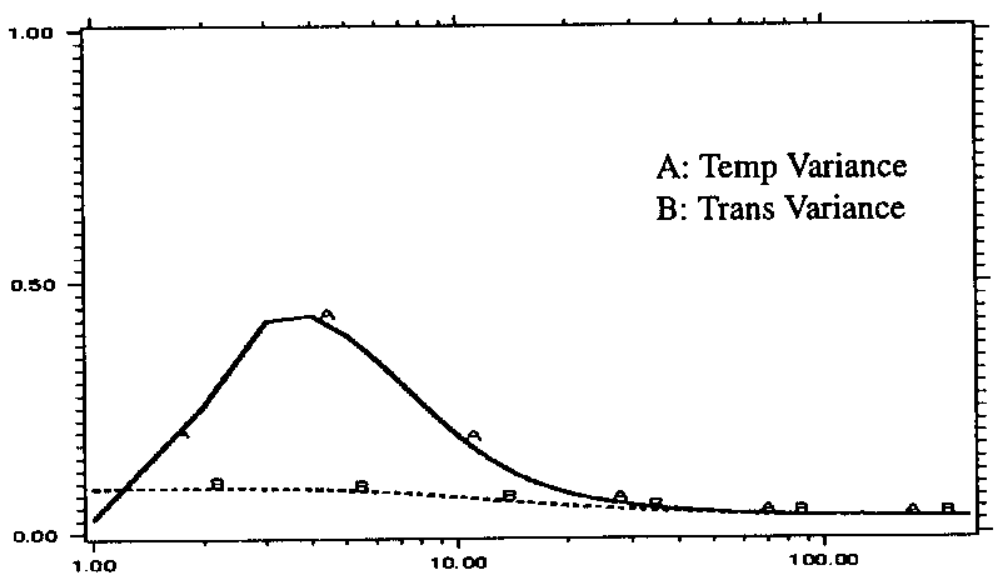


In all of the resulting curves the atomic mix model (B) consistently underestimates the steady state values of radiation transmission and material temperature. This demonstrates the well known effect that the atomic mix assumption overestimates the absorption of photons [11]. Also notice the general trend that the simple application of the Standard Model (C) consistently overestimates both the material temperature and the transmission of radiation. In treating the coupling to material temperature as a kind of effective scattering in the Su-Pomraning model (D), there is consistent improvement over both atomic mix and the Standard Model.

Special notice should be taken of the behavior of the three approximate models in the limit of small correlation length, i.e as the $\lambda_{i}$ get small. While curves (C) and (D) everywhere converge to the benchmark answer (A), the atomic mix value (B) only does so in the steady state. This was surprising. Analysis of the models show that the coupled equations for the specific intensity as given by Eqs. (61) and (71) go to the atomic mix equation (59) as the $\lambda_{i}$ go to zero. However the material temperature equations (62) and (72) do not go to the atomic mix equation for material temperature Eq. (60), except in the steady state. But the coupled equation models both converge numerically to the benchmark answer as the $\lambda_{i}$ get small. So let us take a closer look at the atomic mix equation for material temperature, Eq. (60). Consider the material temperature equation without the coupling to the radiation intensity

$$
\frac{\partial \phi}{\partial t}=-c \sigma \phi
$$


The exact answer for $\langle\phi\rangle$ on a binary stochastic media is

$$
\langle\phi\rangle=\phi_{o}\left[p_{1} e^{-\sigma_{1} c t}+p_{2} e^{-\sigma_{2} c t}\right]
$$

But the atomic mix assumption would give

$$
\langle\phi\rangle=\phi_{o} e^{-\left(p_{1} \sigma_{1}+p_{2} \sigma_{2}\right) c t}
$$

where $\phi_{o}=a T_{o}^{4}$. They are not the same and no amount of letting $\lambda_{i}$ go to zero will make them so. In fact the $\lambda_{i}$ do not appear explicitly at all. This leads to the conclusion that there is something missing from the "obvious" formulation of the atomic mix model for the material temperature. The simple atomic mix formulation does not get the correct transient behavior in the small chunk limit. Evidently it is not sufficient to simply use averaged material properties in the small chunk limit. Yet neither is it yet clear what the correct interpretation of atomic mix should be to get the correct time dependent behavior for the atomic mix material temperature. Because the temperature and the radiation intensity are coupled in Eqs. (59) and (60) one might expect that this effect will influence the atomic mix average value for the transmission as well, which is indeed apparent in Fig. (13).

The variance on all of the runs was at or below $50 \%$ with the highest values being during the transient phase. This means that for this problem the ensemble average might be a good representation of the answer on an individual statistical realization, with the lowest fit during the transient phase. 
If the equations were in fact approaching the atomic mix answer as the chunks were made smaller, all of the correlations would go to zero and the variances would also go to zero. Looking at the behavior of the variances it is obvious that the models are in fact doing this in the steady state. But in the transient the correlations are not going to zero. This is yet another manifestation of the fact that the atomic mix assumption is not correct for the transient, even in the small correlation length limit.

\subsection{Results - Temperature Dependent Opacities}

All of the numerical results so far in the 1-D slab geometry have been computed on various models with the opacities being held constant. However real problems are not generally this accommodating. Letting the opacities be dependent upon the material temperature, and hence time variable, introduces a whole new level of complication to the problem. To investigate how well these 1-D slab models perform with temperature dependent opacities, the opacities of the two materials were allowed to depend on temperature as

$$
\sigma_{a i}=\frac{C_{i}}{T^{3.5}}
$$

where $C_{i}$ is some arbitrary constant chosen for material $i$. How does this change the level of approximation in our models? Consider the restricted ensemble average of the product $\left\langle\chi_{i} \sigma_{a} \psi\right\rangle$. With constant opacities this average factors as

$$
\left\langle\chi_{i} \sigma_{a} \psi\right\rangle=p_{i} \sigma_{a i} \psi_{i}
$$

The derivation of the models in this chapter (except for the benchmark of course) depend 
on Eq. (88) being true. But with temperature dependent opacities Eq. (88) no longer holds.

So how well do the models work with material temperature dependent opacities? All physical parameters other than the opacities were kept the same as in the constant opacity runs and are shown again in Table (2).

Table 2.Parameters for 1-D Slab - Variable Opacities

\begin{tabular}{|c|c|c|}
\hline \multicolumn{3}{|c|}{$\psi(t, z=0, \mu)=0.3 \mathrm{KeV}$} \\
$T_{o}=0.03 \mathrm{KeV}$ \\
$L=0.15 \mathrm{~cm}$ \\
\hline Figure \# & $\lambda_{1}(\mathrm{~cm})$ & $\lambda_{2}(\mathrm{~cm})$ \\
\hline \hline $18,22,26$ & $5.0 \mathrm{e}-3$ & $5.0 \mathrm{e}-1$ \\
\hline $19,23,27$ & $5.0 \mathrm{e}-4$ & $5.0 \mathrm{e}-2$ \\
\hline $20,24,28$ & $5.0 \mathrm{e}-5$ & $5.0 \mathrm{e}-3$ \\
\hline $21,25,29$ & $5.0 \mathrm{e}-6$ & $5.0 \mathrm{e}-4$ \\
\hline
\end{tabular}

The opacities are variable according to Eq. (87) with $C_{1}=1.07 \times 10^{28}$ and $C_{2}=1.73 \times 10^{22}$. These choices together with Eq. (87) are based on the assumption that the absorption opacities arise via an inverse-Bremsstrahlung process for a plasma of two materials with the electrons of each material in thermodynamic equilibrium. The two materials chosen were gold for material 1 and hydrogen for material 2 . To handle the extremes of the temperature ranges, a minimum of $0.1 / \mathrm{cm}$ and a maximum of $10000 / \mathrm{cm}$ were imposed on the opacities. They were allowed to vary within this range according to Eq. (87). The data of these runs is shown in Figs. (18) through (25). The layout of the plots, and the quantities plotted are identical to that in the previous section - only now the opacities are temperature dependent. 
Just as in the previous constant-opacity runs, Figs. (18) through (21) are plots of $\frac{\left\langle T^{4}\right\rangle}{T_{o}^{4}}$ versus time step as computed for the benchmark calculation and the three models which converged under source iteration. Figures (22) through (25) are plots of $\frac{\langle\operatorname{Trans}\rangle}{c a T_{o}^{4}}$ versus time step as computed for the three different models and the benchmark. Figures (26) through (29) are plots of the scaled benchmark variances $\frac{\sqrt{\left\langle\varphi^{2}\right\rangle-\langle\varphi\rangle^{2}}}{\langle\varphi\rangle}$ and $\frac{\sqrt{\left\langle(\operatorname{Trans})^{2}\right\rangle-\langle\text { Trans }\rangle^{2}}}{\langle\text { Trans }\rangle}$ as a function of time step. The time step axis for the variances is again on a log scale.

FIGURE 18. $\langle\varphi\rangle$ versus Time Step - Variable Opacities

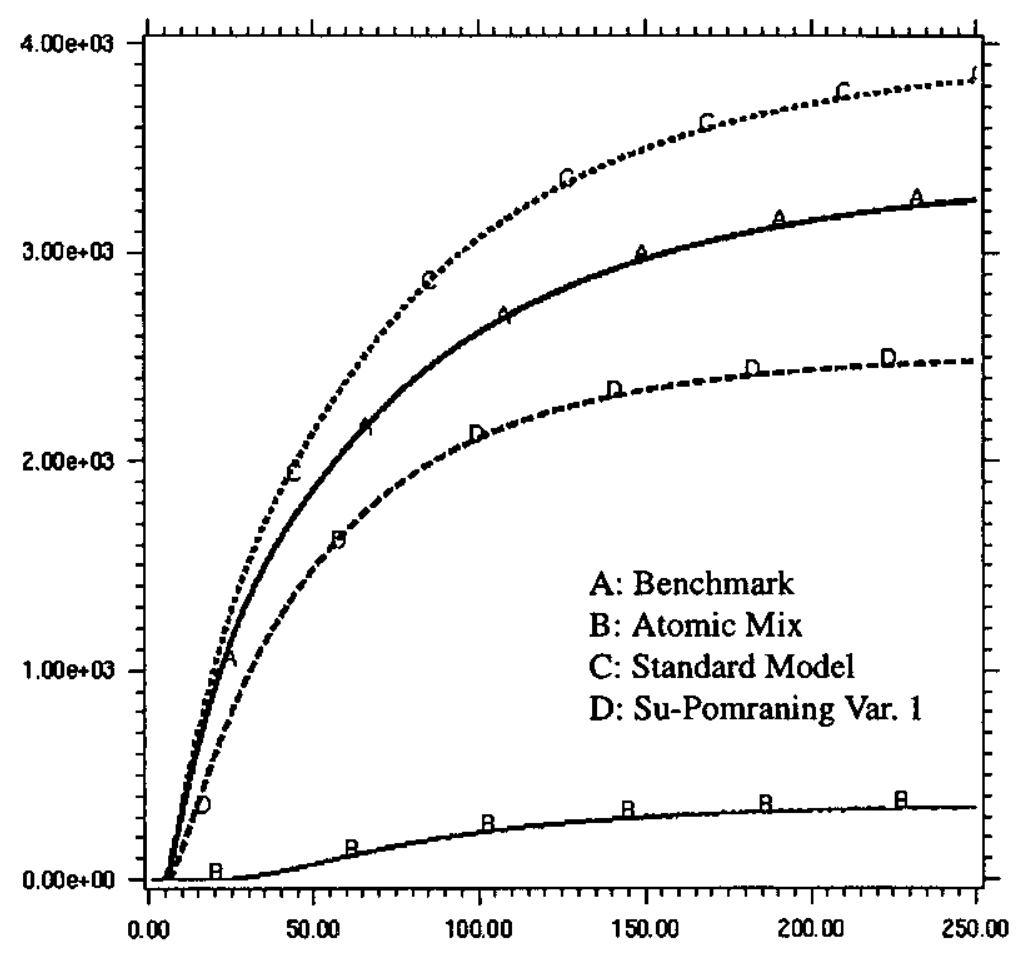


FIGURE 19. $\langle\varphi\rangle$ versus Time Step - Variable Opacities

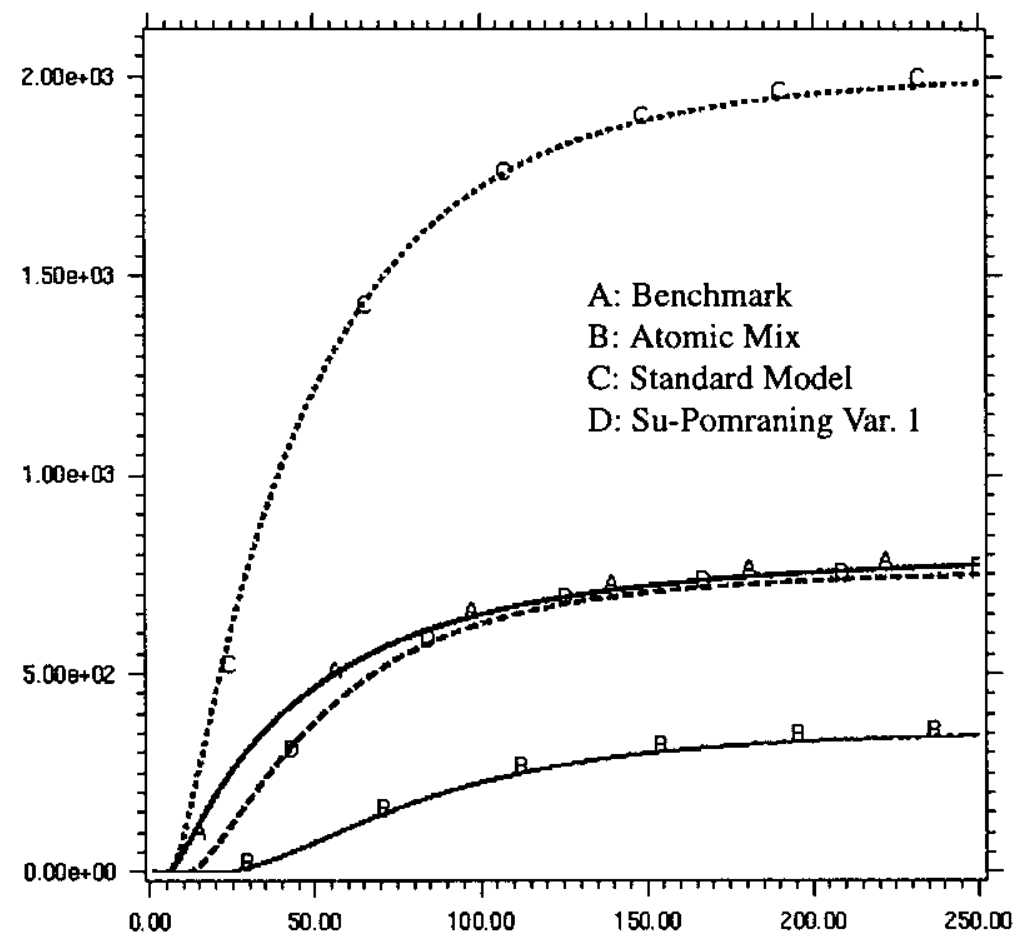

FIGURE 20. $\langle\varphi\rangle$ versus Time Step - Variable Opacities

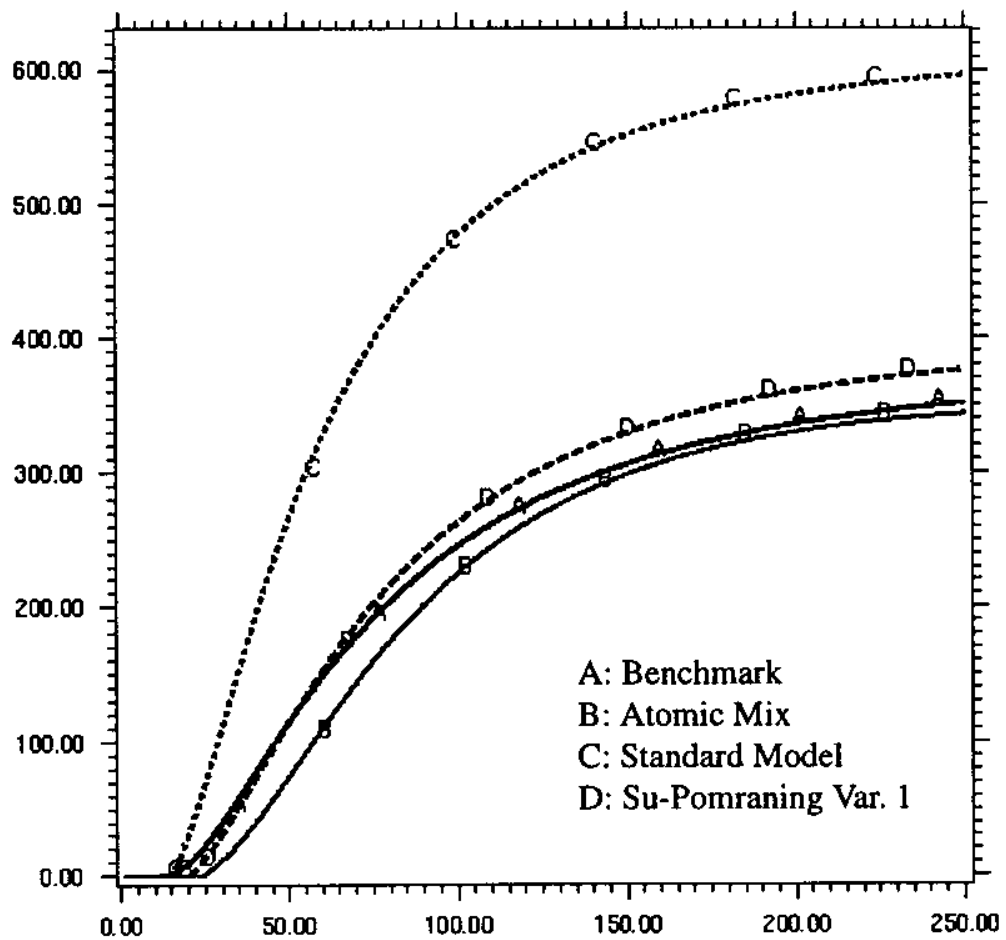


FIGURE 21. $\langle\varphi\rangle$ versus Time Step - Variable Opacities

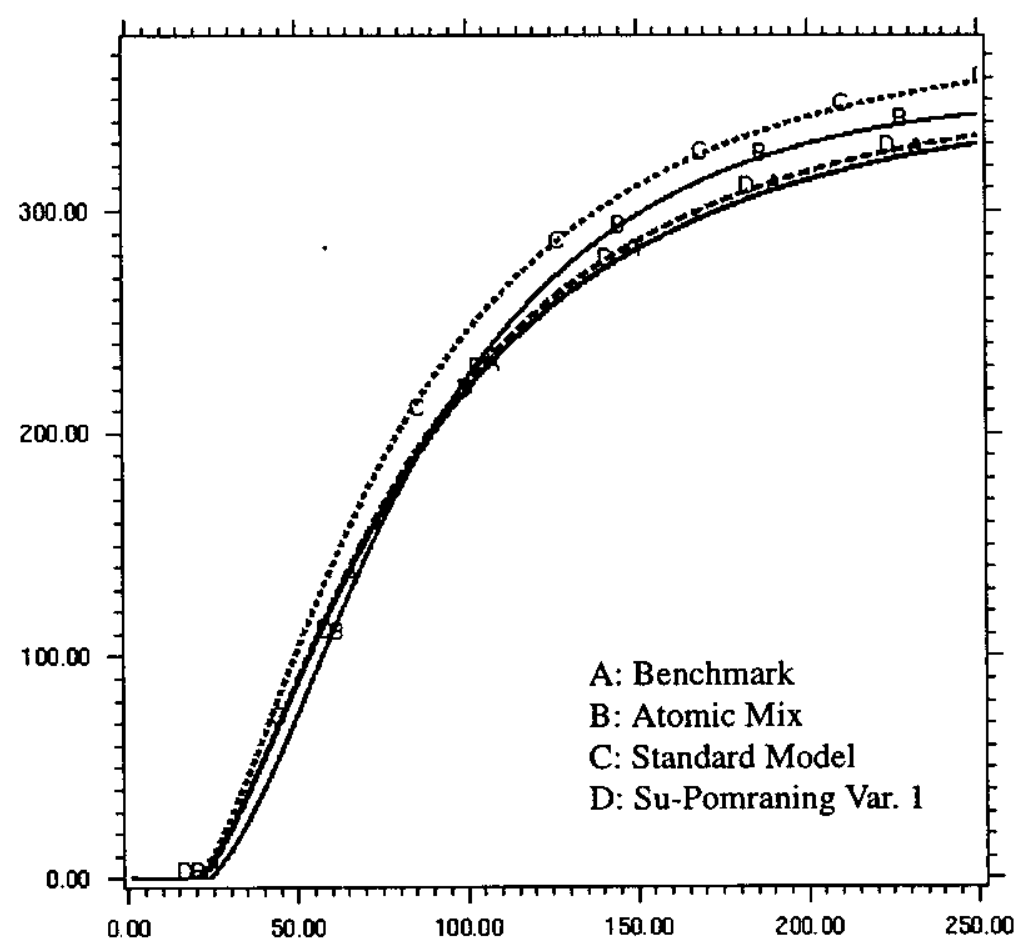

FIGURE 22. 〈Trans $\rangle$ versus Time Step - Variable Opacities

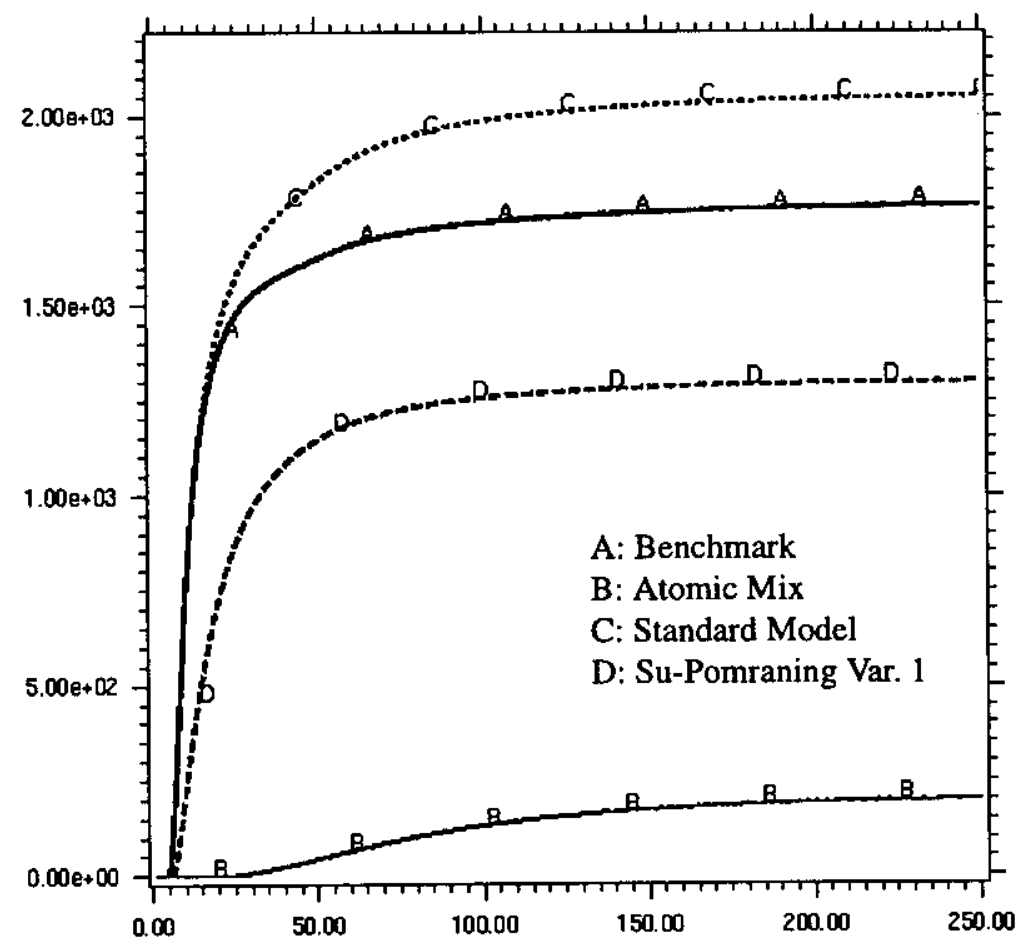


FIGURE 23. 〈Trans $\rangle$ versus Time Step - Variable Opacities

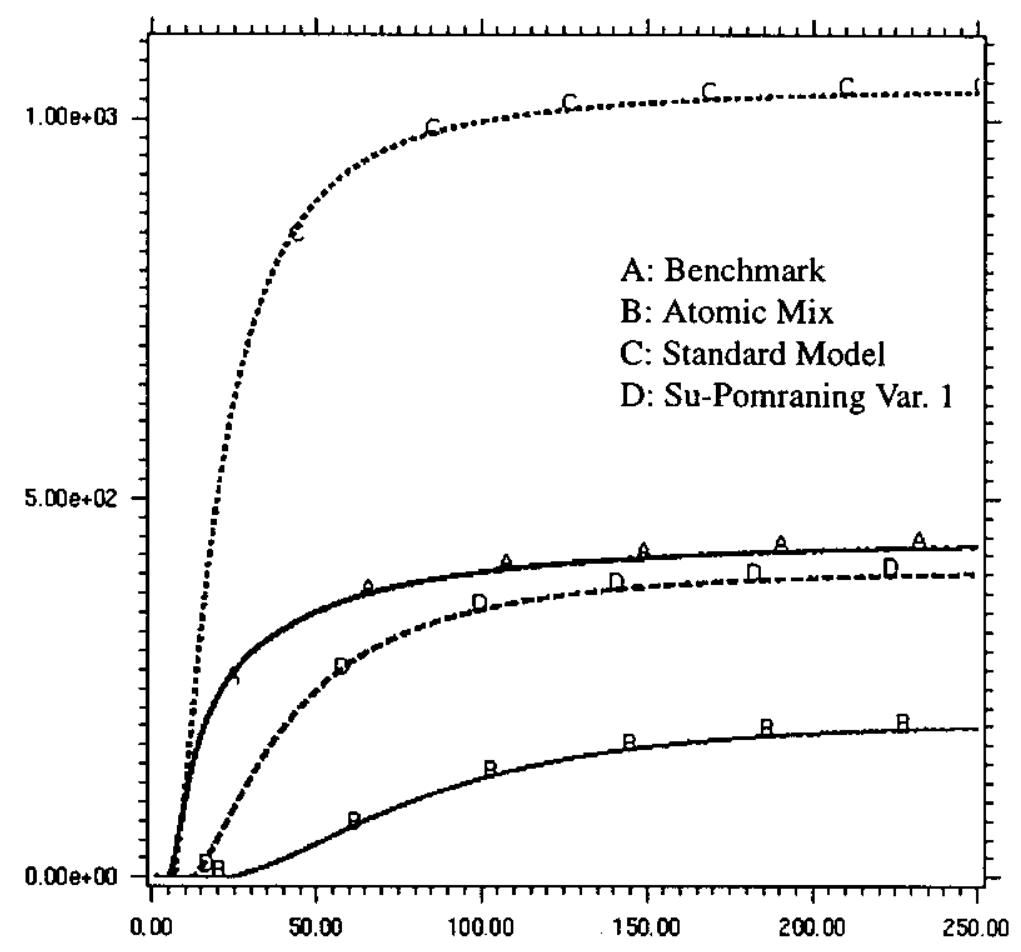

FIGURE 24. 〈Trans〉 versus Time Step - Variable Opacities

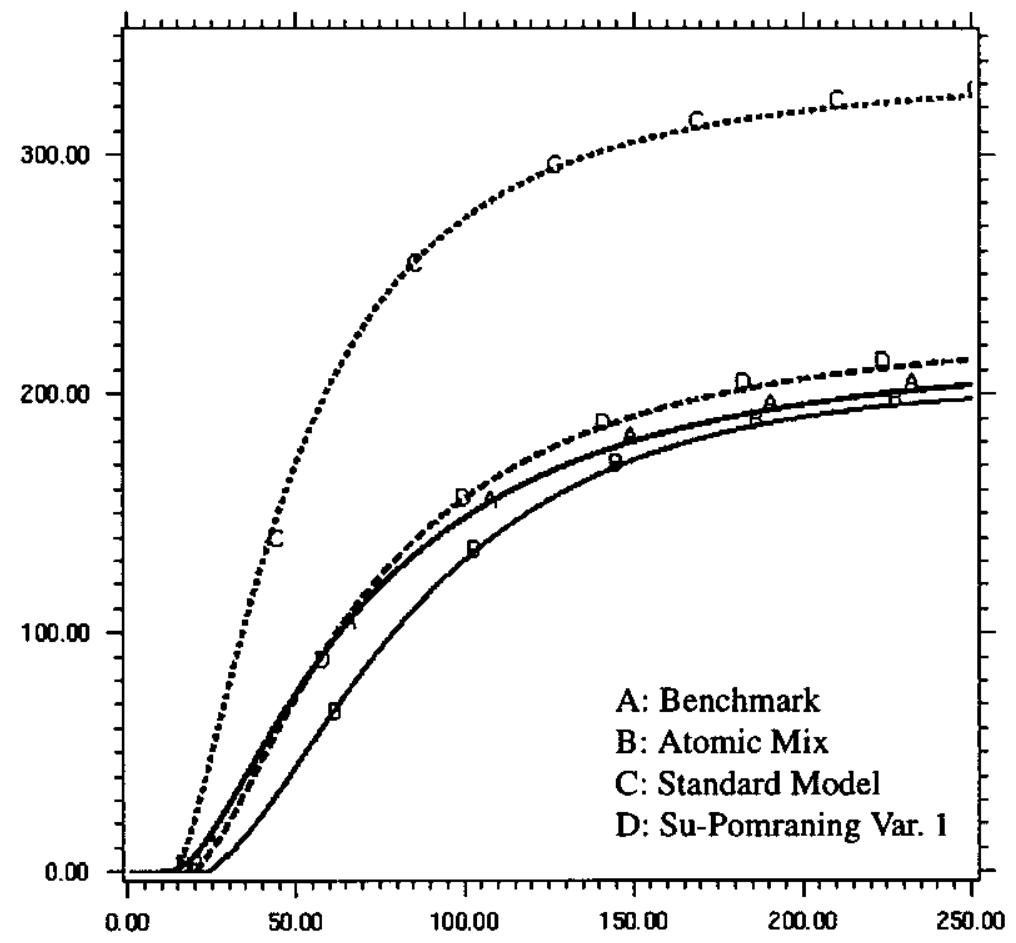


FIGURE 25. 〈Trans $\rangle$ versus Time Step - Variable Opacities

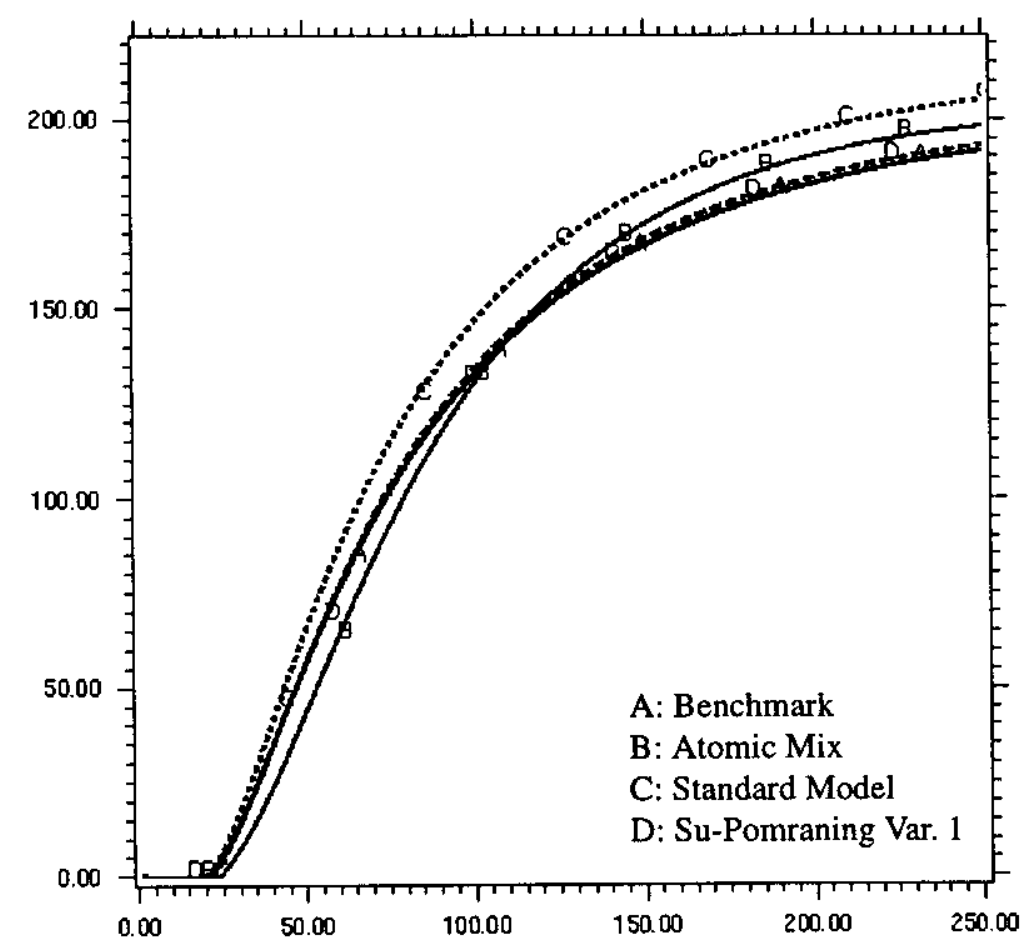

FIGURE 26. Benchmark Variance - Variable Opacities

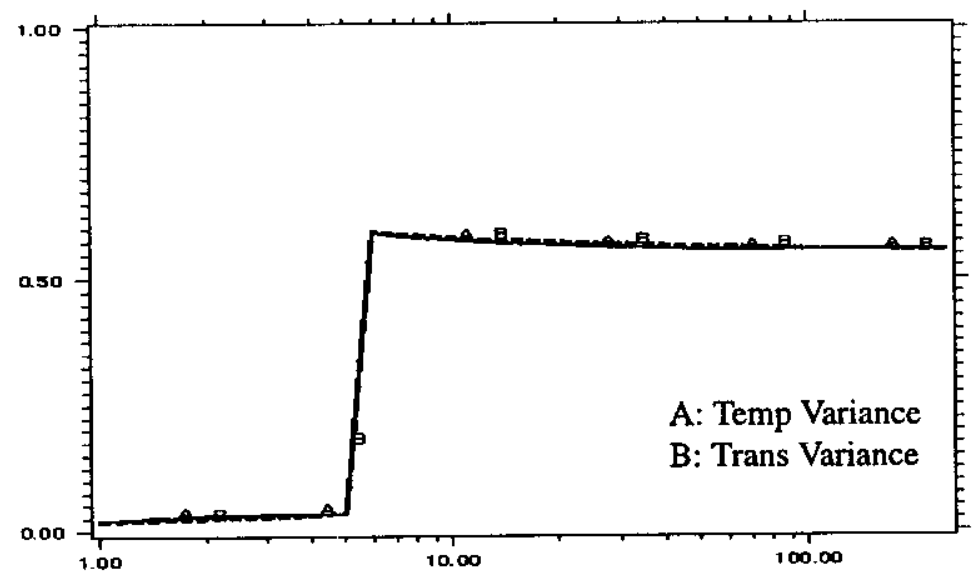


FIGURE 27. Benchmark Variance - Variable Opacities

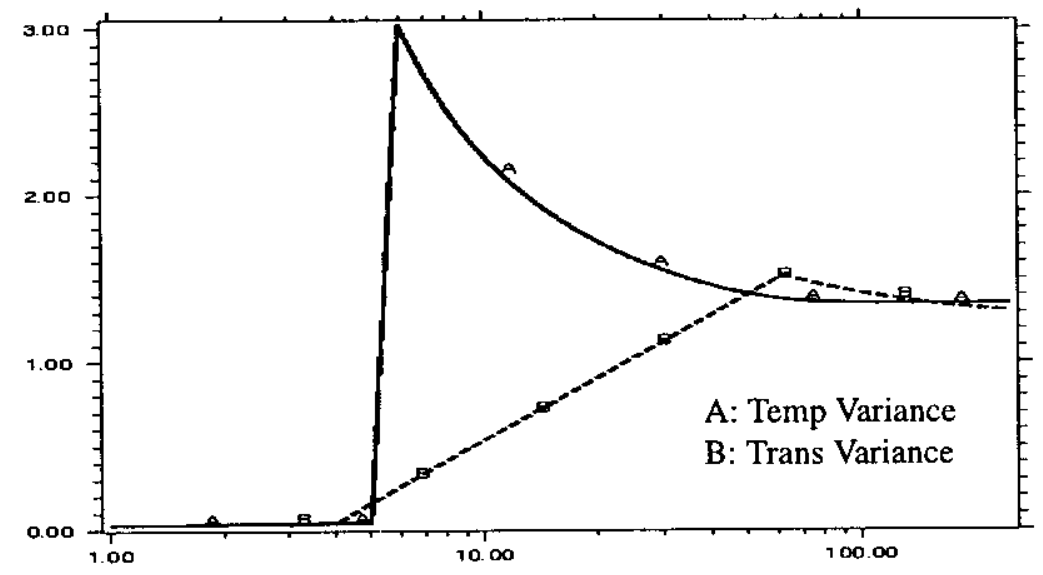

FIGURE 28. Benchmark Variance - Variable Opacities

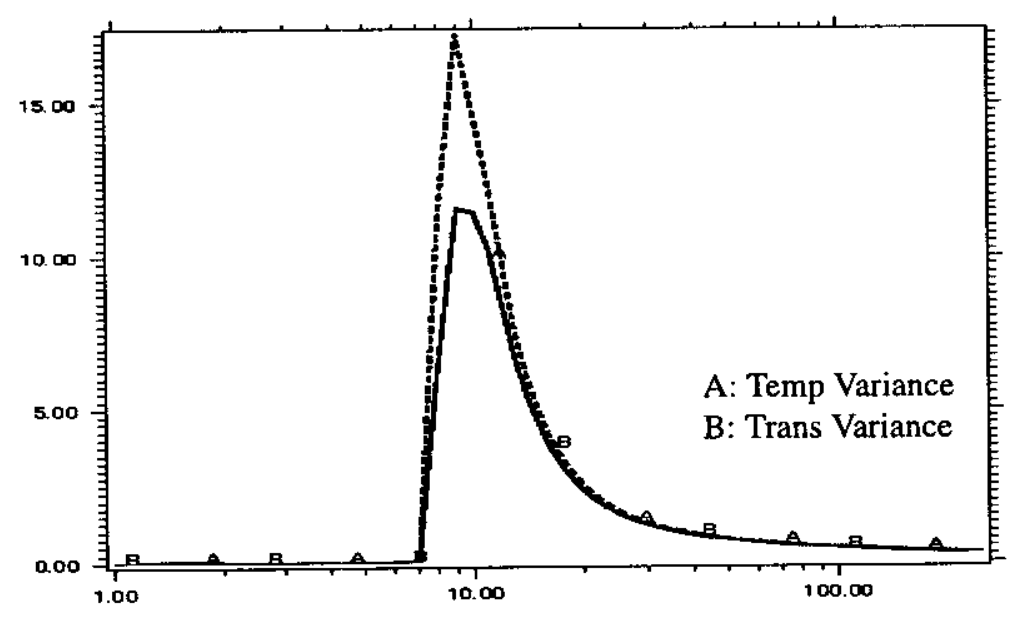

FIGURE 29. Benchmark Variance - Variable Opacities

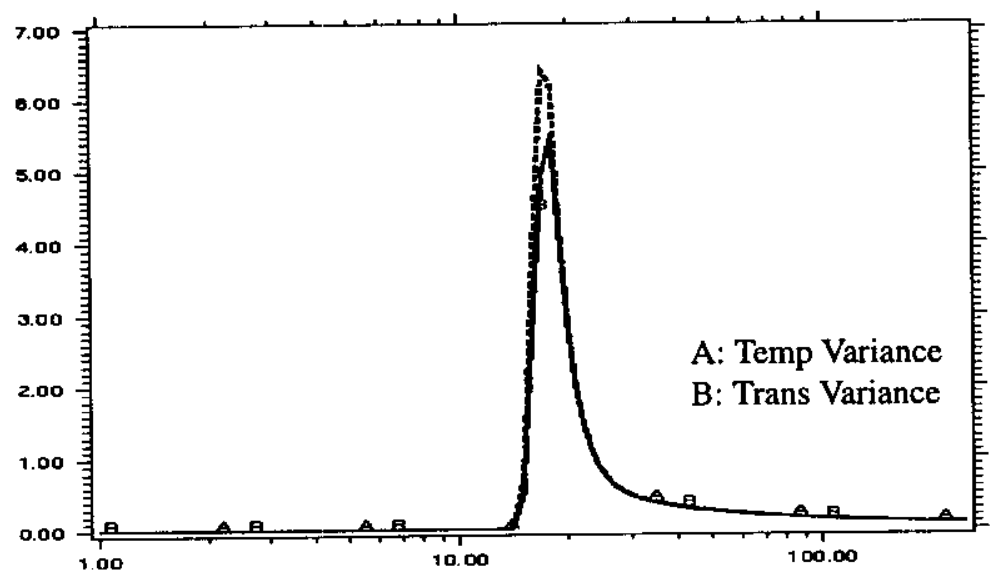


Clearly the various approximate models do not do nearly as well when the opacities are strong functions of the material temperature. The general trend of all models converging to the same answer as the chunk sizes are refined is still present, as it should be. The general over absorption of the atomic mix model is also still present as is the error in the transient of the atomic mix model. However the Su-Pomraning variation using temperature as an effective scatterer is in this case not significantly better than the Standard Model except in a small space of chunk sizes. Evidently the approximation introduced via the assumption that Eq. (88) still holds is a significant source of error. And any model which hopes to accomplish the modeling of real temperature dependent systems is going to have to better treat the new statistical correlations that material temperature coupling introduces.

Notice how that the variances in the transient period have grown dramatically with the introduction of temperature dependent opacities. Obviously there are very large variations between different statistical realizations. This calls into question the value of the ensemble averages for representing individual experimental measurements on this kind of problem. A meaningful comparison could only be made with averages taken over a large number of experimental measurements, covering a large range of statistical samples for this problem.

Once again it is clear that the models are recovering the atomic mix answer in the steady state, but not in the transient. If it were, the correlations and hence the variance would go to zero in the transient. And clearly this is not happening. 


\section{STOCHASTIC MODELS IN 1-D SPHERICAL GEOMETRY}

This chapter proposes some new methods for modeling the stochastic solution to Eqs. (10) and (11) in a 1-D spherical geometry. These are essentially the 1-D spherical formulations of the same methods developed in the chapter on 1-D slab geometry. The formulations of these different models are presented in Sec. (11.0) and the numerical solution results in Sec. (12.0). First restrict the problem to be a purely absorbing media. Then in Sec. (12.4) this restriction is relaxed by allowing the opacities to be temperature dependent. In this geometry, the mixing region is composed of alternating shells of the two materials surrounding a homogenous core which acts as an isotropic radiation source at a constant temperature, as shown in Fig. (30).

FIGURE 30. Typical 1-D Spherical Geometry Media

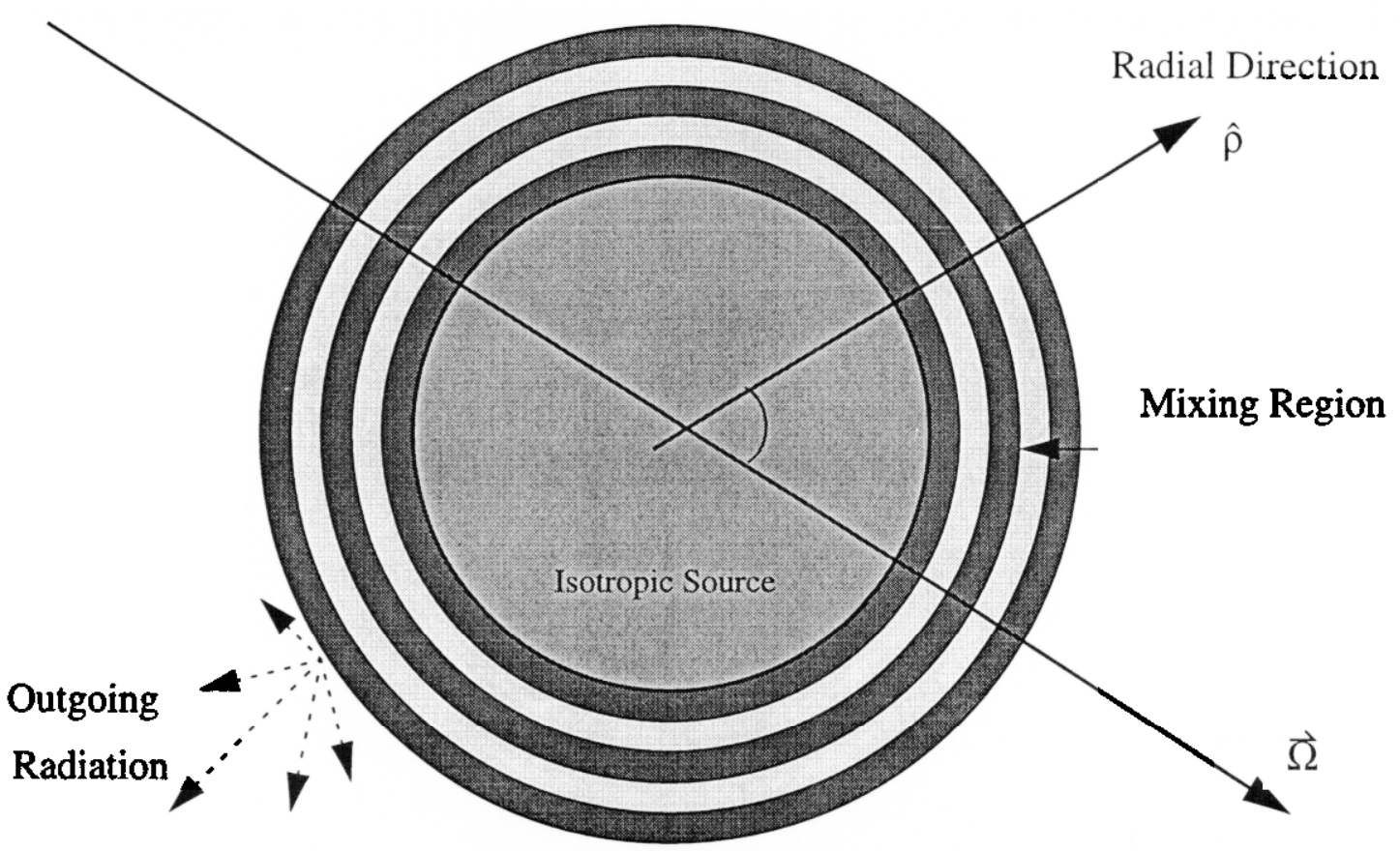




\subsection{Markov Approximation in a 1-D Sphere}

The mixing region is described by alternating spherical shells of random thickness with Poisson PDF functions along the radial direction. The PDF's for the chord lengths (read shell width) of material $i$ along the radial direction are assumed to given by

$$
f_{i}(\rho)=\frac{1}{\lambda_{i}} e^{-\frac{\rho}{\lambda_{i}}},
$$

where $\lambda_{i}$ is then the average width of a shell of material $i$ along the radial direction. Then the probability of finding material $i$ at any given point is still given by

$$
p_{i}=\frac{\lambda_{i}}{\left(\lambda_{1}+\lambda_{2}\right)}
$$

just as in the slab geometry case. Let $\mu$ be the cosine of the angle between the ray $\vec{\Omega}$ and the radial direction $\hat{\rho}$. Consider the probability of transition from one shell to the next when taking a step from $\vec{l}$ to $\vec{l}+d \vec{\gamma}$ along the ray $\vec{\Omega}$. In the case of homogeneous statistics this probability is independent of position. Here however this is not the case. This is because for a shell of fixed thickness along the radial direction, the thickness as seen by a photon traveling across the shell at an angle $\vec{\Omega} \cdot \hat{\rho}$ will depend not only upon angle but also upon radius. The thickness seen by a photon at a given angle is now dependent upon it's position within the problem. This is easily seen. Consider Fig. (31). 
FIGURE 31.

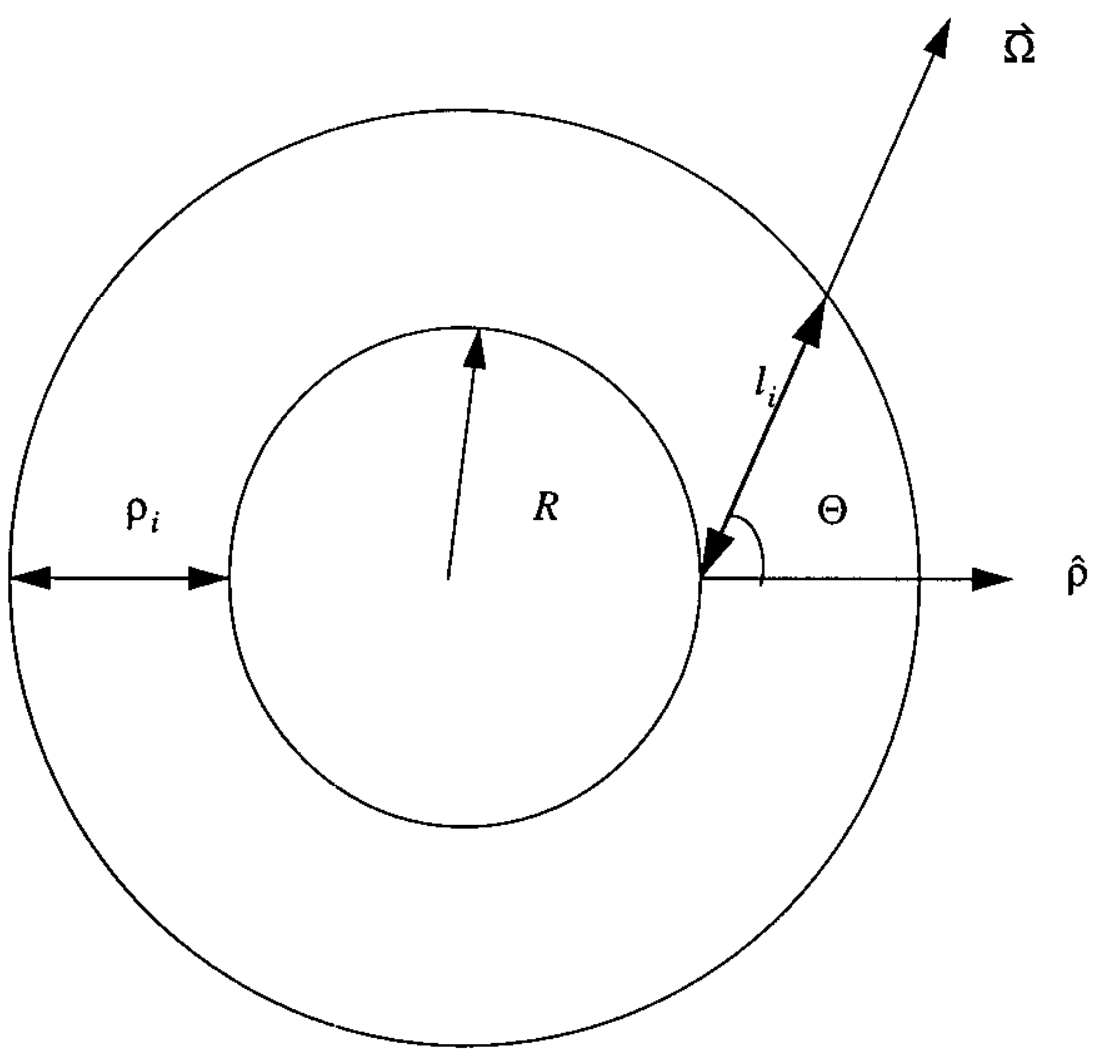

Let the width of a shell of material $i$ as seen along the ray $\vec{\Omega}$ and at radius $R$ be given by $l_{i}$ with the thickness of the same shell seen along the radial direction as $\rho_{i}$. Let the ray $\bar{\Omega}$ make an angle $\Theta$ with the radial direction $\hat{\rho}$. Then simple geometry gives

$$
l_{i}(R, \Theta)=-R \cos \Theta+\sqrt{\left(R+\rho_{i}\right)^{2}-R^{2} \sin ^{2} \Theta}
$$

Notice that in the limit as the radius gets much bigger than the thickness of the shell, i.e, $\frac{\rho_{i}}{R} \ll 1$, Eq. (91) becomes 


$$
l_{i}=\frac{\rho_{i}}{\cos \Theta}
$$

which is the slab geometry result Eq. (50). However, as the radius of the problem grows, the geometry of the sphere does not necessarily just become a 1-D curved slab problem. This is because radiation from one side of the core can be radiated across the sphere to the other side - a geometric connectivity missing in the 1-D slab geometry. But, when the only sources of radiation are in the core, this cross-problem connectivity disappears if there is no scattering or material temperature coupling present. Also, if one specifies a core boundary condition such that the radiation field is constant there, then this geometric connectivity disappears regardless of the presence of any scattering or temperature coupling.

\subsection{Formulation of the Different Methods}

Again, one wishes to obtain the stochastic solution, i.e the ensemble averages of the radiation specific intensity $\langle\psi\rangle$ and the material temperature energy density $\langle\varphi\rangle$, in the 1-D spherical media shown in Fig. (30) and described by Eqs. (89) and (90). In a purely absorbing 1-D spherical geometry, Eqs. (10) and (11) become

$$
\frac{1}{c} \frac{\partial \psi}{\partial t}+\mu \frac{\partial \psi}{\partial \rho}+\frac{\left(1-\mu^{2}\right)}{\rho} \frac{\partial \psi}{\partial \mu}+\sigma_{a} \psi=\frac{c \sigma_{a}}{2} \phi(t, \rho)+S
$$

and

$$
\frac{\partial}{\partial t} \phi(t, \rho)=-c \sigma_{a} \phi(t, \rho)+\sigma_{a} \int_{-1}^{1} \psi d \mu
$$

where $\sigma_{a}$ is the absorption opacity. Because the materials are purely absorbing, $\sigma=\sigma_{a}$ 
and $\sigma_{s}=0$. These simplifying assumptions were made for the same reasons as in the last chapter on the 1-D slab geometry. Namely to make the problem more tractable while still retaining some of the new difficulties associated with the addition of material temperature coupling. In the slab geometry these assumptions were exactly those assumptions under which the Standard Model as presented in Sec. (4.3) is exact when material temperature coupling is absent. Here that is not quite the case. Not only are new physics introduced via the radiation coupling to material temperature but also a statistical material distribution which is not HM statistics. The goal here then is twofold. One is to see what happens with the material temperature coupling in the spherical geometry. The other is to see what effect the changing of the statistics has on the performance of the standard model and it's variations.

\subsection{Benchmark - Implementation}

The benchmark process for the 1-D spherical geometry is identical to that in the 1-D slab. The sampling functions Eq. (56) is unchanged also. Now instead of sampling the width of alternating slabs of the two materials one samples the thicknesses of alternating spherical shells. And the solutions for each realization are performed on the spherical geometry.

\subsection{Atomic Mix - Implementation}

Casting Eqs. (93) and (94) into the atomic mix formulation from Sec. (4.2) gives

$$
\frac{1}{c} \frac{\partial}{\partial t}\langle\psi\rangle+\mu \frac{\partial}{\partial \rho}\langle\psi\rangle+\frac{\left(1-\mu^{2}\right)}{\rho} \frac{\partial}{\partial \mu}\langle\psi\rangle+\left\langle\sigma_{a}\right\rangle\langle\psi\rangle=\langle S\rangle+\frac{c\left\langle\sigma_{a}\right\rangle\langle\phi\rangle}{2}
$$


and

$$
\frac{\partial}{\partial t}\langle\phi\rangle=-c\left\langle\sigma_{a}\right\rangle\langle\phi\rangle+\left\langle\sigma_{a}\right\rangle \int\langle\psi\rangle d \bar{\Omega}
$$

The level of approximation here is identical to that of the 1-D slab case in Sec. (8.2).

\subsection{Standard Model - Implementation}

Now adapt the Standard Model formulation for the stochastic solution of Eqs. (93) and (94). How? Just as in the slab geometry case, assume that the closure relation Eq. (37) is still valid. How, since Eq. (95) has a new spacial derivative term? Won't that give rise to a new coupling in the closure relation? Formally no. The distribution of material is radially symmetric. This means that the restricted ensemble average operator will commute with the angular derivative term $\frac{\partial}{\partial \mu}$. Then just as in the slab geometry case, only the $\frac{\partial}{\partial \rho}$ will give rise to a statistical coupling. So restricted ensemble averaging of Eqs. (93) and (94), and using the Standard Model closure relation gives

$$
\left[\frac{1}{c} \frac{\partial}{\partial t}+\mu \frac{\partial}{\partial \rho}+\frac{\left(1-\mu^{2}\right)}{\rho} \frac{\partial}{\partial \mu}+\sigma_{a i}\right]\left(p_{i} \Psi_{i}\right)=p_{i} S_{i}+\frac{c \sigma_{a i}}{2}\left(p_{i} \phi_{i}\right)+\frac{|\mu|}{\lambda_{j}}\left(p_{j} \psi_{j}\right)-\frac{|\mu|}{\lambda_{i}}\left(p_{i} \psi_{i}\right)
$$

and

$$
\frac{\partial}{\partial t}\left(p_{i} \phi_{i}\right)=-c \sigma_{a i}\left(p_{i} \phi_{i}\right)+\sigma_{a i} \int_{-1}^{1}\left(p_{i} \psi_{i}\right) d \mu
$$

with $i, j=1,2$ and $i \neq j$. This represents a set of four coupled differential equations for the restricted ensemble averages $\psi_{i}$ and $\varphi_{i}$. The the ensemble average solution is given 
by

$$
\langle\psi\rangle=p_{1} \psi_{1}+p_{2} \psi_{2}
$$

and

$$
\langle\varphi\rangle=p_{1} \varphi_{1}+p_{2} \varphi_{2}
$$

just as in the Standard Model. This adaptation of the standard model to my problem is identical to what was done in the 1-D slab geometry except that in the spherical geometry there is a twist. The statistics are not HM in this case. This, combined with the material to temperature coupling acting as an effective scatterer, make for two levels of approximation to explore.

\subsection{The Su-Pomraning Closure with the Effective Scatterer - Implementation}

Just as in Sec. (8.4.2) apply the Su-Pomraning closure using the effective scatterer Eq. (69). Once again this closure has the form,

$$
\left\langle\psi \nabla \chi_{i}\right\rangle=\kappa|\mu|\left[\frac{\left(p_{j} \Psi_{j}\right)}{\lambda_{j}}-\frac{\left(p_{i} \psi_{i}\right)}{\lambda_{i}}\right]+\frac{\eta|\mu|}{2}\left[\frac{\int\left(p_{i} \psi_{i}\right) d \mu^{\prime}}{\lambda_{i}}-\frac{\int\left(p_{j} \psi_{j}\right) d \mu^{\prime}}{\lambda_{j}}\right]
$$

with two proposed formulas for the weighting parameters $\kappa$ and $\eta$. The spherical equations then are

$$
\left[\frac{1}{c} \frac{\partial}{\partial t}+\mu \frac{\partial}{\partial \rho}+\frac{\left(1-\mu^{2}\right)}{\rho} \frac{\partial}{\partial \mu}+\sigma_{a i}\right]\left(p_{i} \psi_{i}\right)=p_{i} S_{i}+\frac{c \sigma_{a i}}{2}\left(p_{i} \phi_{i}\right)+\left\langle\psi \nabla \chi_{i}\right\rangle
$$

and 


$$
\frac{\partial}{\partial t}\left(p_{i} \phi_{i}\right)=-c \sigma_{a i}\left(p_{i} \phi_{i}\right)+\sigma_{a i} \int_{-1}^{1}\left(p_{i} \Psi_{i}\right) d \mu
$$

with $i, j=1,2$ and $i \neq j$, with the coupling term $\left\langle\psi \nabla \chi_{i}\right\rangle$ given by the closure relation Eq. (101) and the ensemble averages given by Eqs. (99) and (100). Once again the two variations on the forms for the weighting parameters $\kappa$ and $\eta$ are given by Eqs. (73) and (74) (variation 1) and by Eqs. (77) and (78) (variation 2).

\subsection{Numerical Results}

Now investigate how the various model formulations in Sec. (11.0) perform relative to the benchmark.

\subsection{Numerical Techniques}

All methods are solved using a 1-D spherical generalization of diamond difference for discretization of the gradient operator - see Appendix B or reference [17], and implicit time-discretization for the temperature and specific intensity time derivatives. The angular variable $\mu$ is treated by standard discrete ordinate methods - again see Appendix B or reference [17]. At every time step, the temperature and specific intensity were updated to the same time stamp via an inner (fixed point) iteration, of fixed length. The maximum size $\Delta \rho$ of the radial grid spacing was chosen according to the same relation as was used for the slab geometry case. Here it does not necessarily guarantee positivity, but lacking an equivalent formula for the spherical geometry it was used anyway. The time step $\Delta t$ is again fixed at the value $\Delta t=1.0 e-12$ seconds for the same reasons explained in the 
slab geometry section.

In all of the numerical tests for the 1-D sphere, the temperature of the core was held to be constant. Also were specified a constant and isotropic radiation source in the core only. However, the radiation specific intensity within the core is allowed to change. This retains the effect of the cross-problem connectivity, as discussed in Sec. (10.0), inherent to this geometry. Because this introduces an effect not present in the 1-D geometry runs, it makes it difficult to compare the 1-D slab with the 1-D spherical runs. Because of time constraints no tests were done with the radiation field in the core held constant - what would essentially be a curved slab geometry...not a true sphere.

\subsection{Results - Constant Opacities without Temperature Coupling}

The Standard Model has never before been used in the 1-D spherical geometry. If this were a 1-D slab geometry with HM statistics and constant opacities, then the Standard Model would in principle give the exact result. But this is not a 1-D slab geometry and hence the statistics are not homogeneous as explained in Sec. (10.0). So in order to explore the performance of the Standard Model in this new geometry without the additional complication of coupling to material temperature, first compute solutions to the different methods without material temperature coupling. Table (3) has the physical parameters used in these runs.

The radius of the core is given by $R_{\text {Core }}$ and the thickness of the surrounding mixing region by $L$. The radiation source is constant, isotropic, specified by $S_{\text {Core }}$, and is zero everywhere outside the core. 
Table 3: Parameters for 1-D Sphere without Temperature

\begin{tabular}{|c|c|c|}
\hline \multicolumn{3}{|c|}{$\sigma_{a 1}=1000 / \mathrm{cm} \quad \sigma_{a 2}=5 / \mathrm{cm}$} \\
& $\sigma_{a \text { Core }}=\sigma_{a 2}$ \\
& $S_{\text {Core }}=0.3 \mathrm{KeV}$ \\
& $R_{\text {Core }}=\mathbf{0 . 1} \mathrm{cm}$ \\
& $L=0.15 \mathrm{~cm}$ \\
\hline Figure \# & $\lambda_{1}(\mathbf{c m})$ & $\lambda_{2}(\mathbf{c m})$ \\
\hline \hline 32,36 & $5.0 \mathrm{e}-3$ & $5.0 \mathrm{e}-1$ \\
\hline 33,37 & $5.0 \mathrm{e}-4$ & $5.0 \mathrm{e}-2$ \\
\hline 34,38 & $5.0 \mathrm{e}-5$ & $5.0 \mathrm{e}-3$ \\
\hline 35,39 & $5.0 \mathrm{e}-6$ & $5.0 \mathrm{e}-4$ \\
\hline
\end{tabular}

Just as in the slab geometry, the volume ratio for material $i$ at any given point is equal to the probability of finding that material there, i.e. $V_{i}=P_{i}=\frac{\lambda_{i}}{\left(\lambda_{1}+\lambda_{2}\right)}$. And again the different $\lambda_{i}$ were specifically chosen so that the volume ratios were the same for all the test runs, just as in the slab geometry runs. Hence the results for the atomic mix model will again be the same for all test runs.

Each of the figures (32) through (35) has three curves. Curve (A) is the benchmark value. Curve (B) is the value returned by the atomic mix model Eq. (95). Curve (C) is the result from the standard model adaptation given by Eq. (97). Because there is no material temperature or scattering for these runs, there is no adaptation of the Su-Pomraning model.

Because there is no material temperature, only the radiation transport equation is being 
solved. The results, Figs. (32) through (35), are plotted as plots of $\frac{\langle\text { Trans }\rangle}{\operatorname{caT}_{o}^{4}}$ versus time step as computed for the three different models and the benchmark, where

$$
\text { Trans }=\int_{0}^{1} \Psi_{\text {Surface }} \mu d \mu
$$

In other words, it shows the time evolution of the ensemble average of the radiant energy streaming off the surface of the sphere as shown in Fig. (30). It is also scaled to $T_{o}=0.03 \mathrm{KeV}$. This is the same value used for the initial material temperature in the temperature coupled runs made later in this chapter. The scaling was kept the same just for consistency.

The values of $\frac{\langle\operatorname{Trans}\rangle}{c a T_{o}^{4}}$ were plotted with time on a log scale for these runs. This was only done for these runs to better bring out the differences between the benchmark and the Standard Model.

The benchmark variance in the transmission is shown in Figs. (36) through (39) and is a plot of $\frac{\sqrt{\left\langle(\operatorname{Trans})^{2}\right\rangle-\langle\text { Trans }\rangle^{2}}}{\langle\text { Trans }\rangle}$ as a function of time step. The time step axis is on a $\log$ scale. 
FIGURE 32. 〈Trans $\rangle$ versus Time Step without Temperature Coupling

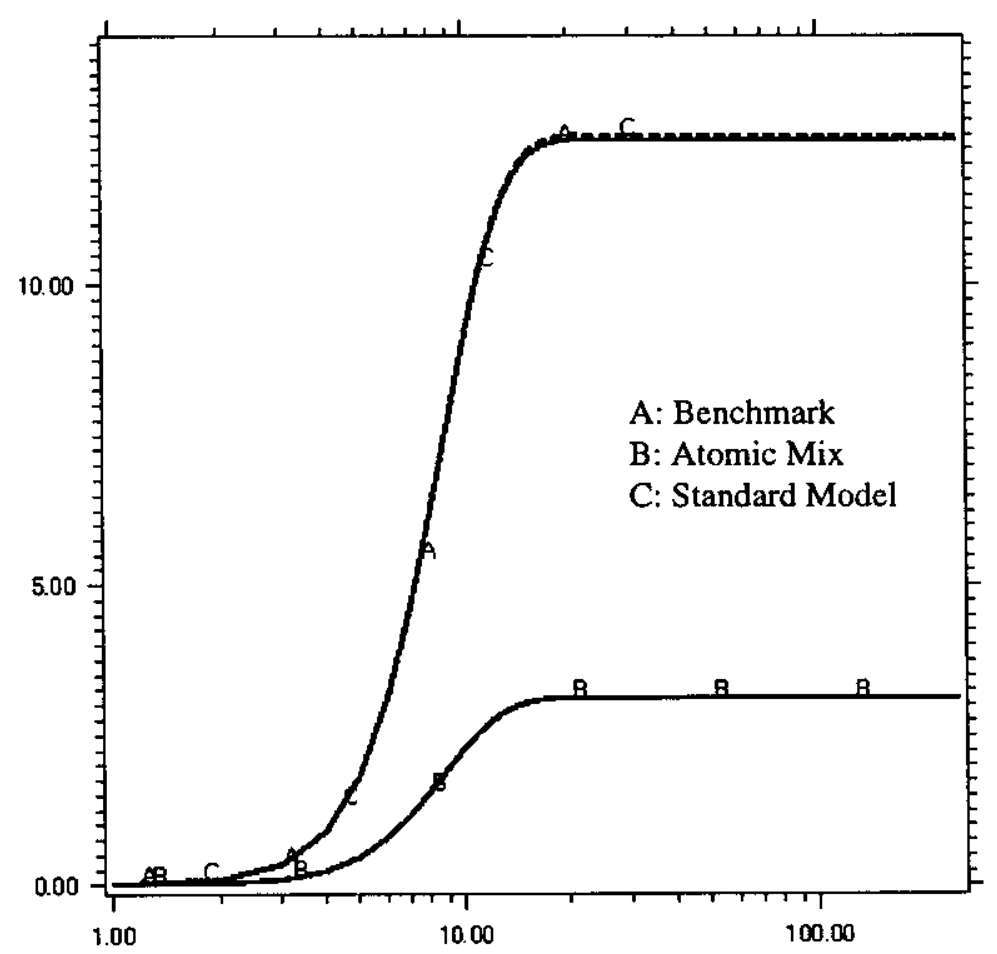

FIGURE 33. $\langle$ Trans $\rangle$ versus Time Step without Temperature Coupling

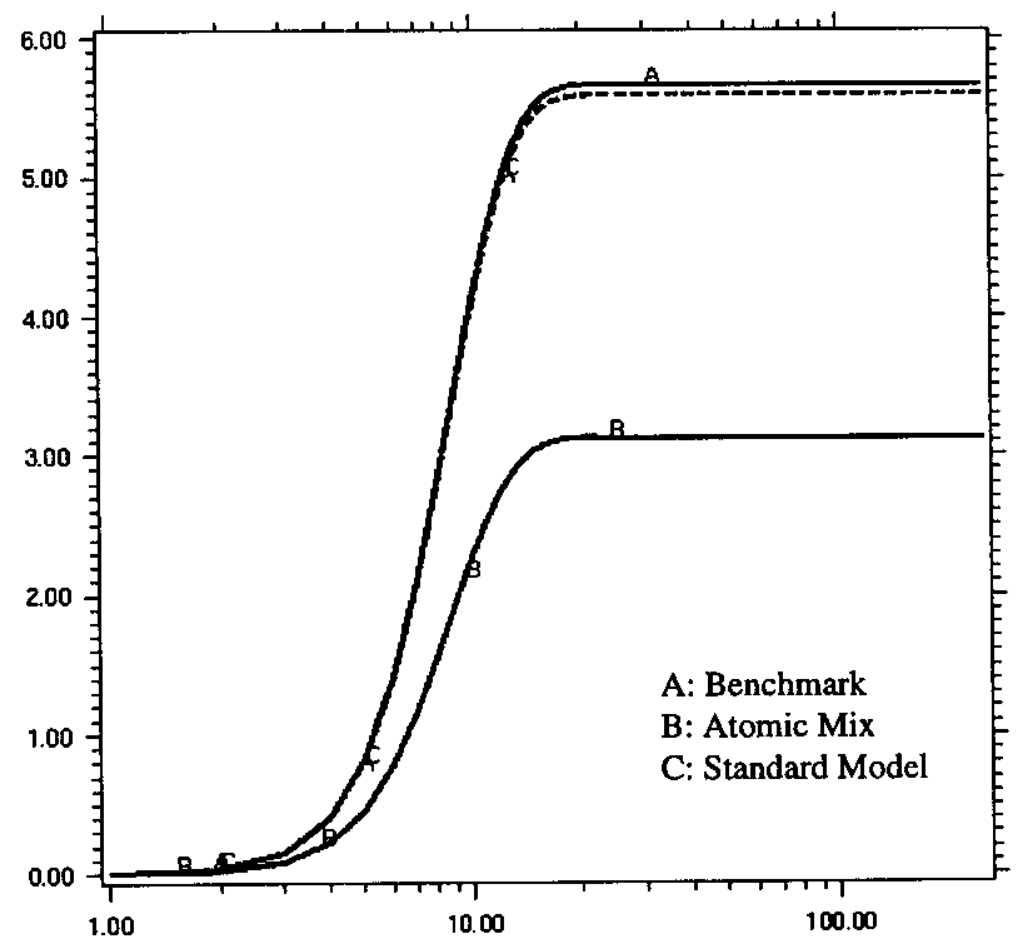


FIGURE 34. 〈Trans〉 versus Time Step without Temperature Coupling

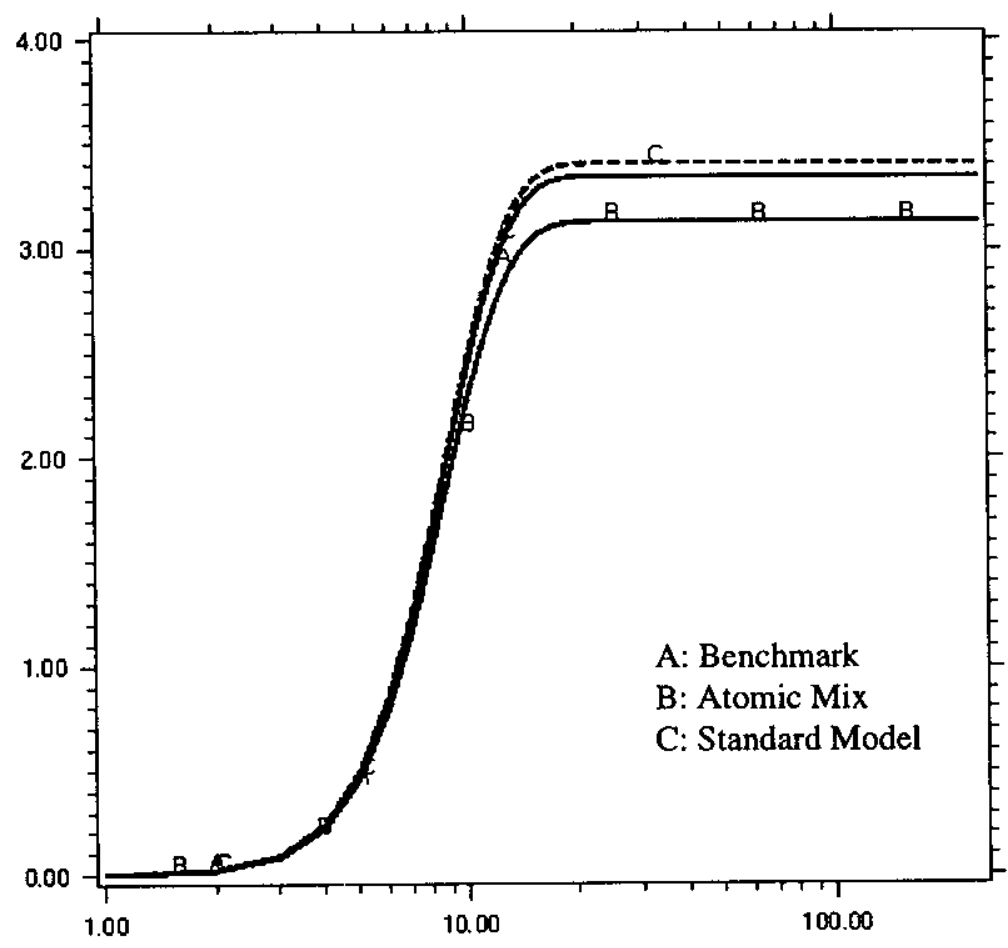

FIGURE 35. $\langle$ Trans $\rangle$ versus Time Step without Temperature Coupling

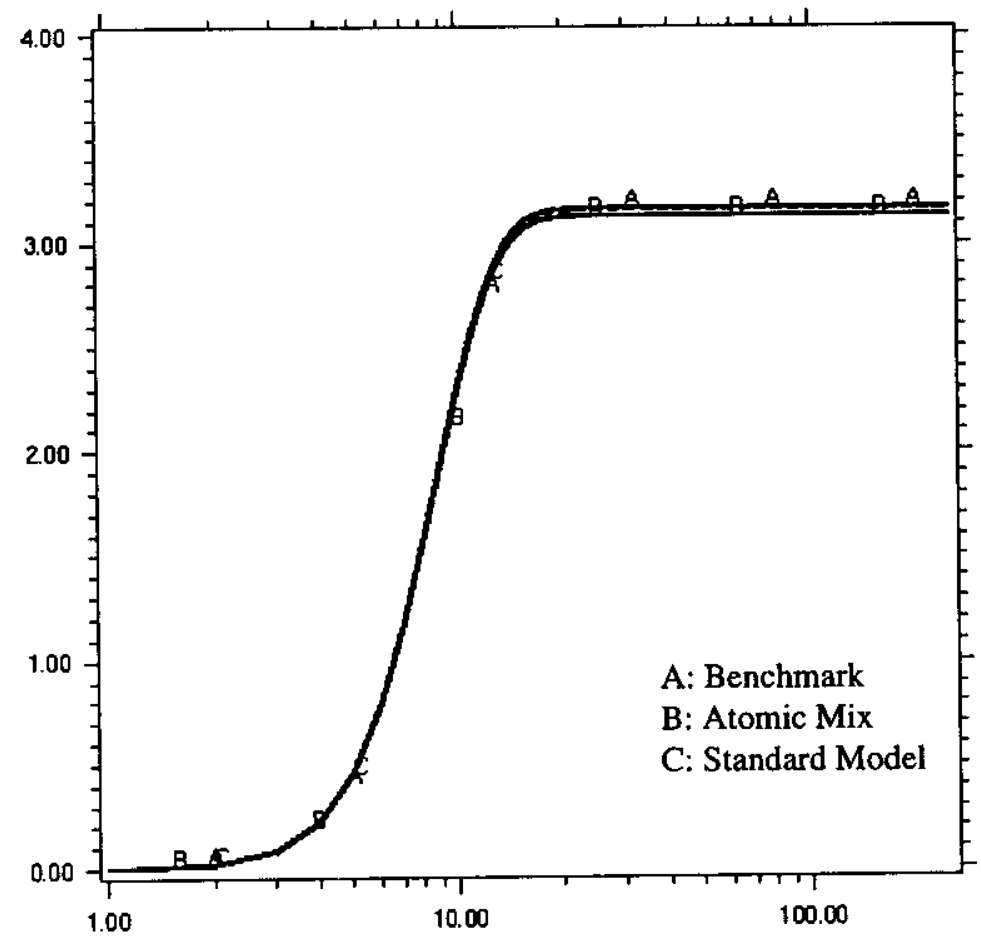


FIGURE 36. Benchmark Variance without Temperature Coupling

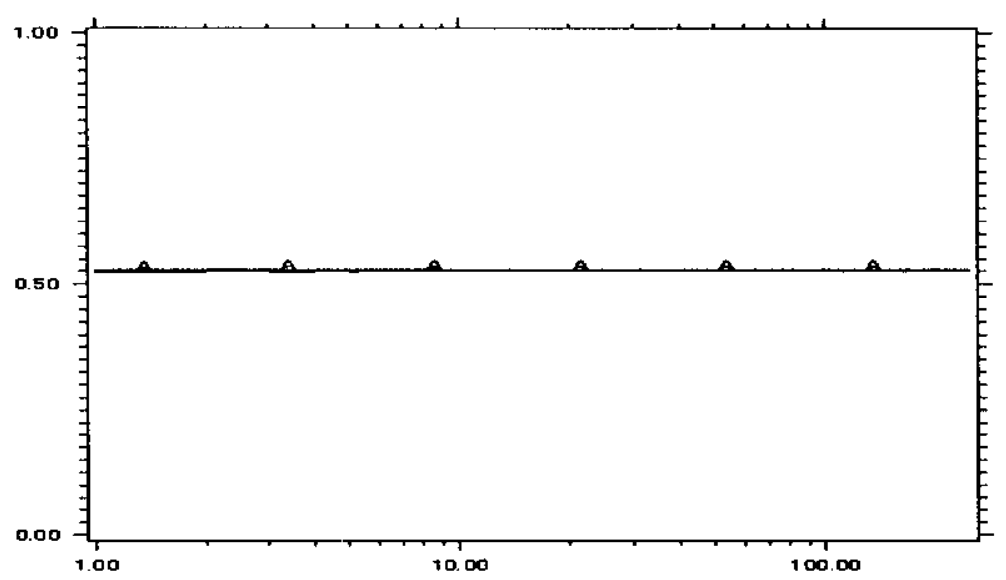

FIGURE 37. Benchmark Variance without Temperature Coupling

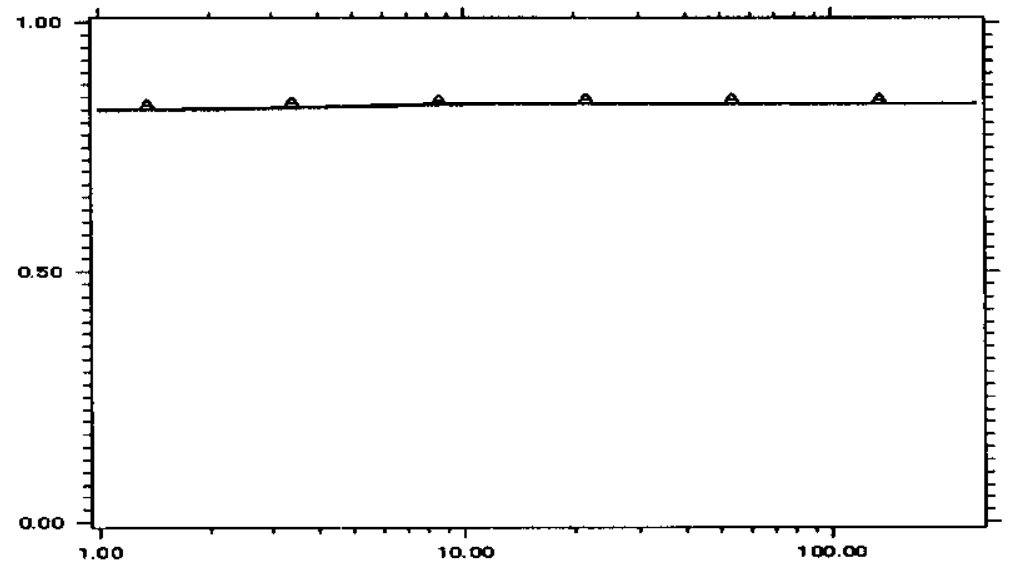

FIGURE 38. Benchmark Variance without Temperature Coupling

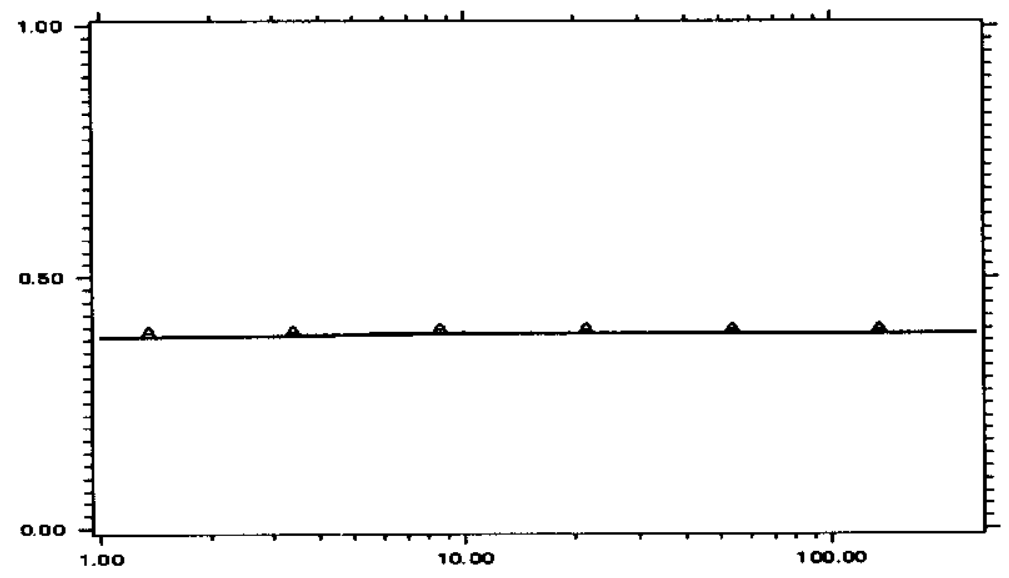


FIGURE 39. Benchmark Variance without Temperature Coupling

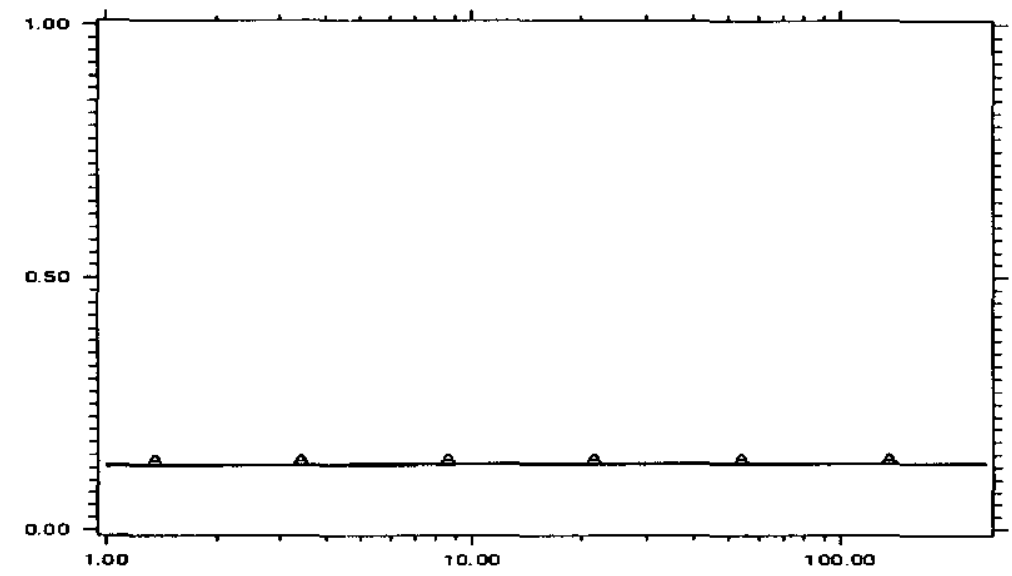

The Standard Model performed extremely well here. It fact it performed remarkably well. Evidently the variation from HM statistics that is introduced by going to the 1-D spherical geometry is not nearly as important as was the introduction of temperature dependent opacities in the slab geometry.

Notice that all the different methods converge to the atomic mix answer in the small correlation length limit, i.e as the $\lambda_{i}$ go to zero. Notice too that the atomic mix answer is now getting the transient correct in this limit. This is because the temperature equation is not part of the picture here and the purely local nature of the temperature equation is not introducing error into the atomic mix answer. Also notice that because the atomic mix approximation is correct here in the small correlation limit, the variances are all going to zero in this limit as well. Hence the big hump in the transient for the variance is gone.

For these runs without the material heating there is no scattering type of effect. There is no redistribution in direction. So a photon starts out going one direction and keeps going in that direction unless absorbed. This means that the cross problem connectivity discussed above is not present in these runs. All the radiation source is in the core so photons 
never stream back into the core from the mixing region. They only stream outward. This problem is more of a curved slab than a sphere. This might help explain why the standard model performs so well here.

Notice that the relative position of the atomic mix model is the same here as it was in the 1-D slab geometry runs. It continues to over absorb radiation resulting in a lower steady state outgoing flux than the benchmark or the standard model.

The next thing to do is turn the coupling to material temperature back on, still keeping the opacities constant, and redo the runs.

\subsection{Results - Constant Opacities with Temperature Coupling}

Here are presented the results of the different methods in a 1-D spherical geometry with the material temperature coupling turned back on but with the opacities still held constant. The parameters of the problem are given in Table (4). Again the volume ratios of the two

Table 4: Parameters for 1-D Sphere with Temperature - Constant Opacities

\begin{tabular}{|c|c|c|}
\hline \multicolumn{2}{|c|}{$\sigma_{a 1}=1000 / \mathrm{cm} \quad \sigma_{a 2}=5 / \mathrm{cm}$} \\
\multicolumn{3}{|c|}{$\sigma_{a \text { Core }}=\sigma_{a 2}$} \\
& $S_{\text {Core }}=0.3 \mathrm{KeV}$ \\
& $T_{\text {Core }}=0.3 \mathrm{KeV}$ \\
& $T_{o}=0.03 \mathrm{Kev}$ \\
& $R_{\text {Core }}=\mathbf{0 . 1} \mathrm{cm}$ \\
& & \\
\multicolumn{3}{|c|}{$=\mathbf{0 . 1 5} \mathrm{cm}$} \\
\hline Figure \# & $\lambda_{1}(\mathbf{c m})$ & $\lambda_{2}(\mathrm{~cm})$ \\
\hline \hline $40,44,48$ & $5.0 \mathrm{e}-3$ & $5.0 \mathrm{e}-1$ \\
\hline $41,45,49$ & $5.0 \mathrm{e}-4$ & $5.0 \mathrm{e}-2$ \\
\hline $42,46,50$ & $5.0 \mathrm{e}-5$ & $5.0 \mathrm{e}-3$ \\
\hline
\end{tabular}


Table 4: Parameters for 1-D Sphere with Temperature - Constant Opacities

\begin{tabular}{|c|c|c|}
\hline \multicolumn{3}{|c|}{$\begin{array}{c}\sigma_{a 1}=1000 / \mathrm{cm} \quad \sigma_{a 2}=5 / \mathrm{cm} \\
\sigma_{a C o r e}=\sigma_{a 2} \\
S_{\text {Core }}=0.3 \mathrm{KeV} \\
T_{\text {Core }}=0.3 \mathrm{KeV} \\
T_{o}=0.03 \mathrm{Kev} \\
R_{\text {Core }}=0.1 \mathrm{~cm} \\
L=0.15 \mathrm{~cm}\end{array}$} \\
\hline Figure \# & $\lambda_{1}(\mathrm{~cm})$ & $\lambda_{2}(\mathrm{~cm})$ \\
\hline $43,47,51$ & $5.0 \mathrm{e}-6$ & $5.0 e-4$ \\
\hline
\end{tabular}

material were held constant as the size of the material chunks were made smaller. The material temperature, isotropic radiation source and material properties in the core were constant and given by the values in Table (4). However the radiation specific intensity in the core was allowed to change as photons were redirected via thermal emission and streamed back into the core from the mixing region. This represents a 1-D sphere now not just a curved slab geometry as discussed above.

Each of the figures (40) through (47) have four curves. Curve (A) is the benchmark value. Curve (B) is the value returned by the atomic mix model, Eqs. (95) and (96). Curve (C) is the result from the standard model adaptation given by Eqs. (97) and (98). Curve (D) is the result from the adaptation of the Su-Pomraning model, Eqs. (101) through (103), with the effective scattering Eq. (69) used in calculation of the weighting factors via Eqs. (73) and (74), i.e. variation number 1. Because the method with weighting factors Eqs. (77) and (78), i.e. variation number 2, was not stable under source iteration (refer to Sec. (9.3)) it was not used. 
Figures (40) through (43) are plots of $\frac{\langle\varphi\rangle}{a T_{o}^{4}} \quad$ versus time step as computed for the four different methods. In other words, the time evolution of the ensemble average of the material temperature energy density scaled to the initial material temperature $T_{o}$, as computed at the surface of the sphere, as shown in Fig. (30).

Figures (44) through (47) are plots of $\frac{\langle\operatorname{Trans}\rangle}{c a T_{o}^{4}}$ versus time step as computed for the three different models and the benchmark, where

$$
\operatorname{Trans}=\int_{0}^{1}(\psi(t, z=L, \mu)) \mu d \mu
$$

In other words, the time evolution of the ensemble average of the radiant energy streaming off the surface of the sphere and scaled to the initial material temperature $T_{o}$.

Figures (48) through (51) are plots of the variance in the benchmark for material temperature energy density and radiation specific intensity as scaled by the answer for each time step. In other words it is plots of percentage variance given by $\frac{\sqrt{\left\langle\varphi^{2}\right\rangle-\langle\varphi\rangle^{2}}}{\langle\varphi\rangle}$ and $\frac{\sqrt{\left\langle(\text { Trans })^{2}\right\rangle-\langle\text { Trans }\rangle^{2}}}{\langle\text { Trans }\rangle}$ as a function of time step. The time step axis for the variances is on a $\log$ scale. 
FIGURE 40. $\langle\varphi\rangle$ versus Time Step - Constant Opacities

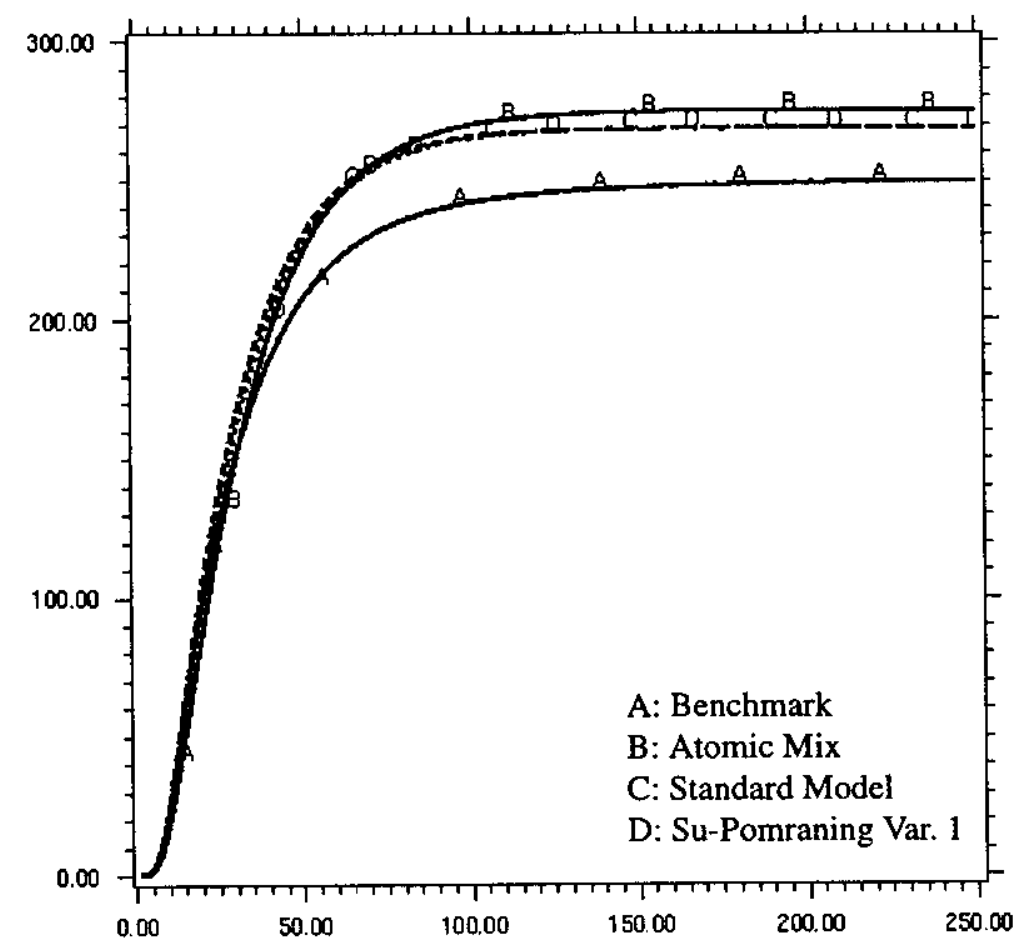

FIGURE 41. $\langle\varphi\rangle$ versus Time Step - Constant Opacities

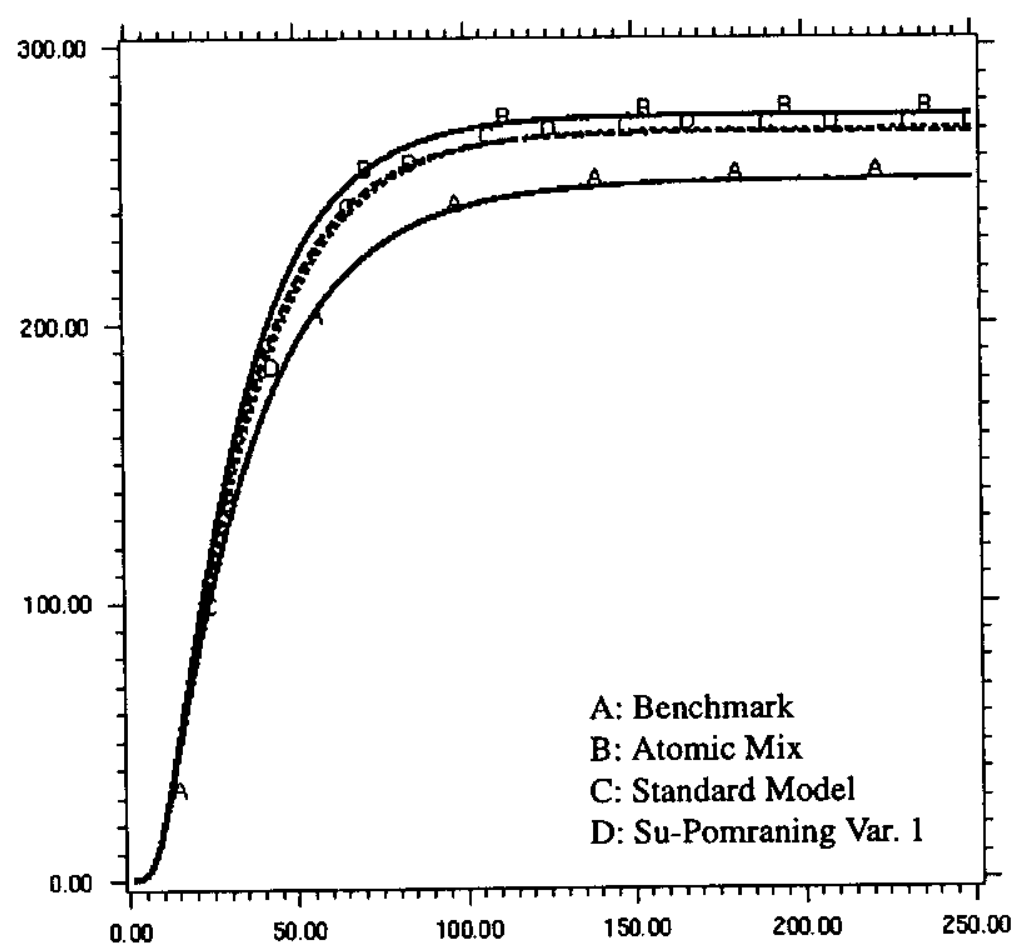


FIGURE 42. $\langle\varphi\rangle$ versus Time Step - Constant Opacities

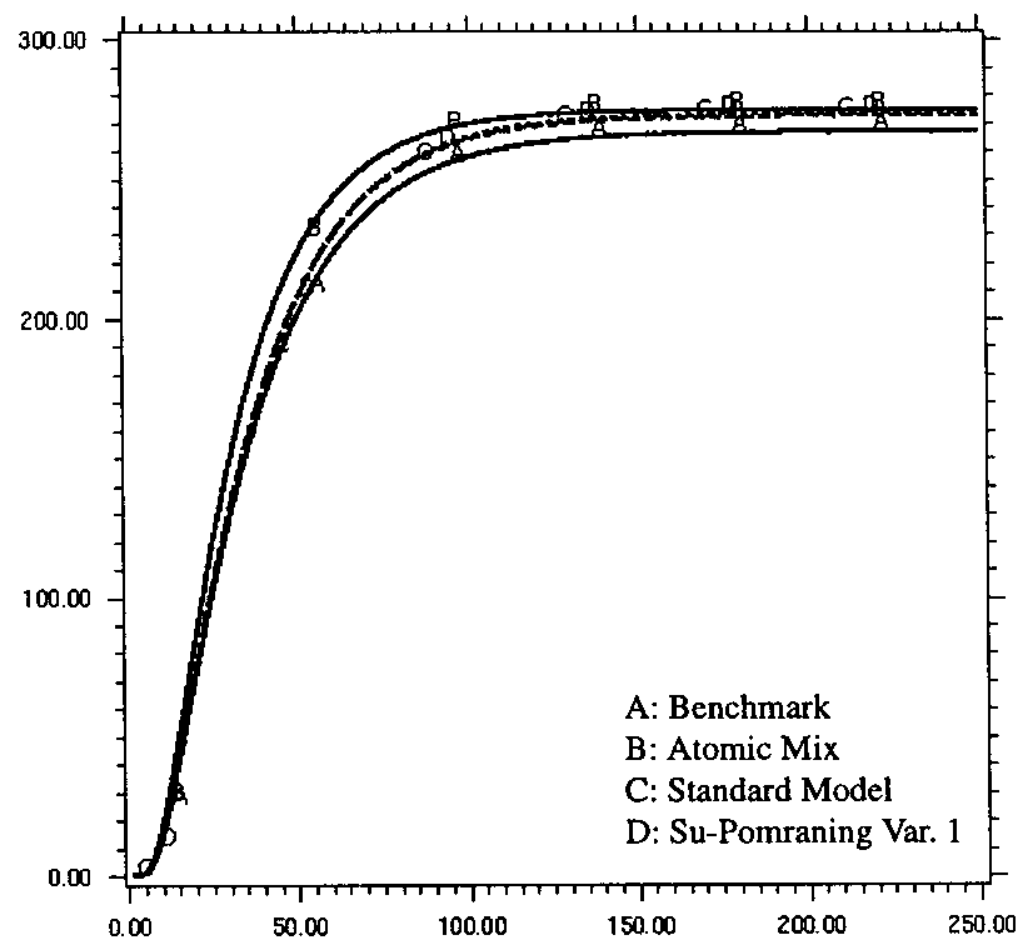

FIGURE 43. $\langle\varphi\rangle$ versus Time Step - Constant Opacities

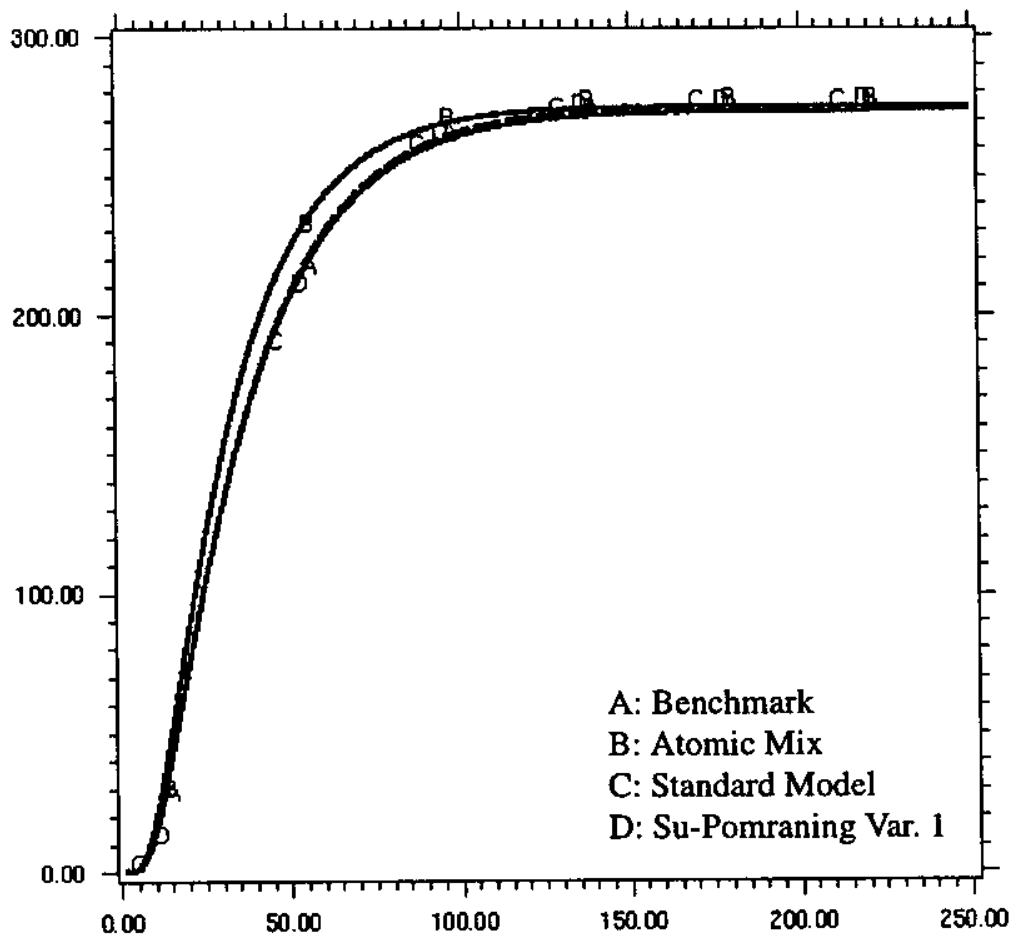


FIGURE 44. 〈Trans $\rangle$ versus Time Step - Constant Opacities

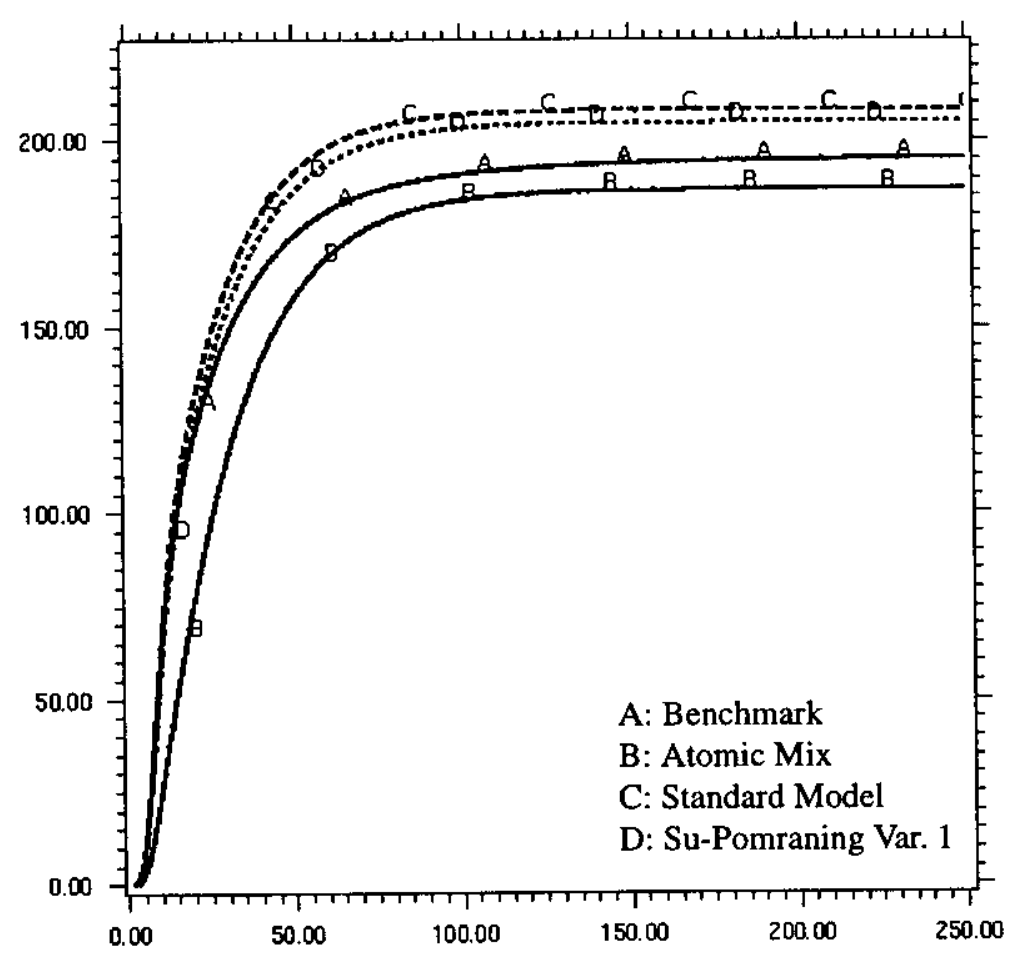

FIGURE 45. 〈Trans $\rangle$ versus Time Step - Constant Opacities

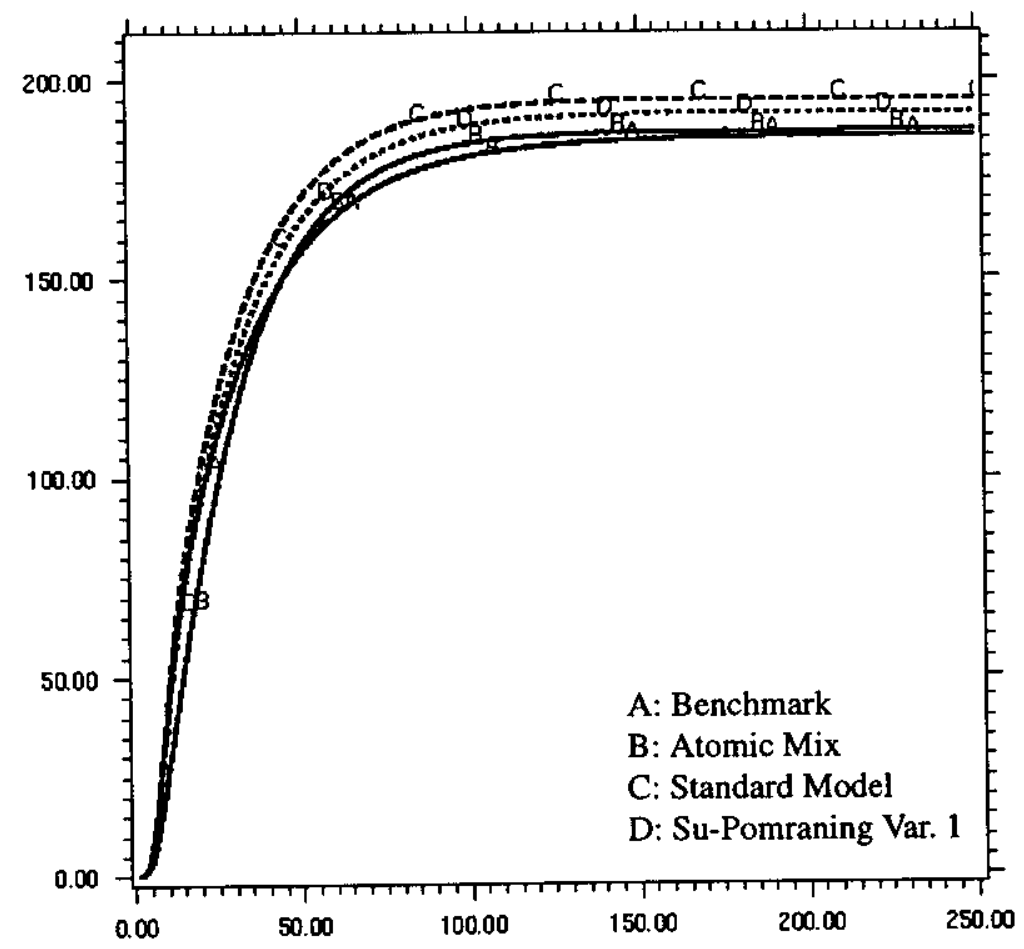


FIGURE 46. 〈Trans $\rangle$ versus Time Step - Constant Opacities

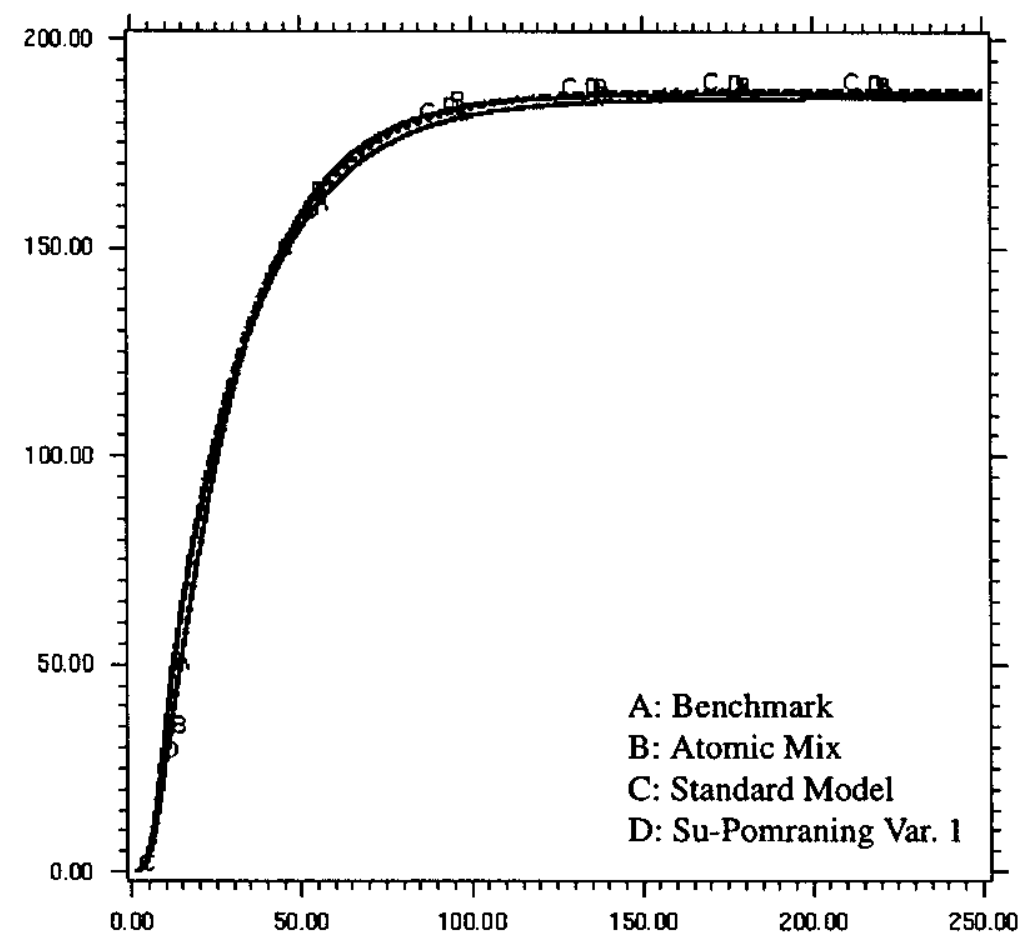

FIGURE 47. 〈Trans $\rangle$ versus Time Step - Constant Opacities

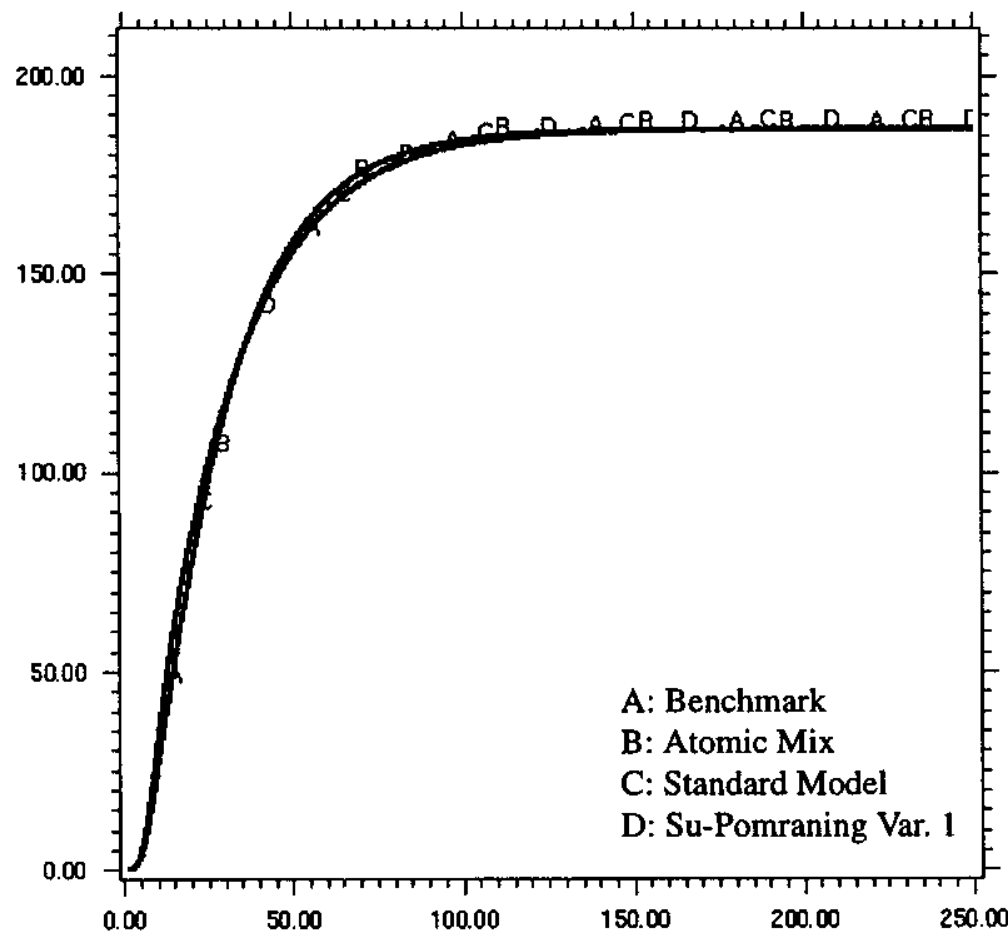


FIGURE 48. Benchmark Variances - Constant Opacities

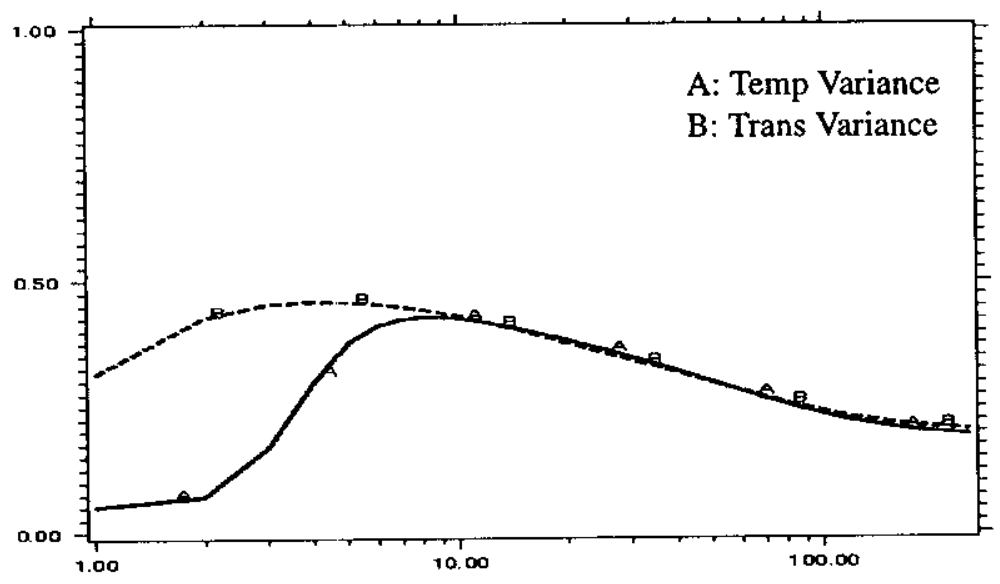

FIGURE 49. Benchmark Variances - Constant Opacities

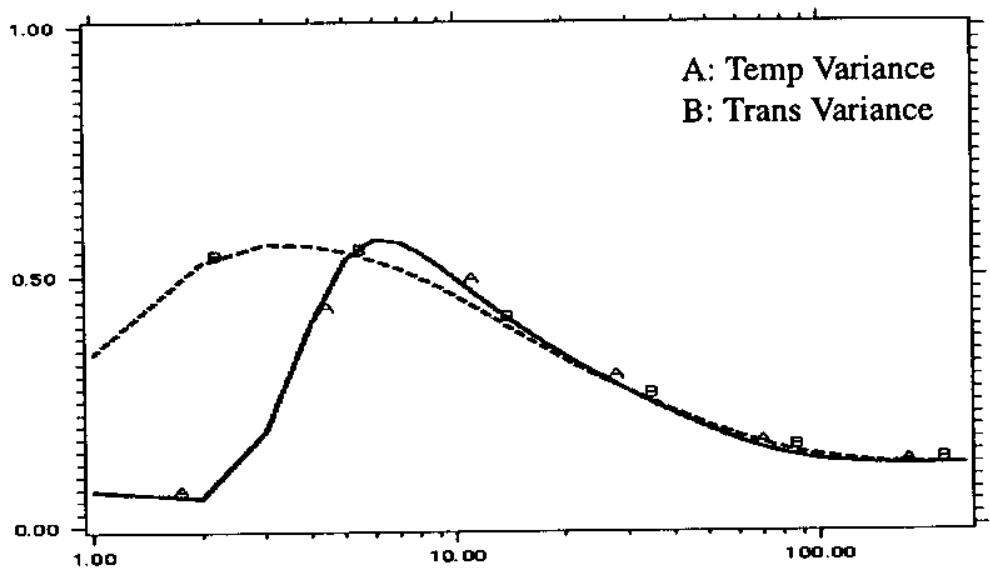

FIGURE 50. Benchmark Variances - Constant Opacities

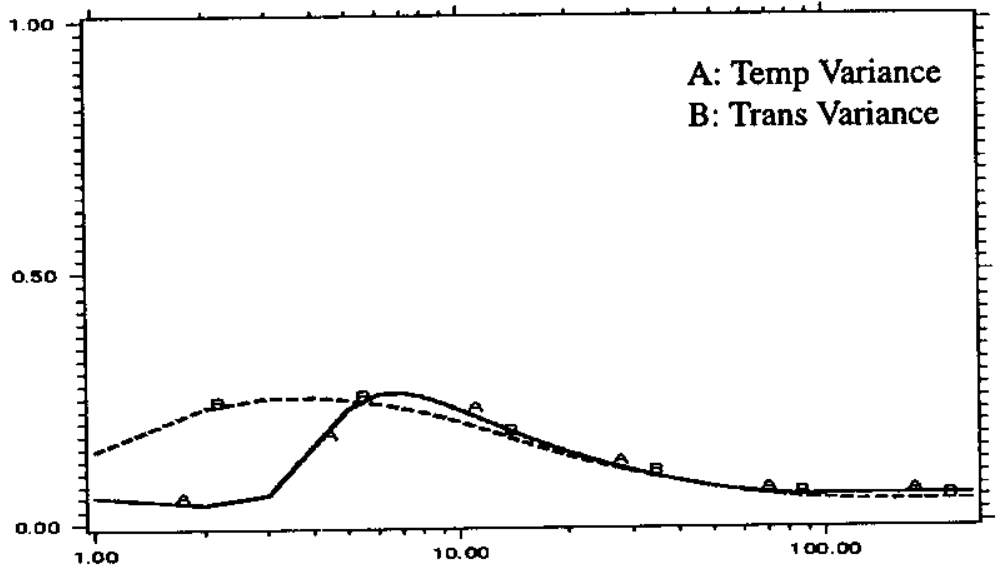


FIGURE 51. Benchmark Variances - Constant Opacities

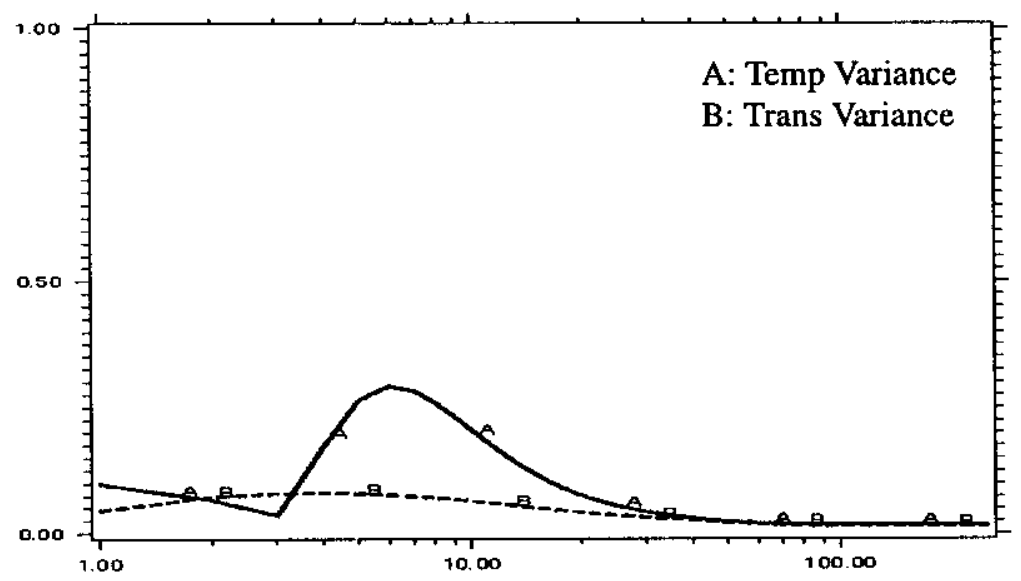

First notice that as the size of the material chunks (shell widths) is made smaller, i.e. the small correlation length limit, all of the methods converge to the same steady state answer. This is a good check and is what should be happening. Also notice that the atomic mix model (B) once again does not get the transient stage correct, even though the steady state answers are converging. This is precisely for the same reasons explained in Sec. (9.3).

Also notice that because the atomic mix is once again not the correct small correlation limit, the hump in the transient period for the variance is back. But it still goes to zero in the steady state in this limit as it should.

With regards to the temperature, the standard model (C) and Su-Pomraning with effective scattering (D) have almost identical performance and only slightly better than the atomic mix model (B). However for the radiation, the atomic mix actually does a better job. Notice too that the relative performance of the atomic mix model has changed. It now is resulting in steady state temperatures that are consistently higher than the other models. This was not the case in the 1-D slab geometry. Why this is happening is not yet clear, but 
it may well be connected to the fact that the sphere now has the connectivity across the source (the core) not contained in the 1-D slab.

The standard model and the Su-Pomraning with effective scattering show little if any improvement over the atomic mix model for this problem.

\subsection{Results - Temperature Dependent opacities}

Now redo the runs from the previous section but allow the opacities for the two materials to change with temperature. Again use the same temperature dependence as was used in the 1-D slab geometry runs. Allow the opacities of the two materials to depend on temperature as

$$
\sigma_{a i}=\frac{C_{i}}{T^{3.5}},
$$

with $C_{1}=1.07 \times 10^{28}$ and $C_{2}=1.73 \times 10^{22}$. The remaining parameters are shown in Table (5). Again the source of radiation in the core is constant and isotropic, the temperature in the core is held fixed (and hence so are the opacities) and the radiation is allowed to stream across the core region. And again the volume ratios of the two materials are held constant while the average shell widths of the two materials are made smaller.

Each of Figs. (52) through (59) has four curves. Curve (A) is the benchmark value. Curve (B) is the value returned by the atomic mix model, Eqs. (95) and (96). Curve (C) is the result from the standard model adaptation given by Eqs. (97) and (98). Curve (D) is the result from the adaptation of the Su-Pomraning model, Eqs. (101) through (103), with the effective scattering Eq. (69) used in calculation of the weighting factors via Eqs. (73) and 
(74), i.e. variation number 1.

Table 5: Parameters for 1-D Sphere with Temperature - Variable Opacities

\begin{tabular}{|l|l|l|}
\hline \multicolumn{3}{|c|}{$\sigma_{\text {aCore }}=\sigma_{a 2}$} \\
\multicolumn{3}{|c|}{$S_{\text {Core }}=0.3 \mathrm{KeV}$} \\
\multicolumn{3}{|c|}{$T_{\text {Core }}=0.3 \mathrm{KeV}$} \\
\multicolumn{3}{|c|}{$T_{o}=0.03 \mathrm{Kev}$} \\
\multicolumn{3}{|c|}{$R_{\text {Core }}=0.1 \mathrm{~cm}$} \\
\multicolumn{3}{|c|}{ 0.15 cm } \\
\hline Figure \# & $\lambda_{1}(\mathbf{c m})$ & $\lambda_{2}(\mathbf{c m})$ \\
\hline \hline $52,56,60$ & $5.0 \mathrm{e}-3$ & $5.0 \mathrm{e}-1$ \\
\hline $53,57,61$ & $5.0 \mathrm{e}-4$ & $5.0 \mathrm{e}-2$ \\
\hline $54,58,62$ & $5.0 \mathrm{e}-5$ & $5.0 \mathrm{e}-3$ \\
\hline $55,59,63$ & $5.0 \mathrm{e}-6$ & $5.0 \mathrm{e}-4$ \\
\hline
\end{tabular}

Again the method with weighting factors Eqs. (77) and (78), i.e. variation number 2, was not used.

Again Trans is defined by Eq. (105) and all values are scaled to $T_{o}$ just as in all previous runs. Figures (53) through (56) are plots of $\frac{\langle\varphi\rangle}{a T_{o}^{4}} \quad$ versus time step as computed for the four different methods at the surface of the sphere. Figures (57) through (59) are plots of $\frac{\langle\operatorname{Trans}\rangle}{c a T_{o}^{4}}$ versus time step as computed for the four different methods at the surface the sphere. The benchmark variances for $\langle\varphi\rangle$ and $\langle\psi\rangle$ are plotted versus time step Figs. (60) through (63). Again these are plotted with the time axis on a log scale. 
FIGURE 52. $\langle\varphi\rangle$ versus Time Step - Variable Opacities

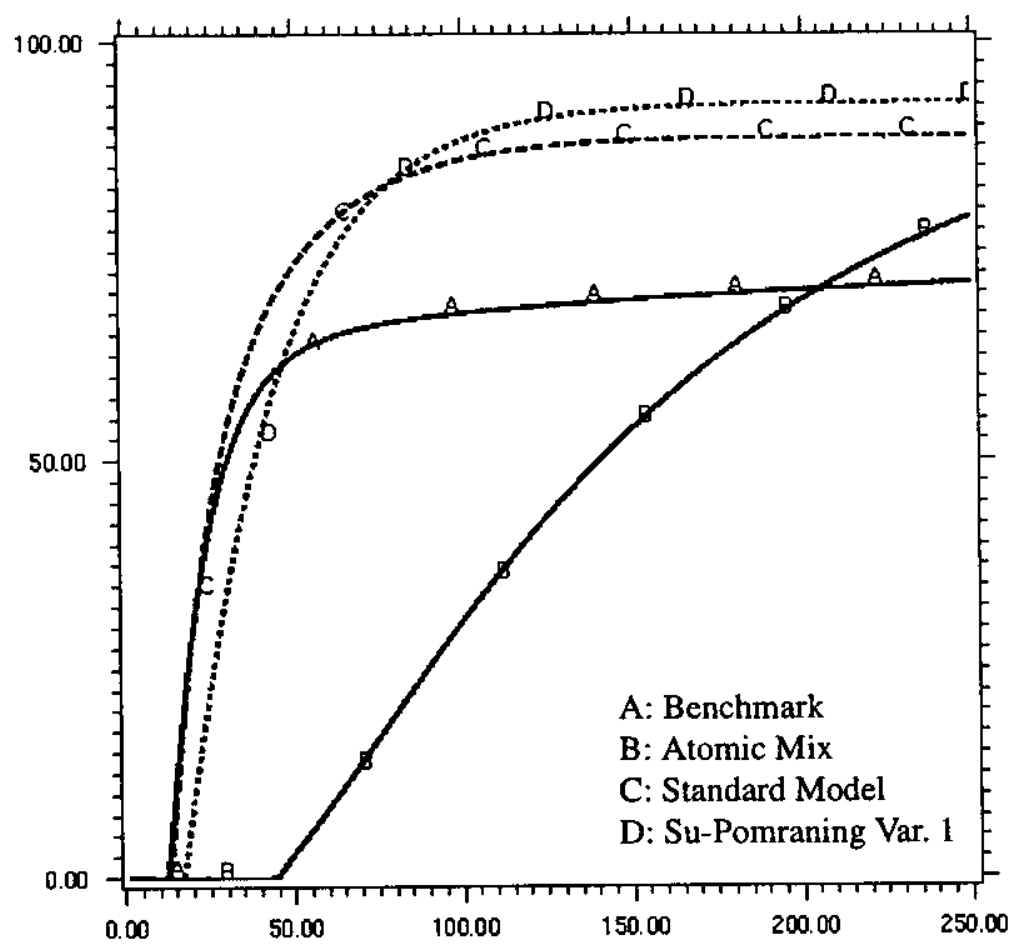

FIGURE 53. $\langle\varphi\rangle$ versus Time Step - Variable Opacities

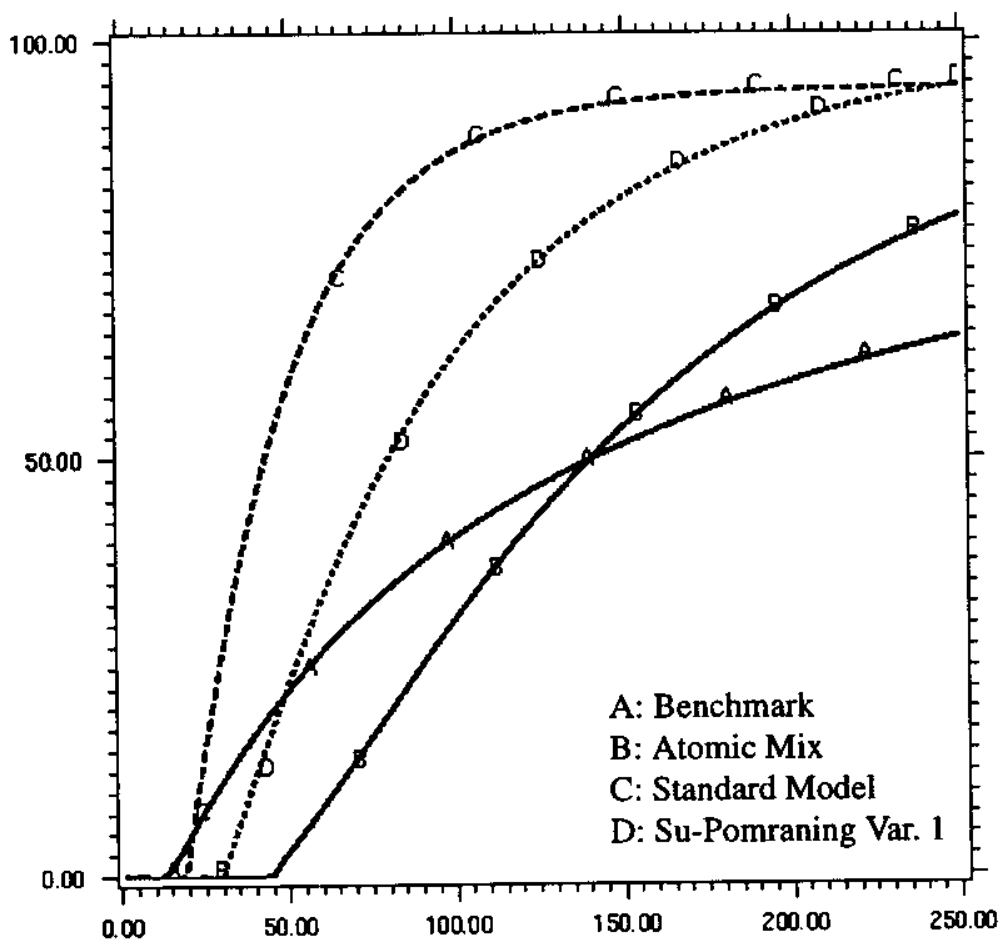


FIGURE 54. $\langle\varphi\rangle$ versus Time Step - Variable Opacities

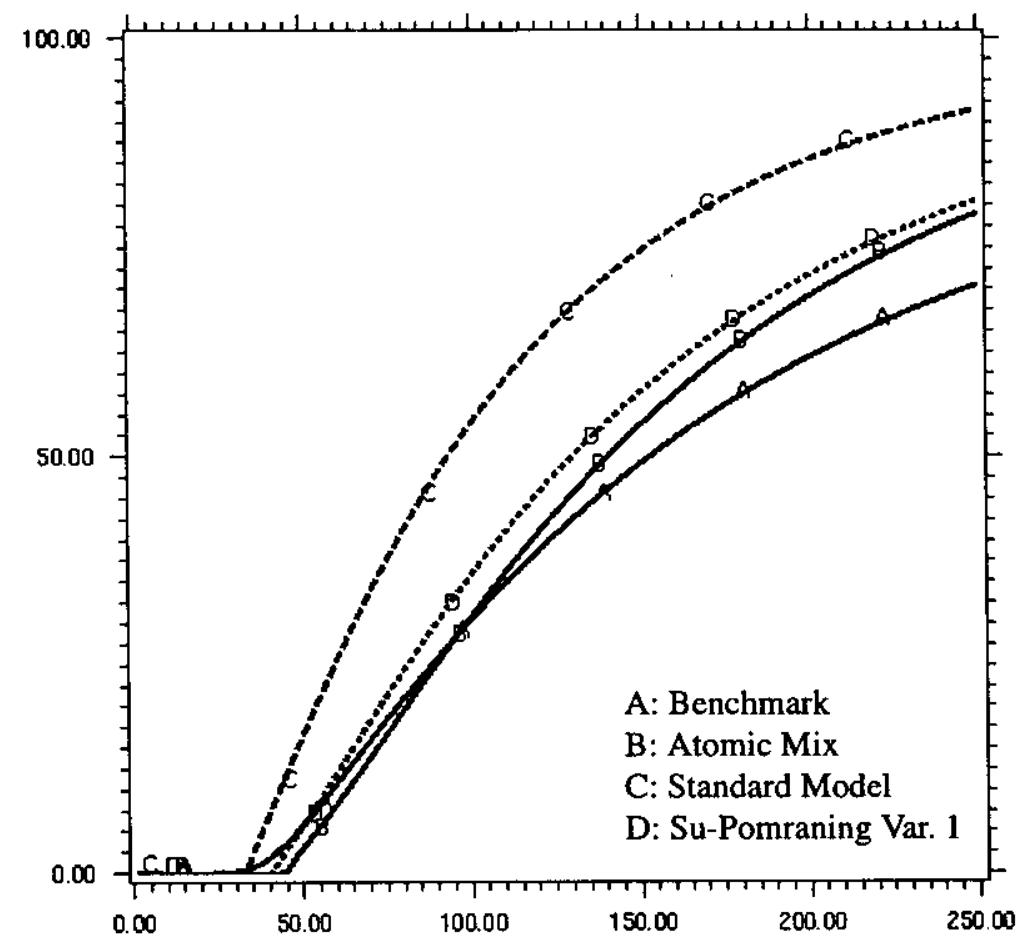

FIGURE 55. $\langle\varphi\rangle$ versus Time Step - Variable Opacities

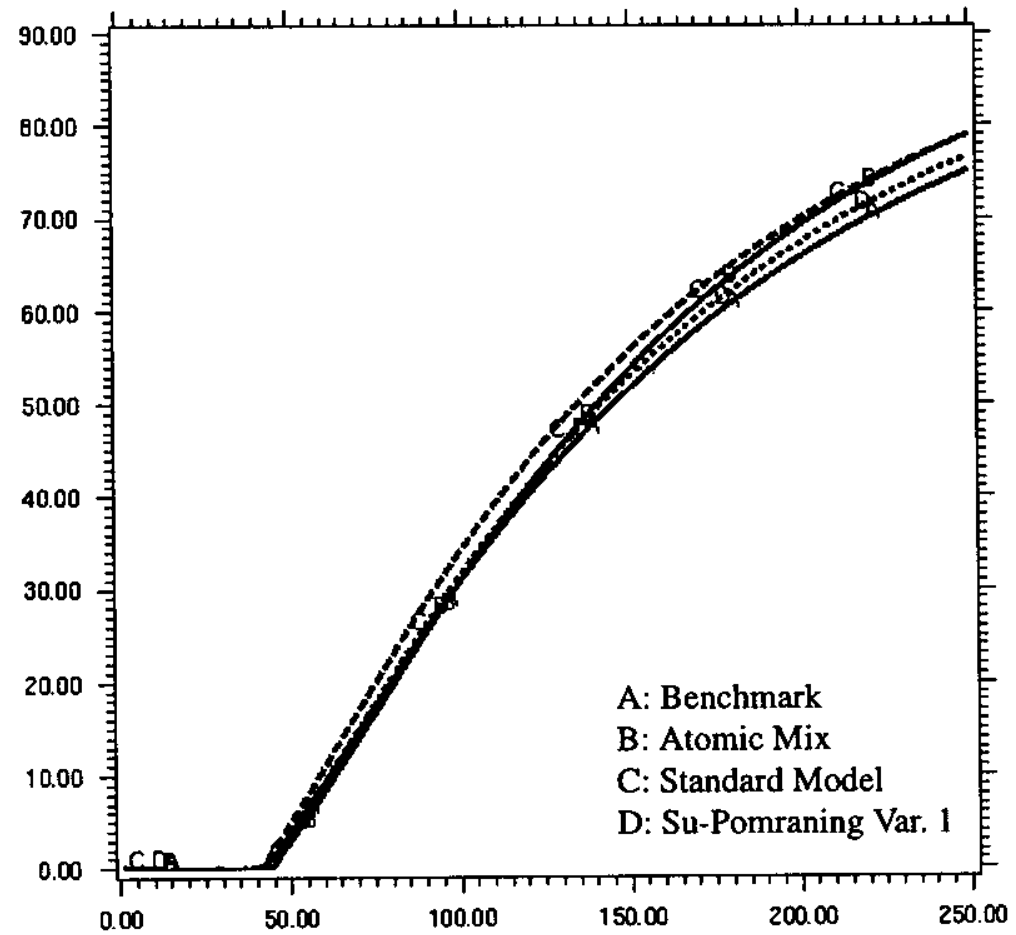


FIGURE 56. 〈Trans〉 versus Time Step - Variable Opacities

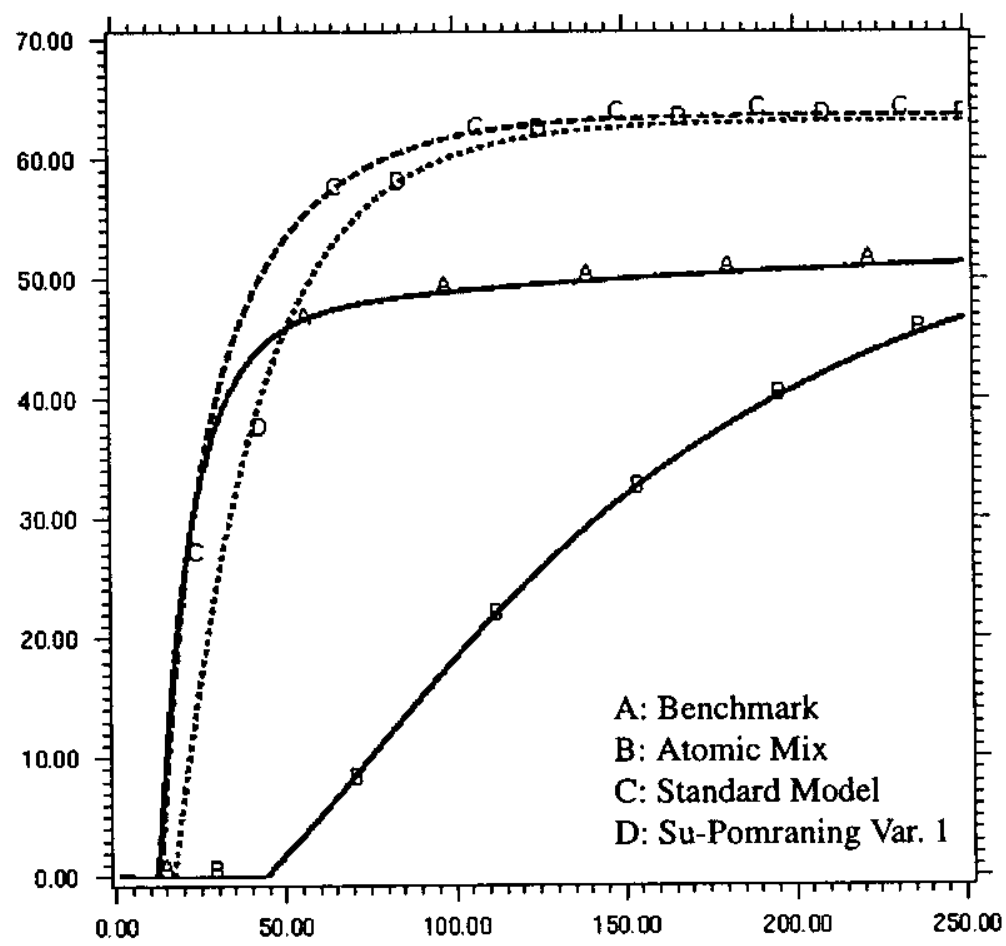

FIGURE 57. $\langle$ Trans $\rangle$ versus Time Step - Variable Opacities

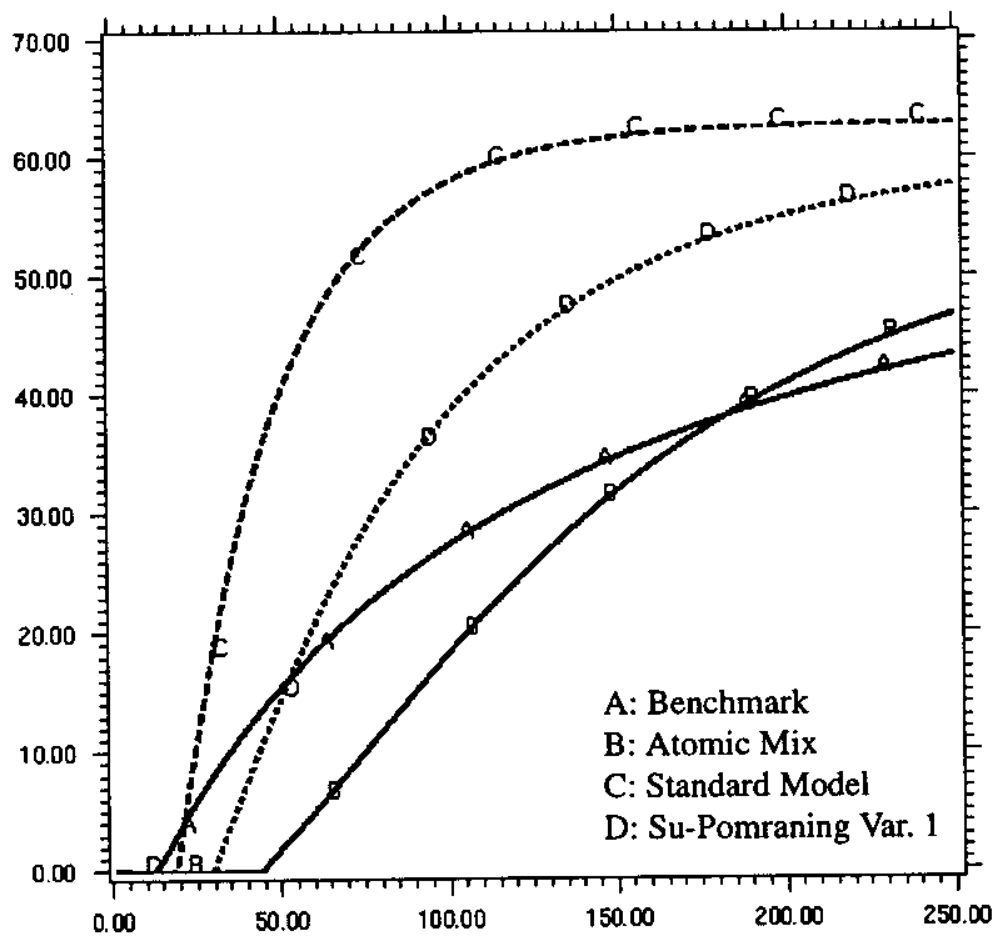


FIGURE 58. 〈Trans $\rangle$ versus Time Step - Variable Opacities

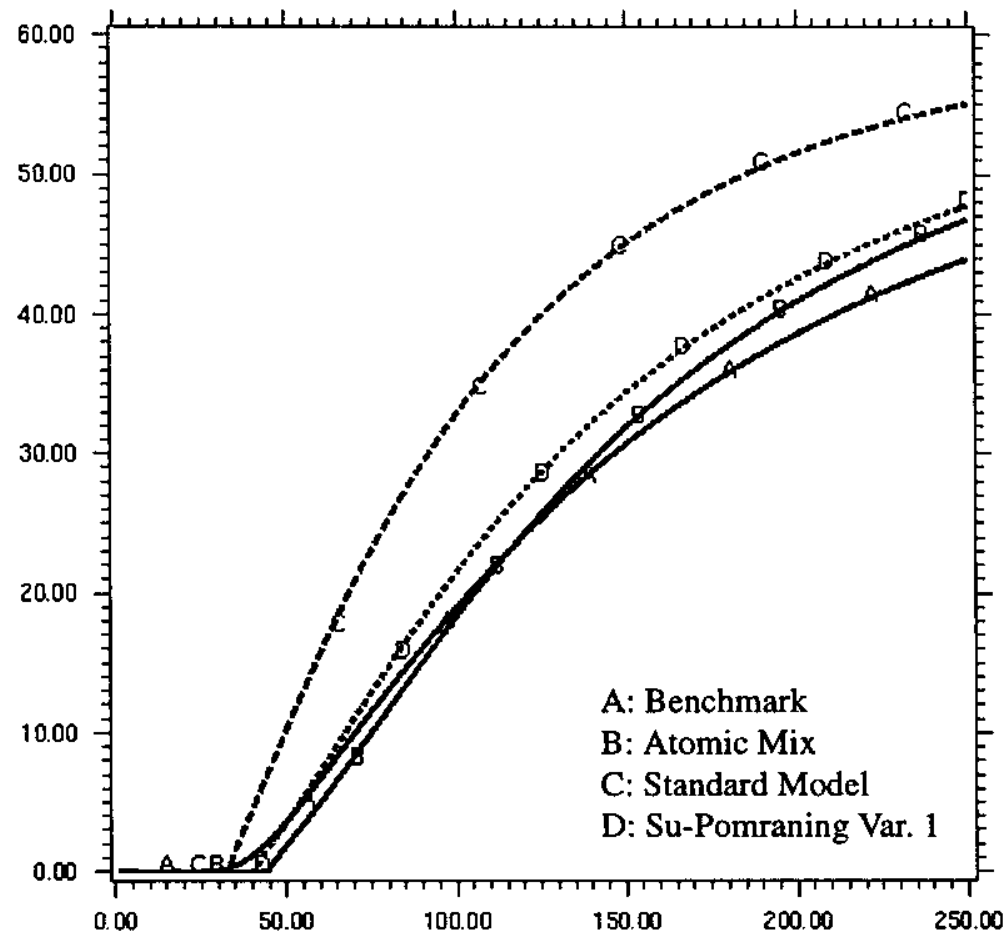

FIGURE 59. $\langle$ Trans $\rangle$ versus Time Step - Variable Opacities

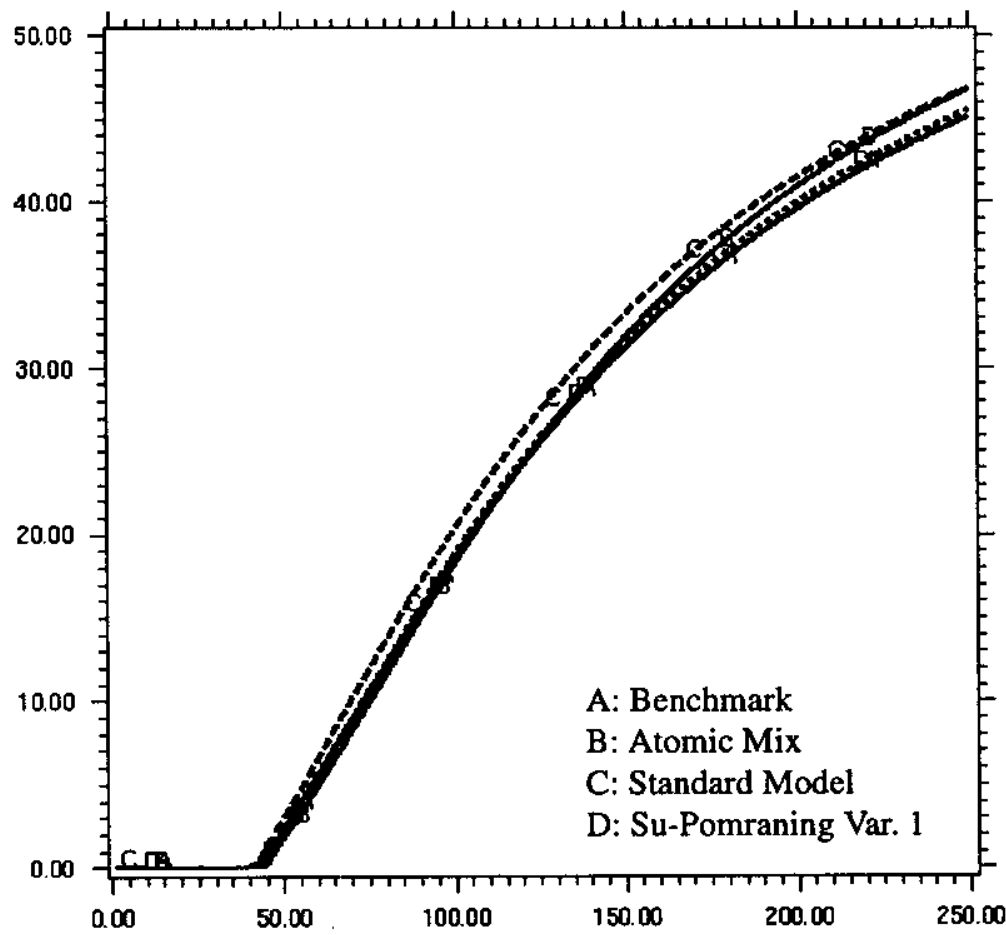


FIGURE 60. Benchmark Variances - Variable Opacities

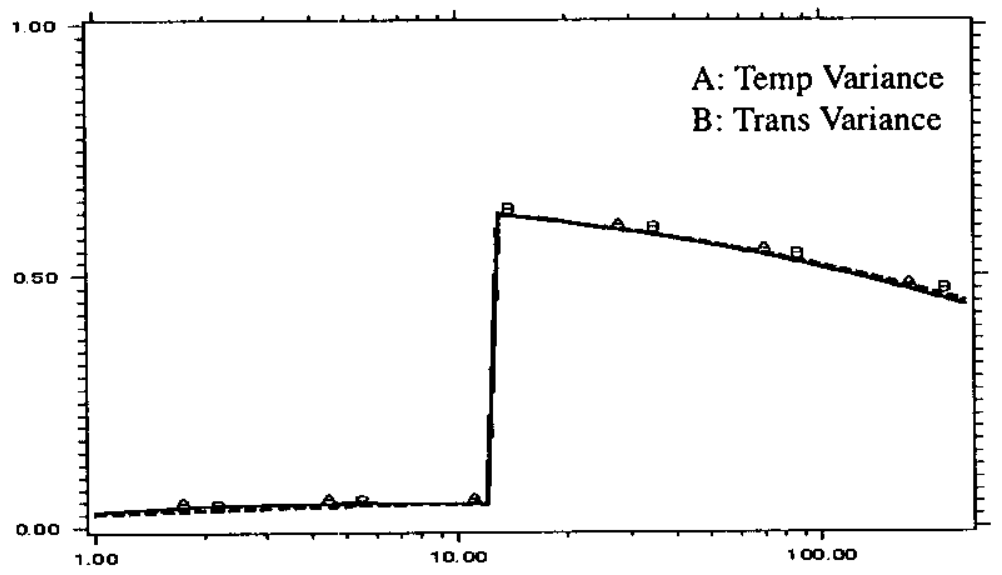

FIGURE 61. Benchmark Variances - Variable Opacities

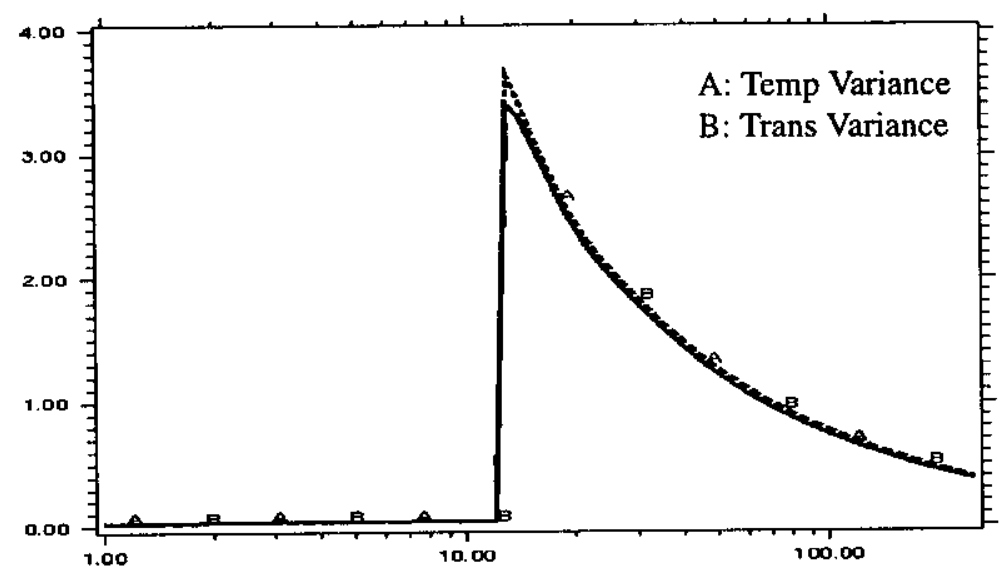

FIGURE 62. Benchmark Variances - Variable Opacities

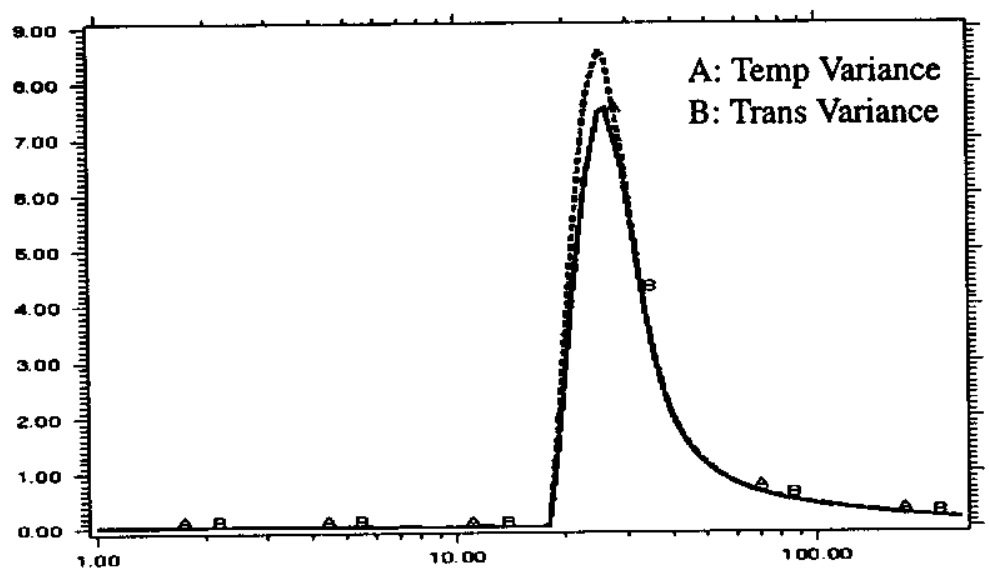


FIGURE 63. Benchmark Variances - Variable Opacities

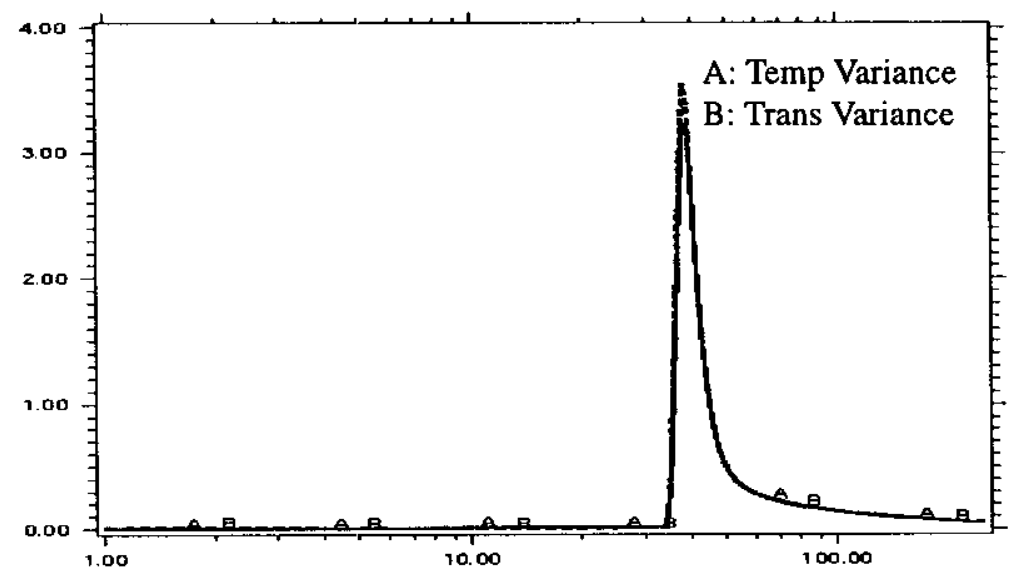

Once again, the introduction of new and strong correlations with the temperature dependent opacities wreak havoc with the solutions. This is exactly what happened with the 1-D slab geometry models and it should come as no surprise here. This is because the closure relations Eqs. (37) and (38) were both derived from the two state partitioning of the total possible set of statistical realizations, as described in Sec. (3.2). Neither coupling relation was designed to handle the correlations arising from continuously variable opacities.

Again the four methods are all converging to the atomic mix answer in the small correlation length limit. However it is difficult to see from the plots of $\langle\varphi\rangle$ and $\langle$ Trans $\rangle$ what is happening to the atomic mix model in this limit due to the errors involved. Clearly the problem has not evolved to steady state within the time frame of the runs, so it is difficult to judge the behavior of the models there either. Yet the convergent behavior is evident none the less. Again, this is a good check. It is however clear from the variances that the atomic mix answer is once again wrong for the transient period in the small correlation length limit. Hence the big hump once again seen in the variance there. 


\section{CONCLUSIONS}

The four main chapters of this thesis contain many results and they are summarized here.

\subsection{So What Was Learned In all This?}

\subsection{Atomic Mix Assumptions and Spatial Connectivity}

When it was decided to include the atomic mix formulation as one of the approximate methods to test, it was believed that the atomic mix was a built in check on the small correlation length limit behavior of any approximate model. It was believed that any proposed model should recover the same answer as the atomic mix formulation when the chunk sizes were shrunk to very small sizes. After all, this is the case for the standard model[11] and the Su-Pomraning variations[16] as they were originally developed, i.e for the radiation transport equation alone. So it was quite surprising when it was found that (for this problem) the approximate models and the benchmark converged in the small chunk limit, but that the atomic mix model did not. True, all the methods converged in the steady state for small chunks, but not in the transient. Clearly there was either an error in one of the solvers of there was something else going on. Eventually came an understanding of the following. The atomic mix formulation is a kind of spatial/volumetric averaging of the material properties. And this averaging is correct in the limit of small mixing only if the process being modeled has some kind of spatial smoothing in its formulation. In other words, the differential equation being modeled must have some spatial operator connecting the quantity through space. In formulating the equation for material temperature, ther- 
mal diffusion is neglected here. And hence there is no spatial derivative in that equation. The temperature equation is a purely local equation in space. This is why the atomic mix method was not getting the correct answer in the transient. It recovered the correct answer in the steady state only because of it's coupling to the transport equation, which does recover the atomic mix formulation in the small correlation length limit. Refer to the discussion in Sec. (9.3) for further analysis.

\subsection{Stability of Coupled Source Iteration}

When confronted with the task of numerically solving a differential equation, it is often much easier to develop a method then to determine the method's numerical stability. Analytic evaluation of a method's stability criteria is often very difficult, if possible at all. So it was a pleasant surprise to arrive at the stability criteria presented in Appendix (A). Not only does this result lend confidence in the use of a method, it can explain what is going on when the method fails - as was the case in Sec. (9.3) when the adaptation of the Supomraning model failed to converge after the idea of temperature coupling as an effective scattering was incorporated.

\subsection{The Role of Thermal Emission in Stochastic Transport}

In hindsight it is obvious that scattering and thermal emission of photons should play similar roles in the stochastic radiation transport process. But this was not at all clear when this project started. The initial formulations of the standard model as presented in Sec. (8.3) were meant to simulate the type of problem when the standard model gets the exact answer, but with a material temperature equation thrown in. It was not expected that this simple extension to change the exactness of the standard model formulation. But it did. In trying to understand why this was so, I came clear that the way scattering redirects pho- 
tons into other directions is much like the way thermal emission redirects photons into other directions. And for both processes, the act of computing the ensemble average of the radiation specific intensity (at any point) now means averaging over all past possible flight histories of the photon, in all directions. Without scattering or thermal emission, one need only average over the past flight on a single quadrature ray. It is this coupling of the average to the flight histories over all directions that explains why the standard model did not get the exact answer for the model in Sec. (8.3).

This led to the idea of treating the thermal emission as a kind of effective scattering-like process and the use of the Su-Pomraning model with effective scattering coefficient. This idea showed promise in the 1-D slab case by consistently improving over the standard model formulation. But this improvement was not there in the 1-D spherical case. It may well be that the generalization of the Su-Pomraning closure does not naively generalize to the 1-D spherical geometry. It was after all derived on the 1-D slab geometry, and it was adopted it here in an ad hoc way. It might be instructive to redo the Su-Pomraning derivation of their closure forms but in a 1-D spherical geometry, and see if the closure changes.

\subsection{Correlations from Temperature Dependent Opacities}

Whatever the performance of the various (non-benchmark) methods when used with constant opacities, all performed badly when the opacities were allowed to depend on material temperature. The standard model and the Su-Pomraning variations both depend on the idea that the total of all possible states of material distributions can be divided into two convenient partitions - either material $A$ or material $B$ is located at point $\vec{r}$. And that within these two partitions the value of the opacities are constant. If the opacities are not constant, one cannot compute averages involving the material opacities over the two parti- 
tions in a simple way. But this is exactly what the formulation of the standard model and the Su-Pomraning model depend on for their simplicity. They assume that the value of the opacities do not correlate to the value of anything else within the two natural partitions of the total set of allowable states. Hence, when the opacities are temperature dependent, all sorts of new strong correlations are ignored by the standard and Su-pomraning models.

This results in the large errors seen in Sec. (9.4) and Sec. (12.4).

\subsection{Usefulness of Two-Partition Restricted Average Operators}

The poor performance of the models based on coupled transport like equations (standard model and Su-Pomraning variations) when using temperature dependent opacities led me to the conclusion that two-partition restricted average operators (refer to Sec. (3.2)) may not be useful for treating problems with continuously variable stochastic coefficients. One really needs restricted average operators that average over all possible allowable values of the continuously variable stochastic coefficients. This leads not to two coupled transport-like equations (for example Eq. (21) ) but rather to an infinite number of coupled equations. For example this would replace the two equations represented by Eq. (21) with a set of equations - one equation for each value that $\sigma_{i}(T)$ could attain. Given the large number of resulting equations one would have to solve, the practical usefulness of this approach is questionable.

\subsection{Slab Geometry Models - General Comments}

The use of material temperature as an effective scattering showed significant improvement over the standard model, and it was much better generally than the atomic mix method also. The choice of chunk sizes demonstrates clearly that mix structure has an 
important role in the solution and that the atomic mix answer can be very wrong. The use of time dependence was important in that it brought out the limitations of the atomic mix approximation for uses in the small correlation length limit. With temperature dependent opacities, nothing compared well with the benchmark, although all methods showed convergence to the same steady state answer in the small chunk limits. The methods also clearly demonstrated the generally accepted behavior of over absorption by the atomic mix method[11] - something which was mysteriously lacking from the 1-D spherical runs.

\subsection{Spherical Geometry Models - General Comments}

The standard model without any material coupling did remarkably well in this geometry in spite of the fact that the mixing statistics are no longer HM. With the coupling to material temperature, the standard model and the Su-Pomraning variation with effective scattering did equally well (or badly as the case may be). Neither did any better than simple atomic mix in this case. This turnaround was quite surprising considering the success demonstrated by the standard model when material temperature was excluded.

It was also surprising to see that the atomic mix method consistently had higher steady state values than the other methods - except when the material temperature coupling was turned off. It was expected that the atomic mix model to achieve lower steady state values for all the runs, just as it did in the slab geometry runs. After all, it is a "well known" phenomena[11]. The fact that the atomic mix method only achieved lower steady state value with the material temperature coupling turned off and just the opposite when the material temperature coupling is turned on, leads me to suspect that this may have something to do with the geometry involved here. It is suspected that this "something' lies in the way that mixing regions can exchange photons across the core. This is a connectivity between parts 
of the mixing region which is not present in the slab geometry runs. However, if the only source of photons is in the core (as it was for all my tests), this connectivity only occurs if there is a redirection in angle for the photons. In other words if there is scattering or thermal emission. Otherwise the photons start out streaming outward from the core and just keep on going. They never get redirected back inward. So this means that this mixing region connectivity vanishes when there is no scattering or coupling to material temperature. Hence perhaps, the observed difference in relative behavior between the models when the material temperature coupling was absent versus when it was present

\subsection{Needed Improvements}

There is still much to be done. This work only scratched the surface of this field.

\subsection{Better Temperature Models}

The correlations introduced by continuously variable stochastic coefficients cannot be ignored. They must be accounted for if accurate solutions are to be obtained. Clearly, ignoring them as was done in deriving the methods using the two-partition restricted average operators and standard closure forms, gets the wrong answer when the opacities are allowed to vary with temperature. Many real problems will have variable stochastic coefficients and this problem will need to be addressed to treat them. This may well require an entire re-thinking of the problem.

\subsection{Other Statistics}

It appears that the use of the standard model (and others based on it) with mixing statis- 
tics that are not HM may yet provide useful results. It would be interesting to perform an entire series of tests on these models to see just how sensitive they are to variations in the mixing statistics. Can one just replace the average chunk size $\Lambda_{j}$ in Eq. (37) with the average for whatever statistics are used? how well would that work? It be interesting to find out. It certainly seemed to do fairly well in the 1-D sphere when temperature coupling was not present.

Regardless of the approach taken to the formulation, one needs to be able to treat more than just Poisson distributed materials if one hopes to model real life problems. One set of statistics of particular interest are Levy statistics. As mentioned earlier in the survey chapter, it has been suggested that Levy statistics are a much better representation of turbulent mixing in Rayleigh-Taylor instabilities[21], than say Poisson statistics. After all, the goal is to eventually make the connection between real problems and the stochastic models. And this means using real statistics - not dictating statistics of convenience to the problem. Therefore, methods for treating an arbitrary set of mixing statistics need to be developed and tested.

\subsection{Other Geometries}

Just as one would like to use a set of statistics appropriate to any problem at hand, the same is true for the geometry of the problem. Clearly, real problems will rarely conform themselves to conveniently fit within 1-D formulations. To treat real problems, models are needed that can be adapted to formulation and testing on the geometry at hand. The standard model can easily be formulated on any geometry. But because of the difficulties associated with generating computational meshes which are good examples of a given 
statistics, the benchmarks cannot generally be computed for comparison. However, given real experimental data this would not be necessary as one would then just compare to data.

\subsection{Comparison with Experiment}

Which brings us to an important point. I know of no examples in the literature where the various methods were compared to real experimental data. This kind of testing could provide invaluable insight into the various processes involved and the relative importance of the processes. Generating models is one thing, but getting them to work predicting real processes is another. The time is ripe for someone to take the theory and approximate models that have been developed in the literature over that past ten or fifteen years and examine their performance on a real stochastic media.

\subsection{Epilog}

When I first set out on this project, I knew little of the actual direction this work would take. It is part of the process, I suppose, that producing a doctoral dissertation involves a genuine exploration. But little did I expect to spent as much time in the act of searching for new ways to contribute to the field of stochastic transport as actually trying solve the problems. Stochastic transport is a very difficult problem in it's complete generality. And that I was able to make some contributions to help further it's understanding is gratifying. I learned much that I didn't know when I started this. And hopefully, it will help others take the process further. 


\section{APPENDIX A: Numerical Stability of Coupled Source}

\section{Iteration}

When the numerical solution of an equation (or set of equations) involves an iteration process, the successful solution requires that the iteration process be a stable one. In the case of the solution to discretized differential equations, one often is confronted with an iterative solution which can be written in matrix/vector form. Then the requirement of stability for the iterative linear system can be written in the following way. Let vector $\vec{x}_{n}$ be the solution after the nth iteration of the process

$$
M \vec{x}_{n}=N \vec{x}_{n-1}+\vec{b}
$$

where the $M$ and $N$ are matrices specific to the problem. Then the iteration Eq. (107) is stable if the spectral radius of the matrix $A=M^{-1} N$ is less than one. With this in mind, consider the numerical stability of a generalized class of coupled stochastic radiation transport equations.

The source-free equation of neutral particle transport for single energy group and constant opacities is in steady state

$$
\vec{\Omega} \cdot \nabla \psi(\grave{l}, \vec{\Omega})+\sigma \psi(\grave{r}, \vec{\Omega})=\frac{\sigma_{s}}{\theta} \int \psi d \vec{\Omega}^{\prime}
$$


where $\vec{\Omega}$ is the direction of particle travel, $\sigma$ is the total opacity, $\sigma_{s}$ is the scattering opacity, $\psi$ is the particle specific intensity, and $\theta=\int d \vec{\Omega}$ is a dimensionality constant. The direction integral is taken over all directions. When the background material is randomly mixed the total opacity $\sigma$ and the scattering opacity $\sigma_{s}$ are binary stochastic quantities known only via some statistical description. Hence the specific intensity $\psi(\vec{\lambda}, \vec{\Omega})$ is a continuous stochastic quantity. The goal then is to obtain, as the solution to this problem, the ensemble average of the specific intensity which is denoted as $\langle\psi\rangle$.

Consider here a general form for the solution of $\langle\psi\rangle$ based on a model due to Su and Pomraning, which is

$$
\vec{\Omega} \cdot \nabla \psi_{1}+\sigma_{1} \psi_{1}=\frac{\sigma_{s 1}}{\theta} \int \Psi_{1} d \vec{\Omega}^{\prime}+\frac{\kappa \psi_{2}}{\lambda_{2}(\vec{\Omega})}-\frac{\kappa \psi_{1}}{\lambda_{1}(\vec{\Omega})}+\frac{\eta \int \Psi_{1} d \vec{\Omega}^{\prime}}{\theta \lambda_{1}(\vec{\Omega})}-\frac{\eta \int \Psi_{2} d \vec{\Omega}^{\prime}}{\theta \lambda_{2}(\vec{\Omega})}
$$

and

$$
\vec{\Omega} \cdot \nabla \psi_{2}+\sigma_{2} \Psi_{2}=\frac{\sigma_{s 2}}{\theta} \int \Psi_{2} d \vec{\Omega}^{\prime}+\kappa \frac{\kappa \Psi_{1}}{\lambda_{1}(\vec{\Omega})}-\frac{\kappa \Psi_{2}}{\lambda_{2}(\vec{\Omega})}+\frac{\eta \int \Psi_{2} d \overrightarrow{\Omega^{\prime}}}{\theta \lambda_{2}(\vec{\Omega})}-\frac{\eta \int \Psi_{1} d \vec{\Omega}^{\prime}}{\theta \lambda_{1}(\vec{\Omega})}
$$

where the ensemble average of the specific intensity is then given by

$$
\langle\psi\rangle=\psi_{1}+\psi_{2}
$$

Here $\sigma_{i}$ and $\sigma_{s i}$ are the total and scattering opacities for material $i$ and $\lambda_{i}(\vec{\Omega})$ is the average size of a chunk of material $i$ as seen by a particle moving along the ray $\vec{\Omega}$. The $\mathrm{K}$ and 
$\eta$ are positive weighting constants. The $\sigma_{i}$ and $\sigma_{s i}$ are now constant and the problem has been reduced to solving two coupled deterministic transport-like equations.

A common numerical technique for the solution of Eq. (108) is known as Source Iteration. In this method the right hand side of Eq. (108) is held constant as the left hand side is inverted. The right hand side is then updated and the process is repeated. Denote the $n^{t h}$ iteration of the specific intensity as $\psi^{n}$. Then the method can be expressed as the following recursion,

$$
\grave{\Omega} \cdot \nabla \psi^{n+1}(\vec{r}, \grave{\Omega})+\sigma \psi^{n+1}(\vec{r}, \vec{\Omega})=\frac{\sigma_{s}}{\theta} \int \psi^{n} d \grave{\Omega}^{\prime}
$$

It can be shown that this technique is stable if

$$
c=\frac{\sigma_{s}}{\sigma}<1
$$

The number $c$ is called the scattering ratio and Eq. (113) is a standard result well known within the transport literature [22].

The Source Iteration technique applied to Eqs. (109) and (110) gives the coupled recursion

$$
\begin{aligned}
& \vec{\Omega} \cdot \nabla \psi_{1}^{n+1}+\sigma_{1} \psi_{1}^{n+1}+\frac{\kappa \psi_{1}^{n+1}}{\lambda_{1}(\vec{\Omega})}-\frac{\kappa \psi_{2}^{n+1}}{\lambda_{2}(\vec{\Omega})}=\frac{\sigma_{s 1}}{\theta} \int \psi_{1}^{n} d \vec{\Omega}^{\prime}+\frac{\eta \int \Psi_{1}^{n} d \bar{\Omega}^{\prime}}{\theta \lambda_{1}(\vec{\Omega})}-\frac{\eta \int \psi_{2}^{n} d \vec{\Omega}^{\prime}}{\theta \lambda_{2}(\vec{\Omega})} \\
& \vec{\Omega} \cdot \nabla \psi_{2}^{n+1}+\sigma_{2} \psi_{2}^{n+1}+\frac{\kappa \psi_{2}^{n+1}}{\lambda_{2}(\vec{\Omega})}-\frac{\kappa \Psi_{1}^{n+1}}{\lambda_{1}(\vec{\Omega})}=\frac{\sigma_{s 2}}{\theta} \int \psi_{2}^{n} d \vec{\Omega}^{\prime}+\frac{\eta \int \Psi_{2}^{n} d \vec{\Omega}^{\prime}}{\theta \lambda_{2}(\vec{\Omega})}-\frac{\eta \int \Psi_{1}^{n} d \vec{\Omega}^{\prime}}{\theta \lambda_{1}(\vec{\Omega})}
\end{aligned}
$$


It is shown that the stability criteria similar to Eq. (113) but generalized to the recursion Eqs. (114) and (115) in a 1-D slab geometry is

$$
\max \left[\frac{\sigma_{s 1}}{\sigma_{1}}, \frac{\sigma_{s 2}}{\sigma_{2}}, \frac{\eta}{\kappa}\right]=\max \left[c_{1}, c_{2}, \frac{\eta}{\kappa}\right]<1 .
$$

\subsection{Von Neumann Stability}

To evaluate the stability of the coupled recursion Eqs. (114) and (115), perform a Von Neumann stability analysis. This essentially means that if the problem is stable in Fourier space then it will be stable in position space as well.

But first, replace the direction integrals in Eqs. (114) and (115) with quadrature rule approximations. This is a common method called Discrete Ordinates [17] for the numerical solution of Eq. (108) and it is adopted here. This gives

$$
\int \Psi_{p} d \vec{\Omega}^{\prime}=\sum_{m=1}^{M} w_{m} \Psi_{p m}
$$

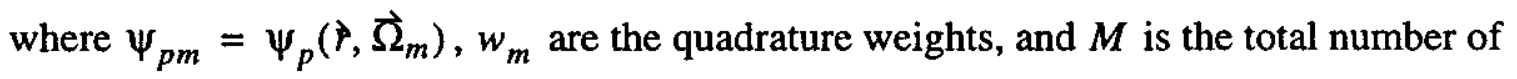
quadrature directions. Then $\dot{\varphi}_{p}$ is a vector of length $M$ with components $\psi_{p m}$ and the integral in Eq. (117) can be represented in the matrix-vector form 


$$
W \cdot \vec{\Psi}_{p}=\left[\begin{array}{c}
\int \Psi_{p} d \vec{\Omega}^{\prime} \\
: \\
\vdots \\
\int \Psi_{p} d \vec{\Omega}^{\prime}
\end{array}\right]
$$

where $W$ is the $M x M$ matrix

$$
W=\left[\begin{array}{ccccc}
w_{1} & w_{2} & \ldots & \ldots & w_{M} \\
w_{1} & w_{2} & \ldots & \ldots & w_{M} \\
: & & & & \vdots \\
: & & & & \vdots \\
w_{1} & w_{2} & \ldots & \ldots & w_{M}
\end{array}\right]
$$

Notice the matrix $W$ is rank 1 and has only one non-zero eigenvalue given by

$$
\Lambda_{W}=\sum_{m=1}^{M} w_{m}=\operatorname{tr}(W)
$$

Now transform to Fourier space. Make the ansatz that $\psi$ can be expanded in a discrete Fourier basis,

$$
\psi_{p m}=\sum_{A l l \mathrm{k}} \alpha_{p m}^{k} e^{i k \cdot \grave{x}}
$$

Then

$$
\grave{\Omega}_{m} \cdot \nabla \Psi_{p m}=\sum_{A l l \mathrm{k}} i\left(\grave{\Omega}_{m} \cdot \vec{k}\right) \alpha_{p m}^{k} e^{i k \cdot \vec{t}}
$$


where the recursion index is dropped for now. The expansion coefficients $\alpha_{p m}^{k}$ can now be isolated using the orthogonality of the expansion basis. First, substitute Eqs. (121) and (122) into Eqs. (114) and (115) to get

$$
\begin{aligned}
& \sum_{\text {All } k}\left(\left(i \vec{\Omega}_{m} \cdot \vec{k}+\sigma_{1}+\frac{\kappa}{\lambda_{1 m}}\right) \alpha^{k}{ }_{1 m}-\frac{\kappa \alpha_{2 m}^{k}}{\lambda_{2 m}}\right) e^{i \vec{k} \cdot \grave{l}}= \\
& \sum_{\text {All } k}\left(\sum_{l=1}^{M} w_{l}\left(\left(\frac{\sigma_{s 1}}{\theta}+\frac{\eta}{\theta \lambda_{1 m}}\right) \alpha_{1 l}^{k}-\frac{\eta}{\theta \lambda_{2 m}} \alpha^{k}\right)\right) e^{i \dot{k} \cdot \grave{l}}
\end{aligned}
$$

and

$$
\begin{aligned}
& \sum_{\text {All } k}\left(\left(i \vec{\Omega}_{m} \cdot \vec{k}+\sigma_{2}+\frac{\kappa}{\lambda_{2 m}}\right) \alpha^{k}{ }_{2 m}-\frac{\kappa \alpha_{1 m}^{k}}{\lambda_{1 m}}\right) e^{i k \cdot \vec{t}}=. \\
& \sum_{\text {All } k}\left(\sum_{l=1}^{M} w_{l}\left(\left(\frac{\sigma_{s 2}}{\theta}+\frac{\eta}{\theta \lambda_{2 m}}\right) \alpha_{2 l}^{k}-\frac{\eta}{\theta \lambda_{1 m}} \alpha_{1 l}^{k}\right)\right) e^{i k \cdot t}
\end{aligned}
$$

Multiplying both sides of Eqs. (123) and (124) by $e^{-i \vec{q} \cdot \hat{t}}$ and integrating over all $\vec{x}$ gives

$$
\left(i \vec{\Omega}_{m} \cdot \vec{q}+\sigma_{1}+\frac{\kappa}{\lambda_{1 m}}\right) \alpha^{q} 1 m-\frac{\kappa \alpha^{q} 2 m}{\lambda_{2 m}}=\left(\frac{\sigma_{s 1}}{\theta}+\frac{\eta}{\theta \lambda_{1 m}}\right) \sum_{l=1}^{M} w_{l} \alpha^{q} 1 l-\frac{\eta}{\theta \lambda_{2 m}} \sum_{l=1}^{M} w_{l} \alpha^{q} 2 l \text { (EQ }
$$

and

$$
\left(i \vec{\Sigma}_{m} \cdot \vec{q}+\sigma_{2}+\frac{\kappa}{\lambda_{2 m}}\right) \alpha^{q} 2 m-\frac{\kappa \alpha^{q} 1 m}{\lambda_{1 m}}=\left(\frac{\sigma_{s 2}}{\theta}+\frac{\eta}{\theta \lambda_{2 m}}\right) \sum_{l=1}^{M} w_{l} \alpha^{q} 2 l-\frac{\eta}{\theta \lambda_{1 m}} \sum_{l=1}^{M} w_{l} \alpha^{q} 1 l
$$


for all $q$. Relabel $q$ back to $k$. Let us denote $\Gamma_{m}=i \vec{\Omega}_{m} \cdot \vec{k}$ and drop the $k$ index on the $\alpha_{p m}^{k}$. Then, putting the index for the recursion iteration back in gives the Fourier component recursion equivalent to Eqs. (114) and (115),

$$
\left(\Gamma_{m}+\sigma_{1}+\frac{\kappa}{\lambda_{1 m}}\right) \alpha_{1 m}^{n+1}-\frac{\kappa}{\lambda_{2 m}} \alpha_{2 m}^{n+1}=\left(\frac{\sigma_{s 1}}{\theta}+\frac{\eta}{\theta \lambda_{1 m}}\right) \sum_{l=1}^{M} w_{l} \alpha^{n} 1 l-\frac{\eta}{\theta \lambda_{2 m}} \sum_{l=1}^{M} w_{l} \alpha^{n} 2 l \text { (EQ }
$$

and

$$
\left(\Gamma_{m}+\sigma_{2}+\frac{\kappa}{\lambda_{2 m}}\right) \alpha_{2 m}^{n+1}-\frac{\kappa}{\lambda_{1 m}} \alpha^{n+1}=\left(\frac{\sigma_{s 2}}{\theta}+\frac{\eta}{\theta \lambda_{2 m}}\right) \sum_{l=1}^{M} w_{l} \alpha^{n} 2 l-\frac{\eta}{\theta \lambda_{1 m}} \sum_{l=1}^{M} w_{l} \alpha^{n}{ }_{1 l} \text {. (EQ }
$$

Now write this in matrix form. Define the four $M x M$ matrices $D_{p}, O_{p}, Q_{p}$, and $R_{p}$ as the diagonal matrices with components

$$
\begin{gathered}
D_{p, m m}=\Gamma_{m}+\sigma_{p}+\frac{\kappa}{\lambda_{p m}}, \\
O_{p, m m}=\frac{\kappa}{\lambda_{p m}}, \\
Q_{p, m m}=\sigma_{s p}+\frac{\eta}{\lambda_{p m}}
\end{gathered}
$$

and

$$
R_{p, m m}=\frac{\eta}{\lambda_{p m}}
$$


Also define the vector $\dot{\alpha}_{p}$ as the vector of length $M$ with the components $\alpha_{p m}$. Then rewrite Eqs. (127) and (128) in matrix form as

$$
\left[\begin{array}{l}
\vec{\alpha}_{1} \\
\vec{\alpha}_{2}
\end{array}\right]^{n+1}=A \cdot\left[\begin{array}{l}
\vec{\alpha}_{1} \\
\vec{\alpha}_{2}
\end{array}\right]^{n}
$$

where the recursion matrix $A$ is the $2 M x 2 M$ matrix defined by

$$
A=\frac{1}{\theta}\left[\begin{array}{cc}
D_{1} & -O_{2} \\
-O_{1} & D_{2}
\end{array}\right]^{-1} \cdot\left[\begin{array}{cc}
Q_{1} W & -R_{2} W \\
-R_{1} W & Q_{2} W
\end{array}\right]
$$

The recursion given by Eqs. (114) and (115) will be stable if the spectral radius of $A$ is less than one. Denote the spectral radius of $A$ as $\rho(A)$. The next task is to compute $\rho(A)$.

\subsection{Computing the Spectral Radius}

To compute the spectral radius of $A$, begin by computing the eigenvalues of the matrix

A.

Theorem 1: There exist two non-zero eigenvalues of the matrix A given either by

$$
\Lambda_{ \pm}=\frac{\left(\Lambda_{1}+\Lambda_{4}\right)}{2} \pm \frac{1}{2} \sqrt{\left(\Lambda_{1}-\Lambda_{4}\right)^{2}+4\left(\Lambda_{2} \Lambda_{3}\right)} .
$$

or

$$
\Lambda_{ \pm}=\Lambda_{1}, \Lambda_{4}
$$

with 


$$
\begin{aligned}
& \Lambda_{1}=\frac{1}{\theta} \sum_{m=1}^{M} \frac{\left[\left(\Gamma_{m}+\sigma_{2}+\frac{\kappa}{\lambda_{2 m}}\right)\left(\sigma_{s 1}+\frac{\eta}{\lambda_{1 m}}\right)-\frac{\kappa \eta}{\lambda_{1 m} \lambda_{2 m}}\right] w_{m}}{\left(\Gamma_{m}+\sigma_{1}+\frac{\kappa}{\lambda_{1 m}}\right)\left(\Gamma_{m}+\sigma_{2}+\frac{\kappa}{\lambda_{2 m}}\right)-\left(\frac{\kappa^{2}}{\lambda_{1 m} \lambda_{2 m}}\right)} \\
& \Lambda_{2}=\frac{1}{\theta} \sum_{m=1}^{M} \frac{\left[\frac{\kappa}{\lambda_{2 m}}\left(\sigma_{s 2}+\frac{\eta}{\lambda_{2 m}}\right)-\frac{\eta}{\lambda_{2 m}}\left(\Gamma_{m}+\sigma_{2}+\frac{\kappa}{\lambda_{2 m}}\right)\right] w_{m}}{\left(\Gamma_{m}+\sigma_{1}+\frac{\kappa}{\lambda_{1 m}}\right)\left(\Gamma_{m}+\sigma_{2}+\frac{\kappa}{\lambda_{2 m}}\right)-\left(\frac{\kappa^{2}}{\lambda_{1 m} \lambda_{2 m}}\right)} \\
& \Lambda_{3}=\frac{1}{\theta} \sum_{m=1}^{M} \frac{\left[\frac{\kappa}{\lambda_{1 m}}\left(\sigma_{s 1}+\frac{\eta}{\lambda_{1 m}}\right)-\frac{\eta}{\lambda_{1 m}}\left(\Gamma_{m}+\sigma_{1}+\frac{\kappa}{\lambda_{1 m}}\right)\right] w_{m}}{\left(\Gamma_{m}+\sigma_{1}+\frac{\kappa}{\lambda_{1 m}}\right)\left(\Gamma_{m}+\sigma_{2}+\frac{\kappa}{\lambda_{2 m}}\right)-\left(\frac{\kappa^{2}}{\lambda_{1 m} \lambda_{2 m}}\right)} \\
& \Lambda_{4}=\frac{1}{\theta} \sum_{m=1}^{M} \frac{\left[\left(\Gamma_{m}+\sigma_{1}+\frac{\kappa}{\lambda_{1 m}}\right)\left(\sigma_{s 2}+\frac{\eta}{\lambda_{2 m}}\right)-\frac{\kappa \eta}{\lambda_{1 m} \lambda_{2 m}}\right] w_{m}}{\left(\Gamma_{m}+\sigma_{1}+\frac{\kappa}{\lambda_{1 m}}\right)\left(\Gamma_{m}+\sigma_{2}+\frac{\kappa}{\lambda_{2 m}}\right)-\left(\frac{\kappa^{2}}{\lambda_{1 m} \lambda_{2 m}}\right)} .
\end{aligned}
$$

Proof: Make use of the fact that the matrices $D_{1}, D_{2}, O_{1}$, and $O_{2}$ are all diagonal, and hence commute with each other, to evaluate the inverse matrix in Eq. (134) giving

$$
A=\frac{1}{\theta}\left[\begin{array}{cc}
{\left[D_{1} D_{2}-O_{1} O_{2}\right]^{-1}} & 0 \\
0 & {\left[D_{1} D_{2}-O_{1} O_{2}\right]^{-1}}
\end{array}\right] \cdot\left[\begin{array}{cc}
D_{2} & O_{2} \\
O_{1} & D_{1}
\end{array}\right] \cdot\left[\begin{array}{cc}
Q_{1} W & -R_{2} W \\
-R_{1} W & Q_{2} W
\end{array}\right]
$$

Define $\chi$ as the $M x M$ diagonal matrix $\chi=\left[D_{1} D_{2}-O_{1} O_{2}\right]^{-1}$ with components 


$$
\chi_{m m}=\frac{1}{\left(\Gamma_{m}+\sigma_{1}+\frac{\kappa}{\lambda_{1 m}}\right)\left(\Gamma_{m}+\sigma_{2}+\frac{\kappa}{\lambda_{2 m}}\right)-\left(\frac{\kappa^{2}}{\lambda_{1 m} \lambda_{2 m}}\right)}
$$

Then the matrix $A$ can be written

$$
A=\frac{1}{\theta}\left[\begin{array}{ll}
\chi\left(D_{2} Q_{1}-O_{2} R_{1}\right) W & \chi\left(O_{2} Q_{2}-R_{2} D_{2}\right) W \\
\chi\left(O_{1} Q_{1}-R_{1} D_{1}\right) W & \chi\left(D_{1} Q_{2}-O_{1} R_{2}\right) W
\end{array}\right] .
$$

Define the four $M x M$ sub-block matrices

$$
\begin{aligned}
& A_{1}=\frac{1}{\theta} \chi\left(D_{2} Q_{1}-O_{2} R_{1}\right) W, \\
& A_{2}=\frac{1}{\theta} \chi\left(O_{2} Q_{2}-R_{2} D_{2}\right) W, \\
& A_{3}=\frac{1}{\theta} \chi\left(O_{1} Q_{1}-R_{1} D_{1}\right) W, \\
& A_{4}=\frac{1}{\theta} \chi\left(D_{1} Q_{2}-O_{1} R_{2}\right) W,
\end{aligned}
$$

so that

$$
A=\left[\begin{array}{ll}
A_{1} & A_{2} \\
A_{3} & A_{4}
\end{array}\right]
$$

Define the $2 M$ eigenvalues of the matrix $A$ as $\Lambda$. The $\Lambda$ can be found using the characteristic equation for $A$. In other words by solving 


$$
\operatorname{Det}\left[\begin{array}{cc}
A_{1}-I \Lambda & A_{2} \\
A_{3} & A_{4}-I \Lambda
\end{array}\right]=0
$$

for the values $\Lambda$.

First consider the case where the $\Lambda$ cannot be one of the eigenvalues of $A_{1}$ or $A_{4}$. Factoring the determinant gives

$$
\operatorname{Det}\left[\begin{array}{cc}
\left(A_{1}-I \Lambda\right) & 0 \\
A_{3} & \left(A_{4}-I \Lambda\right)
\end{array}\right] \operatorname{Det}\left[\begin{array}{cc}
I & \left(A_{1}-I \Lambda\right)^{-1} A_{2} \\
0 & I-\left(A_{4}-I \Lambda\right)^{-1} A_{3}\left(A_{1}-I \Lambda\right)^{-1} A_{2}
\end{array}\right]=0 .(\text { EQ 150) }
$$

Using the fact that the determinant of block triangular matrices is the product of the determinants of the diagonal blocks, Eq. (150) becomes

$$
\operatorname{Det}\left[A_{1}-I \Lambda\right] \operatorname{Det}\left[A_{4}-I \Lambda\right] \operatorname{Det}\left[I-\left(A_{4}-I \Lambda\right)^{-1} A_{3}\left(A_{1}-I \Lambda\right)^{-1} A_{2}\right]=0 .
$$

The determinant of a matrix is just the product of its eigenvalues so

$$
\begin{aligned}
& \operatorname{Det}\left[A_{1}-I \Lambda\right]=\prod_{i=1}^{M}\left(\Lambda_{1 i}-\Lambda\right), \\
& \operatorname{Det}\left[A_{4}-I \Lambda\right]=\prod_{j=1}^{M}\left(\Lambda_{4 j}-\Lambda\right)
\end{aligned}
$$

and

$$
\operatorname{Det}\left[I-\left(A_{4}-I \Lambda\right)^{-1} A_{3}\left(A_{1}-I \Lambda\right)^{-1} A_{2}\right]=\prod_{k=1}^{M}\left(1-f_{k}(\Lambda)\right)
$$


where $\Lambda_{1 i}$ and $\Lambda_{4 i}$ are the $i$ th eigenvalues of $A_{1}$ and $A_{4}$, and the $f_{k}(\Lambda)$ are the $k$ th eigenvalues of the matrix $\left(A_{4}-I \Lambda\right)^{-1} A_{3}\left(A_{1}-I \Lambda\right)^{-1} A_{2}$ and are functions of $\Lambda$. Then the eigenvalues of the matrix $A$ are given by the roots of

$$
\prod_{i=1}^{M}\left(\Lambda_{1 i}-\Lambda\right) \prod_{j=1}^{M}\left(\Lambda_{4 j}-\Lambda\right) \prod_{k=1}^{M}\left(1-f_{k}\right)=0
$$

At this point, Eq. (155) can be simplified by exploiting the properties of the sub-block matrices. Note that all four of the sub-block matrices have the form of a diagonal matrix times the matrix $W$. This means that each of the sub-block matrices have one and only one linearly independent row and hence only one non-zero eigenvalue. This one non-zero eigenvalue is easily found to just be the trace of the matrix. Denote the one non-zero eigenvalue of the sub-block matrix $A_{i}$ as $\Lambda_{i}=\operatorname{tr}\left(A_{i}\right)$, then Eq. (155) becomes

$$
\left(\Lambda-\Lambda_{1}\right)\left(\Lambda-\Lambda_{4}\right) \prod_{k=1}^{M}\left(1-f_{k}\right)=0 .
$$

The values $f_{k}$ are found by further making use of the properties of the $A_{i}$ sub-blocks. Consider that all the $A_{i}$ matrices have the form of products of a diagonal matrix, which is labeled $\Delta_{i}$, and the matrix $W$. So that in general

$$
A_{i}=\Delta_{i} W
$$

Denote the eigenvector of $A_{i}$ corresponding to it's single non-zero eigenvalue $\Lambda_{i}$, as $\vec{x}_{i}$, so that 


$$
A_{i} \cdot \vec{x}_{i}=\Delta_{i} W \cdot \vec{x}_{i}=\Lambda_{i} \vec{x}_{i}
$$

Also denote the eigenvector which corresponds to the one non-zero eigenvalue of $W$ as $\hat{x}$. It is easily seen by demonstration that $\hat{x}$ is just the vector of length $M$ comprised of all 1's.

$$
\hat{x}=\left[\begin{array}{c}
1 \\
: \\
: \\
\vdots \\
1
\end{array}\right]
$$

Then it is also easily seen that the eigenvectors $\vec{x}_{i}$ are just

$$
\grave{x}_{i}=\Delta_{i} \cdot \hat{x}
$$

Now multiply Eq. (158) through by the inverse of $\Delta_{i}$. Using Eq. (160) gives

$$
W \cdot \vec{x}_{i}=\Lambda_{i} \hat{x}
$$

Multiplying Eq. (161) through by $\Delta_{k}$ and using Eq. (160) again gives the rule that

$$
A_{k} \cdot \vec{x}_{i}=\Lambda_{i} \vec{x}_{k}
$$

Moreover, because the sub-block matrices are all of rank one, one has the general property that taking the dot product of the $A_{j}$ sub-block onto an arbitrary vector $\vec{y}$ with components $y_{k}$ the resulting product must lie in the space spanned by the single eigenvector $\vec{x}_{j}$. 
In other words

$$
A_{j} \cdot \grave{y}=C \grave{x}_{j}
$$

where $C$ is a constant given by

$$
C=\sum_{k=1}^{M} w_{k} y_{k} .
$$

To compute the $f_{k}$ one more rule is needed. Multiply the vector $\vec{x}_{i}$ by the matrix $\left(A_{k}-I \Lambda\right)$ and use Eq. (162). This gives

$$
\left(A_{k}-I \Lambda\right) \cdot \vec{x}_{i}=\Lambda_{i} \vec{x}_{k}-\Lambda \vec{x}_{i}
$$

Inverting $\left(A_{k}-I \Lambda\right)$ gives

$$
\vec{x}_{i}=\frac{\Lambda_{i} \vec{x}_{k}}{\left(\Lambda_{k}-\Lambda\right)}-\Lambda\left(A_{k}-I \Lambda\right)^{-1} \cdot \vec{x}_{i}
$$

Rearranging Eq. (166) gives the other needed identity

$$
\left(A_{k}-I \Lambda\right)^{-1} \cdot \vec{x}_{i}=\frac{\Lambda_{i} \vec{x}_{k}}{\Lambda\left(\Lambda_{k}-\Lambda\right)}-\frac{\vec{x}_{i}}{\Lambda}
$$

Now get the eigenvalues of the matrix $\left[\left(A_{4}-I \Lambda\right)^{-1} A_{3}\left(A_{1}-I \Lambda\right)^{-1} A_{2}\right]$. Take the dot product of this matrix onto an arbitrary vector $џ$, and using Eqs. (162) through (164) and Eq. (167) gives 


$$
\left[\left(A_{4}-I \Lambda\right)^{-1} A_{3}\left(A_{1}-I \Lambda\right)^{-1} A_{2}\right] \cdot \dot{y}=\frac{\Lambda_{2} C}{\Lambda\left(\Lambda_{1}-\Lambda\right)}\left[\frac{\Lambda_{3}}{\left(\Lambda_{4}-\Lambda\right)} \vec{x}_{4}-\vec{x}_{3}\right]
$$

Using a different $y$ vector will only change the value of the constant $C$. Clearly then the matrix $\left[\left(A_{4}-I \Lambda\right)^{-1} A_{3}\left(A_{1}-I \Lambda\right)^{-1} A_{2}\right]$ is also of rank one and possesses but a single nonzero eigenvalue. Then

$$
\grave{y}=\vec{x}_{4}-\frac{\left(\Lambda_{4}-\Lambda\right)}{\Lambda_{3}} \vec{x}_{3}
$$

is the eigenvector corresponding to the single non-zero eigenvalue

$$
f=\frac{\Lambda_{2} \Lambda_{3}}{\left(\Lambda_{1}-\Lambda\right)\left(\Lambda_{4}-\Lambda\right)}
$$

Now Eq. (156) reduces to

$$
\left(\Lambda-\Lambda_{1}\right)\left(\Lambda-\Lambda_{4}\right)\left(1-\frac{\Lambda_{2} \Lambda_{3}}{\left(\Lambda_{1}-\Lambda\right)\left(\Lambda_{4}-\Lambda\right)}\right)=0
$$

and one has that the two non-zero eigenvalues of the matrix $A$ are given from the roots of the quadratic

$$
\Lambda^{2}-\Lambda\left(\Lambda_{1}+\Lambda_{4}\right)+\left(\Lambda_{1} \Lambda_{4}-\Lambda_{2} \Lambda_{3}\right)=0
$$

which are explicitly

$$
\Lambda_{ \pm}=\frac{\left(\Lambda_{1}+\Lambda_{4}\right)}{2} \pm \frac{1}{2} \sqrt{\left(\Lambda_{1}-\Lambda_{4}\right)^{2}+4\left(\Lambda_{2} \Lambda_{3}\right)}
$$

when $\Lambda_{2}$ or $\Lambda_{3}$ are not zero. 
Now consider the case where the $\Lambda$ are allowed to be either $\Lambda_{1}$ or $\Lambda_{4}$. In this case the factoring of the determinant in Eq. (150) is not allowed. First notice that in general the matrix $A$ has at most two non-zero eigenvalues. This can easily be seen by taking the dot product of $A$ onto an arbitrary vector $[\vec{p} \phi]^{t}$ and using Eq. (163) to get

$$
\left[\begin{array}{ll}
A_{1} & A_{2} \\
A_{3} & A_{4}
\end{array}\right] \cdot\left[\begin{array}{l}
\vec{p} \\
\vec{q}
\end{array}\right]=C_{p}\left[\begin{array}{l}
\vec{x}_{1} \\
\vec{x}_{3}
\end{array}\right]+C_{q}\left[\begin{array}{l}
\vec{x}_{2} \\
\vec{x}_{4}
\end{array}\right]
$$

where the constants $C_{p}$ and $C_{q}$ are given by Eq. (164). Clearly the matrix $A$ maps any vector into the space spanned by the two vectors $\left[\begin{array}{ll}\vec{x}_{1} & \vec{x}_{3}\end{array}\right]^{t}$ and $\left[\vec{x}_{2} \vec{x}_{4}\right]^{t}$. Then it follows that $A$ can have at most two non-zero eigenvalues. And since the sum of the eigenvalues of $A$ is just the trace of $A$, i.e.

$$
\operatorname{tr}(A)=\operatorname{tr}\left(A_{1}\right)+\operatorname{tr}\left(A_{4}\right)=\Lambda_{1}+\Lambda_{4}
$$

it follows that if one of the eigenvalues of $A$ is $\Lambda_{1}$ the other must be $\Lambda_{4}$, and vice versa, giving

$$
\Lambda_{ \pm}=\Lambda_{1}, \Lambda_{4}
$$

Thus we have the result

$$
\Lambda_{ \pm}=\frac{\left(\Lambda_{1}+\Lambda_{4}\right)}{2} \pm \frac{1}{2} \sqrt{\left(\Lambda_{1}-\Lambda_{4}\right)^{2}+4\left(\Lambda_{2} \Lambda_{3}\right)} \quad \text { or } \quad \Lambda_{1}, \Lambda_{4}
$$

Combining the definitions of the sub-block matrices with the fact that $\Lambda_{i}=\operatorname{tr}\left(A_{i}\right)$ 
gives

$$
\begin{aligned}
& \Lambda_{1}=\frac{1}{\theta} \sum_{m=1}^{M} \frac{\left[\left(\Gamma_{m}+\sigma_{2}+\frac{\kappa}{\lambda_{2 m}}\right)\left(\sigma_{s 1}+\frac{\eta}{\lambda_{1 m}}\right)-\frac{\kappa \eta}{\lambda_{1 m} \lambda_{2 m}}\right] w_{m}}{\left(\Gamma_{m}+\sigma_{1}+\frac{\kappa}{\lambda_{1 m}}\right)\left(\Gamma_{m}+\sigma_{2}+\frac{\kappa}{\lambda_{2 m}}\right)-\left(\frac{\kappa}{\lambda_{1 m} \lambda_{2 m}}\right)} \\
& \Lambda_{2}=\frac{1}{\theta} \sum_{m=1}^{M} \frac{\left[\frac{\kappa}{\lambda_{2 m}}\left(\sigma_{s 2}+\frac{\eta}{\lambda_{2 m}}\right)-\frac{\eta}{\lambda_{2 m}}\left(\Gamma_{m}+\sigma_{2}+\frac{\kappa}{\lambda_{2 m}}\right)\right] w_{m}}{\left(\Gamma_{m}+\sigma_{1}+\frac{\kappa}{\lambda_{1 m}}\right)\left(\Gamma_{m}+\sigma_{2}+\frac{\kappa}{\lambda_{2 m}}\right)-\left(\frac{\kappa}{\lambda_{1 m} \lambda_{2 m}}\right)} \\
& \Lambda_{3}=\frac{1}{\theta} \sum_{m=1}^{M} \frac{\left[\frac{\kappa}{\lambda_{1 m}}\left(\sigma_{s 1}+\frac{\eta}{\lambda_{1 m}}\right)-\frac{\eta}{\lambda_{1 m}}\left(\Gamma_{m}+\sigma_{1}+\frac{\kappa}{\lambda_{1 m}}\right)\right] w_{m}}{\left(\Gamma_{m}+\sigma_{1}+\frac{\kappa}{\lambda_{1 m}}\right)\left(\Gamma_{m}+\sigma_{2}+\frac{\kappa}{\lambda_{2 m}}\right)-\left(\frac{\kappa}{\lambda_{1 m} \lambda_{2 m}}\right)} \\
& \Lambda_{4}=\frac{1}{\theta} \sum_{m=1}^{M} \frac{\left[\left(\Gamma_{m}+\sigma_{1}+\frac{\kappa}{\lambda_{1 m}}\right)\left(\sigma_{s 2}+\frac{\eta}{\lambda_{2 m}}\right)-\frac{\kappa \eta}{\lambda_{1 m} \lambda_{2 m}}\right] w_{m}}{\left[\left(\sigma_{1}+\frac{\kappa}{\lambda_{1 m}}\right)\left(\Gamma_{m}+\sigma_{2}+\frac{\kappa}{\lambda_{2 m}}\right)-\left(\frac{\kappa^{2}}{\lambda_{1 m} \lambda_{2 m}}\right)\right.}
\end{aligned}
$$

proving Theorem 1. $Q E D$.

Ultimately, the goal is to compute the spectral radius of the matrix $A$ which is the largest eigenvalue in absolute value, so that

$$
\rho(A)=\max \left[\left|\Lambda_{+}\right|,\left|\Lambda_{-}\right|\right]
$$

Given the complicated form of the eigenvalues of the sub-blocks and the form of Eq. (173), this looks to be a difficult task. To make things a bit easier, consider the problem in 
a 1-D slab geometry.

In a 1-D slab geometry the $\lambda_{i m}$ become

$$
\lambda_{i m}=\frac{\lambda_{i}}{\left|\mu_{m}\right|}
$$

where $\mu_{m}$ is the cosine of the angle the quadrature ray $\vec{\Omega}_{m}$ makes with the axis of symmetry. Then the $\Lambda_{i}$ become

$$
\begin{aligned}
& \Lambda_{1}=\frac{1}{\theta} \sum_{m=1}^{M} \frac{\left[\left(i \mu_{m} k+\sigma_{2}+\frac{\left|\mu_{m}\right| \kappa}{\lambda_{2}}\right)\left(\sigma_{s 1}+\frac{\left|\mu_{m}\right| \eta}{\lambda_{1}}\right)-\frac{\left|\mu_{m}\right|^{2} \kappa \eta}{\lambda_{1} \lambda_{2}}\right] w_{m}}{\left(i \mu_{m} k+\sigma_{1}+\frac{\left|\mu_{m}\right| \kappa}{\lambda_{1}}\right)\left(i \mu_{m} k+\sigma_{2}+\frac{\left|\mu_{m}\right| \kappa}{\lambda_{2}}\right)-\left(\frac{\left|\mu_{m}\right|^{2} \kappa^{2}}{\lambda_{1} \lambda_{2}}\right)} \\
& \Lambda_{2}=\frac{1}{\theta} \sum_{m=1}^{M} \frac{\left[\frac{\left|\mu_{m}\right| \kappa}{\lambda_{2}}\left(\sigma_{s 2}+\frac{\left|\mu_{m}\right| \eta}{\lambda_{2}}\right)-\frac{\left|\mu_{m}\right| \eta}{\lambda_{2}}\left(i \mu_{m} k+\sigma_{2}+\frac{\left|\mu_{m}\right| \kappa}{\lambda_{2}}\right)\right] w_{m}}{\left(i \mu_{m} k+\sigma_{1}+\frac{\left|\mu_{m}\right| \kappa}{\lambda_{1}}\right)\left(i \mu_{m} k+\sigma_{2}+\frac{\left|\mu_{m}\right| \kappa}{\lambda_{2}}\right)-\left(\frac{\left|\mu_{m}\right|^{2} \kappa^{2}}{\lambda_{1} \lambda_{2}}\right)} \\
& \Lambda_{3}=\frac{1}{\theta} \sum_{m=1}^{M} \frac{\left[\frac{\left|\mu_{m}\right| \kappa}{\lambda_{1}}\left(\sigma_{s 1}+\frac{\left|\mu_{m}\right| \eta}{\lambda_{1}}\right)-\frac{\left|\mu_{m}\right| \eta}{\lambda_{1}}\left(i \mu_{m} k+\sigma_{1}+\frac{\left|\mu_{m}\right| \kappa}{\lambda_{1}}\right)\right] w_{m}}{\left(i \mu_{m} k+\sigma_{1}+\frac{\left|\mu_{m}\right| \kappa}{\lambda_{1}}\right)\left(i \mu_{m} k+\sigma_{2}+\frac{\left|\mu_{m}\right| \kappa}{\lambda_{2}}\right)-\left(\frac{\left|\mu_{m}\right|^{2} \kappa^{2}}{\lambda_{1} \lambda_{2}}\right)} \\
& \Lambda_{4}=\frac{1}{\theta} \sum_{m=1}^{M} \frac{\left[\left(i \mu_{m} k+\sigma_{1}+\frac{\left|\mu_{m}\right| \kappa}{\lambda_{1}}\right)\left(\sigma_{s 2}+\frac{\left|\mu_{m}\right| \eta}{\lambda_{2}}\right)-\frac{\left|\mu_{m}\right|^{2} \kappa \eta}{\lambda_{1} \lambda_{2}}\right] w_{m}}{\left(i \mu_{m} k+\sigma_{1}+\frac{\left|\mu_{m}\right| \kappa}{\lambda_{1}}\right)\left(i \mu_{m} k+\sigma_{2}+\frac{\left|\mu_{m}\right| \kappa}{\lambda_{2}}\right)-\left(\frac{\left|\mu_{m}\right|^{2} \kappa^{2}}{\lambda_{1} \lambda_{2}}\right)}
\end{aligned}
$$


Calculation of Eq. (182) is complicated by the fact that the maximum is taken over all possible Fourier components $k$. There is however good evidence suggesting that the spectral radius is in fact dominated by the $k=0$ mode. It is easy to see that this is true for the simpler case of ordinary source iteration Eq. (112). Performing the Von Neumann analysis in slab geometry on Eq. (112) gives the recursion equation

$$
\left(i \mu_{m} k+\sigma\right) \alpha^{n+1}{ }_{m}=\frac{\sigma_{s}}{2} \sum_{l=1}^{M} w_{l} \alpha_{l}^{n} .
$$

Label the single non-zero eigenvalue of the resulting recursion matrix as $L$. Then the spectral radius of this recursion is the largest absolute value of $L$. Explicitly

$$
L=\frac{\sigma_{s}}{2} \sum_{l=1}^{M} \frac{{ }^{w} l}{\left(i \mu_{l} k+\sigma\right)} .
$$

Taking the absolute value of Eq. (189) gives

$$
|L|=\frac{\sigma_{s}}{2}\left[\left(\sum_{l=1}^{M} \frac{{ } \sigma}{\left(\mu_{l} k\right)^{2}+\sigma^{2}}\right)^{2}+\left(\sum_{l=1}^{M} \frac{w_{l} \mu_{l} k}{\left(\mu_{l} k\right)^{2}+\sigma^{2}}\right)^{2}\right]^{\frac{1}{2}} .
$$

With a Gaussian quadrature rule, the second sum in Eq. (190) is identically zero. Clearly then $|L|$ has the maximum at $k=0$ and Eq. (190) reduces to $|L|=\frac{\sigma_{s}}{\sigma}$. In light of the evidence, make the assumption that only the $k=0$ mode need be considered for computing Eq. (182). 
Theorem 2: In a 1-D slab geometry and assuming the $k=0$ mode, then

$$
\rho(A)=\max \left[\left|\Lambda_{+}\right|,\left|\Lambda_{-}\right|\right] \leq \max \left[\frac{\sigma_{s 1}}{\sigma_{1}}, \frac{\sigma_{s 2}}{\sigma_{2}}, \frac{\eta}{\kappa}\right]
$$

in the limit of an exact quadrature rule.

Proof:

With $k=0$, Eqs. (184) through (187) become

$$
\begin{gathered}
\Lambda_{1}=\frac{1}{2} \sum_{m=1}^{M} \frac{\left[\sigma_{s 1} \sigma_{2}+\left|\mu_{m}\right|\left(\kappa \frac{\sigma_{s 1}}{\lambda_{2}}+\eta \frac{\sigma_{2}}{\lambda_{1}}\right)\right] w_{m}}{\left[\sigma_{1} \sigma_{2}+\kappa\left|\mu_{m}\right|\left(\frac{\sigma_{1}}{\lambda_{2}}+\frac{\sigma_{2}}{\lambda_{1}}\right)\right]} \\
\Lambda_{2}=\frac{1}{2} \sum_{m=1}^{M} \frac{\left[\frac{\left|\mu_{m}\right|}{\lambda_{2}}\left(\kappa \sigma_{s 2}-\eta \sigma_{2}\right)\right] w_{m}}{\left[\sigma_{1} \sigma_{2}+\kappa\left|\mu_{m}\right|\left(\frac{\sigma_{1}}{\lambda_{2}}+\frac{\sigma_{2}}{\lambda_{1}}\right)\right]} \\
\Lambda_{3}=\frac{1}{2} \sum_{m=1}^{M} \frac{\left[\frac{\left|\mu_{m}\right|}{\lambda_{1}}\left(\kappa \sigma_{s 1}-\eta \sigma_{1}\right)\right] w_{m}}{\left[\sigma_{1} \sigma_{2}+\kappa\left|\mu_{m}\right|\left(\frac{\sigma_{1}}{\lambda_{2}}+\frac{\sigma_{2}}{\lambda_{1}}\right)\right]} \\
\Lambda_{4}=\frac{1}{2} \sum_{m=1}^{M} \frac{\left[\sigma_{s 2} \sigma_{1}+\left|\mu_{m}\right|\left(\kappa \frac{\sigma_{s 2}}{\lambda_{1}}+\eta \frac{\sigma_{1}}{\lambda_{2}}\right)\right] w_{m}}{\left[\sigma_{1} \sigma_{2}+\kappa\left|\mu_{m}\right|\left(\frac{\sigma_{1}}{\lambda_{2}}+\frac{\sigma_{2}}{\lambda_{1}}\right)\right]}
\end{gathered}
$$

So equations Eqs. (177) and (192) through (195) give the exact spectral radius for the recursion Eqs. (114) and (115) for slab geometry with continuous space and discretized 
angle variables.

Now, consider that Eqs. (192) through Eq. (195) are just quadrature rule approximations to integrals over all scattering directions. Indeed, taking the limit of a perfect quadrature, these become integrals over direction. Then in this limit the eigenvalues of the subblocks are

$$
\begin{aligned}
& \Lambda_{1}=\frac{1}{2} \int_{-1}^{1} \frac{\left[\sigma_{s 1} \sigma_{2}+|\mu|\left(\kappa \frac{\sigma_{s 1}}{\lambda_{2}}+\eta \frac{\sigma_{2}}{\lambda_{1}}\right)\right]}{\left[\sigma_{1} \sigma_{2}+\kappa|\mu|\left(\frac{\sigma_{1}}{\lambda_{2}}+\frac{\sigma_{2}}{\lambda_{1}}\right)\right]} d \mu, \\
& \Lambda_{2}=\frac{1}{2} \int_{-1}^{1} \frac{\frac{|\mu|}{\lambda_{2}}\left(\kappa \sigma_{s 2}-\eta \sigma_{2}\right)}{\left[\sigma_{1} \sigma_{2}+\kappa|\mu|\left(\frac{\sigma_{1}}{\lambda_{2}}+\frac{\sigma_{2}}{\lambda_{1}}\right)\right]} d \mu \\
& \Lambda_{3}=\frac{1}{2} \int_{-1}^{1} \frac{\frac{|\mu|}{\lambda_{1}}\left(\kappa \sigma_{s 1}-\eta \sigma_{1}\right)}{\left[\sigma_{1} \sigma_{2}+\kappa|\mu|\left(\frac{\sigma_{1}}{\lambda_{2}}+\frac{\sigma_{2}}{\lambda_{1}}\right)\right]} d \mu \\
& \Lambda_{4}=\frac{1}{2} \int_{-1}^{1} \frac{\left[\sigma_{s 2} \sigma_{1}+|\mu|\left(\kappa \frac{\sigma_{s 2}}{\lambda_{1}}+\eta \frac{\sigma_{1}}{\lambda_{2}}\right)\right]}{\left[\sigma_{1} \sigma_{2}+\kappa|\mu|\left(\frac{\sigma_{1}}{\lambda_{2}}+\frac{\sigma_{2}}{\lambda_{1}}\right)\right]} d \mu .
\end{aligned}
$$

These integrals can be computed explicitly. Define the following quantities

$$
\begin{aligned}
& \alpha_{1}=\lambda_{1} \sigma_{1}, \\
& \alpha_{2}=\lambda_{2} \sigma_{2},
\end{aligned}
$$




$$
\begin{aligned}
& \beta=\frac{\alpha_{1} \alpha_{2}}{\kappa\left(\alpha_{1}+\alpha_{2}\right)}, \\
& g=\beta \ln \left(1+\frac{1}{\beta}\right),
\end{aligned}
$$

where $0 \leq \beta \leq \infty$ and $0 \leq g \leq 1$. Then computing the integrals gives

$$
\begin{gathered}
\Lambda_{1}=\left(\frac{\sigma_{s 1}}{\sigma_{1}}\right) \frac{\left(\alpha_{2} g+\alpha_{1}\right)}{\left(\alpha_{1}+\alpha_{2}\right)}+\left(\frac{\eta}{\kappa}\right) \frac{\alpha_{2}(1-g)}{\left(\alpha_{1}+\alpha_{2}\right)} \\
\Lambda_{2}=\lambda_{1} \sigma_{2}\left(\frac{\sigma_{s 2}}{\sigma_{2}}-\frac{\eta}{\kappa}\right) \frac{(1-g)}{\left(\alpha_{1}+\alpha_{2}\right)}, \\
\Lambda_{3}=\lambda_{2} \sigma_{1}\left(\frac{\sigma_{s 1}}{\sigma_{1}}-\frac{\eta}{\kappa}\right) \frac{(1-g)}{\left(\alpha_{1}+\alpha_{2}\right)}, \\
\Lambda_{4}=\left(\frac{\sigma_{s 2}}{\sigma_{2}}\right) \frac{\left(\alpha_{1} g+\alpha_{2}\right)}{\left(\alpha_{1}+\alpha_{2}\right)}+\left(\frac{\eta}{\kappa}\right) \frac{\alpha_{1}(1-g)}{\left(\alpha_{1}+\alpha_{2}\right)} .
\end{gathered}
$$

Now Eqs. (177), (182) and (204) through (207) give the spectral radius of the iteration equations (114) and (115) for a slab geometry and in the limit of a perfect quadrature, assuming the $\mathrm{k}=0$ mode. One last step is to explicitly find the maximum that the eigenvalues from can attain. Denote the following quantities

$$
\begin{gathered}
c_{i}=\frac{\sigma_{s i}}{\sigma_{i}}, \\
r=\frac{\eta}{\kappa},
\end{gathered}
$$


and

$$
r_{i}=\frac{\sigma_{s i}}{\sigma_{i}}-\frac{\eta}{\kappa}=c_{i}-r
$$

Then if the eigenvalues of $A$ are given by Eq. (173), they become

$$
\Lambda_{ \pm}=\frac{r}{2}+\frac{\left(\alpha_{2} r_{1}+\alpha_{1} r_{2}\right) g+\left(\alpha_{1} c_{1}+\alpha_{2} c_{2}\right) \pm \sqrt{h}}{2\left(\alpha_{1}+\alpha_{2}\right)}
$$

where

$$
h=\left[\left(\alpha_{2} r_{1}-\alpha_{1} r_{2}\right) g+\alpha_{1} r_{1}-\alpha_{2} r_{2}\right]^{2}+4 \alpha_{1} \alpha_{2} r_{1} r_{2}(1-g)^{2}
$$

So the eigenvalues $\Lambda_{ \pm}$as given by Eq. (173) are pseudo-linear functions of $g$. All that remains is to find their largest absolute values.

Lemma 1: $h>0$ for all $0 \leq g \leq 1$.

Proof: Consider Eq. (212). The only parameters which can be negative are the $r_{i}$.

Clearly then $h$ is positive if $r_{1}$ and $r_{2}$ are of the same sign, both negative or both positive.

To see that $h$ is positive also for the case that the $r_{i}$ are of opposite signs, notice that $h$ is a quadratic in $g$. Then $h(g)$ will have one extrema, be it a maximum or a minimum. Label the extremum point $g_{o}$. Solving $\frac{d h}{d g}=0$ for $g$ gives

$$
g_{o}=\frac{r_{1} r_{2}\left(\alpha_{1}^{2}+\alpha_{2}^{2}\right)-\alpha_{1} \alpha_{2}\left(r_{1}^{2}+r_{2}^{2}-4 r_{1} r_{2}\right)}{\left(\alpha_{2} r_{1}+\alpha_{1} r_{2}\right)^{2}}
$$


The point $g_{o}$ is clearly negative when the $r_{i}$ have opposite signs. Since $h$ has only one extrema and that point lies outside the range $0 \leq g \leq 1, h$ must be monotonic in this range when the $r_{i}$ has opposite signs. But $h(0)=\left(\alpha_{1} r_{1}+\alpha_{2} r_{2}\right)^{2}$ and $h(1)=\left(\alpha_{1}+\alpha_{2}\right)^{2}\left(r_{1}-r_{2}\right)^{2}$ are both positive. Therefore $h$ is positive for all $0 \leq g \leq 1$. $Q E D$.

Lemma $2: \frac{\partial h}{\partial g}>0$ for all $0 \leq g \leq 1$ when $r_{1} r_{2}<0$.

Proof: Taking the derivative of $h$ with respect to $g$ gives

$$
\frac{\partial h}{\partial g}=-\alpha_{1} \alpha_{2} r_{1} r_{2}(1-g)+2\left(\alpha_{2} r_{1}-\alpha_{1} r_{2}\right)\left(\alpha_{1} r_{1}-\alpha_{2} r_{2}+g\left(\alpha_{2} r_{1}-\alpha_{1} r_{2}\right)\right) \text {. }
$$

The result is obvious by inspection. $Q E D$.

Lemma 3: The $\Lambda_{ \pm}$are monotonic in $g$ for all $0 \leq g \leq 1$.

Proof: Taking the derivative of $\Lambda_{ \pm}$with respect to $g$ gives

$$
4 \sqrt{h}\left(\alpha_{1}+\alpha_{2}\right) \frac{\partial \Lambda_{ \pm}}{\partial g}=2 \sqrt{h}\left(\alpha_{2} r_{1}+\alpha_{1} r_{2}\right) \pm \frac{\partial h}{\partial g}
$$

And since $4 \sqrt{h}\left(\alpha_{1}+\alpha_{2}\right)>0$ by Lemma 1 ,

$$
\operatorname{sign}\left\{\frac{\partial \Lambda_{ \pm}}{\partial g}\right\}=\operatorname{sign}\left\{2 \sqrt{h}\left(\alpha_{2} r_{1}+\alpha_{1} r_{2}\right) \pm \frac{\partial h}{\partial g}\right\}
$$

Now if 


$$
\left|2 \sqrt{h}\left(\alpha_{2} r_{1}+\alpha_{1} r_{2}\right)\right|>\left|\frac{\partial h}{\partial g}\right|
$$

one can conclude from Eq. (216) that

$$
\operatorname{sign}\left\{\frac{\partial \Lambda_{ \pm}}{\partial g}\right\}=\operatorname{sign}\left\{2 \sqrt{h}\left(\alpha_{2} r_{1}+\alpha_{1} r_{2}\right)\right\}
$$

Likewise if

$$
\left|2 \sqrt{h}\left(\alpha_{2} r_{1}+\alpha_{1} r_{2}\right)\right|<\left|\frac{\partial h}{\partial g}\right|
$$

one can conclude that

$$
\operatorname{sign}\left\{\frac{\partial \Lambda_{ \pm}}{\partial g}\right\}=\operatorname{sign}\left\{ \pm \frac{\partial h}{\partial g}\right\}
$$

To determine if either of these cases is in general true, compute the difference in their squares,

$$
\left(\frac{\partial h}{\partial g}\right)^{2}-\left(2 \sqrt{h}\left(\alpha_{2} r_{1}+\alpha_{1} r_{2}\right)\right)^{2}=-16 \alpha_{1} \alpha_{2} r_{1} r_{2}\left(\alpha_{1}+\alpha_{2}\right)^{2}\left(r_{1}-r_{2}\right)^{2}
$$

The right hand side of Eq. (221) is negative if the $r_{i}$ have the same sign and is positive if the $r_{i}$ have opposite signs. So if the $r_{i}$ have the same sign, then Eq. (218) is true and by Lemma 1 the $\Lambda_{ \pm}$are monotonic for all $0 \leq g \leq 1$. If the $r_{i}$ have opposite signs then Eq. (220) is true and by Lemma 2 the $\Lambda_{ \pm}$are monotonic for all $0 \leq g \leq 1$. $Q E D$.

Because the $\Lambda_{ \pm}$are monotonic in $g$, the maximum values of $\left|\Lambda_{ \pm}\right|$occur either at 
$g=0$ or 1 . Evaluating the $\Lambda_{ \pm}$at $g=0$ and at $g=1$ gives

$$
\left.\Lambda_{ \pm}\right|_{g=0}=\frac{r}{2}+\frac{\left(\alpha_{1} c_{1}+\alpha_{2} c_{2}\right) \pm\left(\alpha_{1} r_{1}+\alpha_{2} r_{2}\right)}{2\left(\alpha_{1}+\alpha_{2}\right)}=r, \frac{\left(\alpha_{1} c_{1}+\alpha_{2} c_{2}\right)}{\left(\alpha_{1}+\alpha_{2}\right)}
$$

and

$$
\left.\Lambda_{ \pm}\right|_{g=1}=\frac{\left(c_{1}+c_{2}\right) \pm\left(c_{1}-c_{2}\right)}{2}=c_{1}, c_{2}
$$

All four of these values are positive. Then using

$$
\frac{\left(\alpha_{1} c_{1}+\alpha_{2} c_{2}\right)}{\left(\alpha_{1}+\alpha_{2}\right)} \leq \max \left[c_{1}, c_{2}\right]
$$

gives the result that

$$
\rho(A)=\max \left[\left|\Lambda_{+}\right|,\left|\Lambda_{-}\right|\right] \leq \max \left[c_{1}, c_{2}, r\right]
$$

If the eigenvalues of the matrix $A$ are given by Eq. (176) then they are linear functions of $g$ and hence their maximums either occur at $g=0$ or 1 . Taking these limits gives

$$
\left.\Lambda_{ \pm}\right|_{g=0}=\frac{\left(\alpha_{1} c_{1}+\alpha_{2} r\right)}{\left(\alpha_{1}+\alpha_{2}\right)}, \frac{\left(\alpha_{1} r+\alpha_{2} c_{2}\right)}{\left(\alpha_{1}+\alpha_{2}\right)}
$$

and

$$
\left.\Lambda_{ \pm}\right|_{g=1}=c_{1}, c_{2}
$$

Then using

$$
\frac{\left(\alpha_{1} r+\alpha_{2} c_{2}\right)}{\left(\alpha_{1}+\alpha_{2}\right)} \leq \max \left[r, c_{2}\right]
$$


and

$$
\frac{\left(\alpha_{1} c_{1}+\alpha_{2} r\right)}{\left(\alpha_{1}+\alpha_{2}\right)} \leq \max \left[r, c_{1}\right]
$$

again recovers the same result Eq. (225) proving Theorem 2. $Q E D$.

The requirement for convergence

$$
\rho(A) \leq 1
$$

then becomes in a 1-D slab geometry

$$
\rho(A) \leq \max \left[c_{1}, c_{2}, r\right] \leq 1 .
$$




\section{APPENDIX B: Discretization Methods}

To generate numerical solutions of the transport equation it is necessary to replace each term in the equation with discrete approximations. The primary terms requiring special attention are the streaming operator $\vec{\Omega} \cdot \nabla \psi$ and the integral over direction $\int_{\text {all } \Omega} \psi d \vec{\Omega}$, where $\vec{\Omega}$ is the direction of photon travel. One common method to discretize the streaming operator is the diamond difference method [17]. A common method to discretize the direction integral is the discrete ordinates method [17].

The diamond difference discretization is a bit different in 1-D slab and 1-D spherical geometries so they are presented separately.

\subsection{Discretizations in a 1-D Slab}

In a 1-D slab geometry the discretization of space and angle can be done independent of each other.

\subsection{Diamond Difference}

Let $z$ denote the spacial axis in a 1-D slab geometry. Then In a 1-D slab geometry the streaming operator becomes

$$
\vec{\Omega} \cdot \nabla \psi=\mu \frac{\partial \psi}{\partial z}
$$

where $\mu$ is the cosine of the angle $\bar{\Omega}$ makes with the $z$ axis. Define $\psi\left(z_{i}\right)=\psi_{i}$. The dia- 
mond difference discretization simply says

$$
\left.\frac{\partial \psi}{\partial z}\right|_{z_{i}} \cong \frac{\Psi_{i+\frac{1}{2}}-\Psi_{i-\frac{1}{2}}}{\left(z_{i+\frac{1}{2}}-z_{i-\frac{1}{2}}\right)}
$$

combined with the auxiliary relation

$$
\psi_{i}=\frac{1}{2}\left(\psi_{1+\frac{1}{2}}+\psi_{1-\frac{1}{2}}\right)
$$

\subsection{Discrete Ordinates}

In the 1-D slab geometry the integral over direction becomes

$$
\int_{\text {all } \Omega} \psi d \vec{\Omega}=\int_{-1}^{1} \psi(z, \mu) d \mu .
$$

The discrete ordinates methods is a replacement of the integral over direction with a quadrature rule approximation. Essentially this means that the possible directions of photon travel are divided into "bins" which act as the ordinates in the quadrature rule. This method is also referred to as the $S_{N}$ method. This in general yields a discrete approximation to the direction integral

$$
\int_{-1}^{1} \psi(z, \mu) d \mu \cong \sum_{n=1}^{N} w_{n} \psi\left(z, \mu_{n}\right)
$$

where the $w_{n}$ are the weights for the chosen quadrature rule and $N$ is the order of the quadrature. 


\subsection{Discretizations in a 1-D Sphere}

In the spherical geometry the discretization of angle and direction are related to each other so they are presented together here.

\subsection{Diamond Difference/Discrete Ordinates}

Let $\rho$ be the radial direction in a 1-D spherical geometry. Let $\mu$ be the cosine of the angle $\vec{\Omega}$ makes with the $\rho$ axis. Then the streaming operator becomes

$$
\vec{\Omega} \cdot \nabla \psi=\mu \frac{\partial \psi}{\partial \rho}+\frac{\left(1-\mu^{2}\right)}{\rho} \frac{\partial \psi}{\partial \mu}
$$

The integral over direction is represented the same as in the 1-D slab geometry, i.e. by Eqs. (235) and (236). But now the streaming operator contains a derivative with respect to angle so that it becomes necessary to discretize the $\frac{\partial \psi}{\partial \mu}$ term on the same ordinates used in Eq. (236). This is done in the following way. The streaming operator is first written in conservation form as

$$
\mu \frac{\partial \psi}{\partial \rho}+\frac{\left(1-\mu^{2}\right)}{\rho} \frac{\partial \psi}{\partial \mu}=\frac{\mu}{\rho^{2}} \frac{\partial}{\partial \rho} \rho^{2} \psi+\frac{1}{\rho} \frac{\partial}{\partial \mu}\left(1-\mu^{2}\right) \psi
$$

Now denote $\psi\left(\mu_{n}, \rho_{i}\right)=\psi_{n, i}$. Then the angular derivative is discretized as

$$
\left.\frac{1}{\rho} \frac{\partial}{\partial \mu}\left(1-\mu^{2}\right) \psi\right|_{\mu_{n}} \cong \frac{2}{\rho w_{n}}\left[\alpha_{n+\frac{1}{2}} \psi_{n+\frac{1}{2}}-\alpha_{n-\frac{1}{2}} \psi_{n-\frac{1}{2}}\right]
$$

The $\alpha_{i}$ are weighting factors which are generated from the quadrature weights according 
to the recursion

$$
\alpha_{n+\frac{1}{2}}=\alpha_{n-\frac{1}{2}}-\mu_{n} w_{n}
$$

The purpose of the $\alpha_{n}$ weights are to guarantee that the streaming term vanishes in the limit of a uniform isotropic flux in an infinite medium. See reference [17] for further discussion.

To discretize the radial derivative the equation is volume averaged over a spherical shell to get

$$
\left.\frac{\mu}{\rho} \frac{\partial}{\partial \rho} \rho^{2} \psi\right|_{\rho_{i} \mu_{n}} \cong \frac{\mu_{n}}{V_{i}}\left[A_{i+\frac{1}{2}} \psi_{n, i+\frac{1}{2}}-A_{i-\frac{1}{2}} \psi_{n, i-\frac{1}{2}}\right]
$$

and

$$
\left.\frac{1}{\rho} \frac{\partial}{\partial \mu}\left(1-\mu^{2}\right) \psi\right|_{\rho_{i} \mu_{n}} \cong \frac{\left(A_{i+\frac{1}{2}}-A_{i-\frac{1}{2}}\right)}{V_{i} w_{n}}\left[\alpha_{n+\frac{1}{2}} \psi_{n+\frac{1}{2}, i}-\alpha_{n-\frac{1}{2}} \psi_{n-\frac{1}{2}, i}\right]
$$

The quantities $A_{i}$ are the surface area of a sphere at radius $i$ and the $V_{i}$ are the volume of a spherical shell from radius $\rho_{i-\frac{1}{2}}$ to $\rho_{i+\frac{1}{2}}$. The Eqs. (240) through (242) are then combined with the auxiliary relations

$$
\psi_{n, i}=\frac{1}{2}\left(\psi_{n, i+\frac{1}{2}}+\psi_{n, i-\frac{1}{2}}\right)
$$

and 


$$
\Psi_{n, i}=\frac{1}{2}\left(\Psi_{n+\frac{1}{2}, i}+\Psi_{n-\frac{1}{2}, i}\right)
$$

to complete the discretization. 


\section{REFERENCES}

1. P. Boisse, Astron. Astrophysics 228, 483 (1990)

2. H. Storzer, J. Stutzki, A. Sternberg, Astron. Astrophysics 310, 592 (1996)

3. A. N. Witt, K. D. Gordon, Astrophys. J. 463, 681 (1996)

4. F. R. Malvagi, R. N. Byrne, R. Somerville, J. Atmos. Sci. 50, 2146

5. G. L. Stephens, P. M. Gabriel, S. C. Tsay, Trans. Theory Statist. Phys. 20, 139 (1991)

6. C. Deutsch and D. Vanderhaegen, JQSRT 44, 163 (1990).

7. N. A. Tahir and K. A. Long, Phys. Fluids 29, 1282 (1986).

8. H. J. Kull, Phys. Rev. A 33, 1957 (1986).

9. C. D. Levermore, G.C. Pomraning, D. L. Sanzo, J. Wong, J. Math. Phys. 27, 2526 (1986).

10. C. D. Levermore, G.C. Pomraning, and J. Wong, J. Math. Phys. 29, 995 (1988).

11. G.C. Pomraning, Linear Kinetic Theory and Particle Transport in Stochastic Mixtures, World Scientific, Singapore (1991).

12. D. Vanderhaegen, JQSRT 36, 557 (1986).

13. D. Vanderhaegen and C. Deutsch, J. Statist. Phys. 54, 331 (1989).

14. M. L. Adams, E. W. Larsen, and G.C. Pomraning, JQSRT 42, 253 (1989).

15. Richard Sanchez, J. Math. Phys. 30, 2498 (1989).

16. Bingjing Su and G.C. Pomraning, JQSRT 51, 893 (1994).

17. E. E. Lewis and W. F. Miller Jr., Neutron Transport, American Nuclear Society Inc., 
Lagrange Park, Illinois (1993).

18. D. R. Cox, Renewal Theory, Methuen \& Co. LTD, London (1970)

19. S. Audic, H. Frisch, JQSRT 50, 127(1993).

20. A. Brissaud, U. Frisch, J. Math. Phys. 15, 524 (1974).

21. J. A. Viecelli, Applied Optics 35, (1996)

22. E. W. Larsen, Nuclear Sci. and Eng. 82, 47 (1982)

23. C. Lin, J. W. Harbaugh, Graphic Display of two and three dimensional Markov Computer Models in Geology, Van Nostrand Reinhold, New York (1984) 